\title{
PRIVACY ATTITUDES AND BEHAVIOURS OF AUTISTIC AND NON-AUTISTIC TEENAGERS ON SOCIAL NETWORKING SITES
}

\author{
by \\ Jessica Nicole Rocheleau
}

A thesis submitted to

the Faculty of Graduate and Postdoctoral Affairs

in partial fulfillment of

the requirements for the degree of

MASTER OF ARTS

Human Computer Interaction

at

CARLETON UNIVERSITY

Ottawa, Ontario

August, 2019

(C) Copyright by Jessica Nicole Rocheleau, 2019 


\begin{abstract}
Researchers postulate that autistic teenagers are more vulnerable to privacy threats on social networking sites (SNS) than the general population. However, there are no studies comparing these users' privacy concerns and protective strategies online with those reported by non-autistic teenagers. Furthermore, researchers have yet to identify possible explanations for autistic teenagers' exceptional risk of online harms. To address these research gaps, we conducted semi-structured interviews with 12 autistic and 16 non-autistic teenagers assessing their privacy attitudes and behaviours on SNS, and factors affecting their privacy. We used videos demonstrating relevant SNS scenarios as prompts to engage participants in conversation. Our thematic analyses demonstrated that autistic participants were more averse to taking risks on SNS than non-autistic participants. Yet, several personal, social, contextual, and SNS design factors made autistic participants exceptionally vulnerable to cyberbullying and social exclusion online. We provide recommendations for making SNS safer and more inclusive for autistic teenagers.
\end{abstract}




\section{Acknowledgements}

I have never been more excited to fill a blank page. There are so many people I want to thank for making my time as a Master's student the most productive, exciting and fulfilling years of my life so far. First and foremost, I am infinitely grateful to my incredible supervisor, Dr. Sonia Chiasson. Thank you, Sonia, for the endless patience, helpful feedback, and amazing opportunities you offered me over the past two years. You are the best supervisor any student can ask for, and an inspiration to me. I am so lucky that I get to continue being mentored by you throughout my PhD.

Thank you to my colleagues in the CHORUS lab for helping me to prepare for my thesis defense. I am also grateful to my committee members, Dr. Kasia Muldner and Dr. Elizabeth Stobert, for their insightful questions and feedback on my research.

It was a privilege to collaborate with Dr. Virginie Cobigo and Dr. Hajer Chalghoumi on various projects throughout my Master's degree. These two amazing researchers opened my eyes to the many barriers that people with cognitive disabilities face with accessing and using information technologies, and inspired me to highlight the voices of this population through my inclusive research. Thank you, Virginie and Hajer, for helping me to develop my passion and expertise in my area of research.

Thank you to my loving family and friends for your encouragement and support. I want to give a special shout-out to my brilliant, talented and kind sister, Sarah, for being my "biggest fan" (her words, not mine). Thank you, Sarah, for your somewhat hostile pep talks; for trying to understand my incoherent rambles about my research; for distracting me when I was stressed out; and for helping me with the videos for my study. I'll keep you around, I guess - you're worth more than 10 nickles to me. ;)

I also owe a debt of gratitude to the autism organizations and groups who shared my study details. This project would not have been possible without their support.

Finally, to my participants: thank you for investing the time and effort to open up about your experiences with me. It was a pleasure speaking to every one of you, and I hope that this report represents you well. This thesis is dedicated to you. 


\section{Table of Contents}

Abstract

Acknowledgements $\quad$ iii

List of Tables $\quad$ vii

List of Figures viii

Chapter 1 Introduction 1

1.1 Motivation ....................... . . . 1

1.2 Research Question .................... . . . 2

1.3 Contribution . . . . . . . . . . . . . . . . 2

1.4 Thesis Outline . . . . . . . . . . . . . . . . . 3

$\begin{array}{lll}\text { Chapter } 2 & \text { Background } & 4\end{array}$

2.1 Autism . . . . . . . . . . . . . . . . . . 4

2.1.1 Medical Perspective . . . . . . . . . . . . . . . . 4

2.1.2 Social Perspective . . . . . . . . . . . . . 5

2.2 Social Networking Sites . . . . . . . . . . . . . . . . 6

2.3 Teenagers' Social Networking Site Use . . . . . . . . . . . . . . 6

2.3.1 Benefits ..................... 7

2.3.2 Risks ....................... 8

2.4 Privacy Attitudes . . . . . . . . . . . . . . . 9 9

2.5 Privacy Behaviours . . . . . . . . . . . . . . . . 11

2.6 Factors Affecting Privacy Attitudes and Behaviours . . . . . . . . . 13

2.6.1 Age .............................. 13

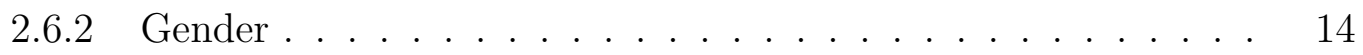

2.6.3 Disposition . . . . . . . . . . . . . . . 15

2.6.4 Frequency of Active Social Networking Site Use . . . . . . . . 16 
2.6.5 Previous Experience with Privacy Violations . . . . . . . . . . 16

2.6.6 Parental Mediation . . . . . . . . . . . . . . . . . . 17

2.6.7 Online Privacy Educators . . . . . . . . . . . . . . . 18

2.6 .8 Peer Influence . . . . . . . . . . . . . . . . . . . . . . . 19

2.6.9 Usability of Social Networking Sites . . . . . . . . . . . . . 19

2.7 Current Study . . . . . . . . . . . . . . . . . . . . 21

$\begin{array}{lll}\text { Chapter } 3 & \text { Methodology } & 24\end{array}$

3.1 Participants . . . . . . . . . . . . . . . . . . . . 24

3.1.1 Recruitment . . . . . . . . . . . . . . . 24

3.1 .2 Demographics ....................... 25

3.2 Materials . . . . . . . . . . . . . . . . . . . 27

3.2.1 Interview Guide . . . . . . . . . . . . . . . . . . . 27

3.2.2 Scenario Video Clips . . . . . . . . . . . . . . . . . . . . 28

3.3 Procedure . . . . . . . . . . . . . . . . . . . . 31

3.4 Qualitative Data Analysis . . . . . . . . . . . . . . 32

Chapter 4 Results 33

4.1 Privacy Attitudes . . . . . . . . . . . . . . . . . 33

4.1.1 Knowledge and Awareness of Privacy Threats . . . . . . . . . 33

4.1.2 Privacy Concerns . . . . . . . . . . . . . . . . . . 35

4.1.3 Balancing Privacy and Self-Disclosure . . . . . . . . . . . . 39

4.2 Privacy Behaviours . . . . . . . . . . . . . . . . 41

4.2.1 Deciding Who to Trust . . . . . . . . . . . . . 41

4.2.2 Managing Access . . . . . . . . . . . . . . . . . . 43

4.2 .3 Negotiating Consent . . . . . . . . . . . . . . . . . 46

4.2.4 Filtering Out Personal Information . . . . . . . . . . . . . 47

4.3 Factors Affecting Privacy Attitudes and Behaviours . . . . . . . . . 48

4.3.1 Personal Factors . . . . . . . . . . . . . . 50

4.3.2 Social Factors . . . . . . . . . . . . . . 58

4.3.3 Contextual Factors . . . . . . . . . . . . . . . . . 62 
4.3.4 Social Networking Site Design Factors . . . . . . . . . . . 63

4.4 Summary . . . . . . . . . . . . . . . . . . 72

4.4.1 Privacy Attitudes . . . . . . . . . . . . . . . 72

4.4.2 Privacy Behaviours ................. . . 72

4.4.3 Privacy-Related Factors . . . . . . . . . . . . . . . . . 73

$\begin{array}{lll}\text { Chapter } 5 & \text { Discussion and Conclusion } & 77\end{array}$

5.1 Lessons Learned . . . . . . . . . . . . . . . . . . . . . . . . 77

5.1.1 Autistic Users' Vulnerability to Online Risks: Exposing Misconceptions through Inclusive Research . . . . . . . . . . . 78

5.1.2 Disparities in the Benefits and Risks of Online Social Networking 80

5.1.3 Balancing Online Privacy and Sociability . . . . . . . . . . . 83

5.1.4 Inter-relatedness of Accessibility, Privacy and Autonomy . . . 84

5.1.5 Accessibility is for Everyone . . . . . . . . . . . . . . . 85

5.2 Recommendations . . . . . . . . . . . . . . . . 85

5.2.1 Addressing Personal Factors . . . . . . . . . . . . . . 86

5.2.2 Addressing Social Factors . . . . . . . . . . . . 88

5.2.3 Addressing Contextual Factors . . . . . . . . . . . . . 89

5.2.4 Addressing Social Networking Site Design Factors . . . . . . . 90

5.3 Limitations and Future Work . . . . . . . . . . . . . . . . . . 90

5.4 Conclusion . . . . . . . . . . . . . . . . . . . . . . . 91

$\begin{array}{ll}\text { Bibliography } & 93\end{array}$

$\begin{array}{lll}\text { Appendix A Recruitment Materials } & 106\end{array}$

$\begin{array}{lll}\text { Appendix B Consent Form } & 112\end{array}$

$\begin{array}{lll}\text { Appendix C Interview Guide } & 117\end{array}$ 


\section{List of Tables}

2.1 Summary of SNS use, privacy attitudes, and privacy behaviours 22

2.2 Summary of factors affecting privacy attitudes and behaviours .

3.1 Summary of participant demographics . . . . . . . . . 25

3.2 Detailed participant demographics and preferred SNS . . . . 26

4.1 Definition of factors affecting privacy attitudes and behaviours $\quad 49$

4.2 Summary of findings: privacy attitudes, privacy behaviours, personal factors . . . . . . . . . . . . . . . . 75

4.3 Summary of findings: social factors, contextual factors, SNS design factors ................... . . 76 


\section{List of Figures}

3.1 Screenshots representing Scenarios 1-3 from Instagram. . . . . 29

a Scenario 1 - general self-disclosure . . . . . . . . . . . . . 29

b Scenario 2 - self-disclosing to strangers . . . . . . . . . . . 29

c Scenario 3 - non-consensual disclosure by others . . . . . . . 29

$3.2 \quad$ Screenshots representing Scenarios 4-5 from Instagram. . . . . 30

a Scenario 4 - using privacy settings . . . . . . . . . . 30

b Scenario 5 - other privacy protection strategies . . . . . . 30 


\section{Chapter 1}

\section{Introduction}

\subsection{Motivation}

Social networking sites (SNS) play a crucial role in teenagers' lives. Today, nearly all teenagers use these platforms to build and maintain relationships, express themselves, and explore their identity online $[10,64,122]$. Although all teenagers can benefit from using SNS, these platforms may be especially useful for autistic teenagers.

Autistic teenagers tend to be lonely and socially isolated in real life, due to their extreme difficulties with knowing how to act in social situations. Conceptually, the anonymity of SNS can provide a comfortable environment for autistic teenagers to connect with others, make friends, and thus improve their social-emotional well-being $[12,20,89]$. However, research suggests that these users are more vulnerable to privacy and safety threats on SNS (e.g., cyberbullying, sexual harassment) than the general population $[39,48]$, despite the fact that most members of this population use these platforms less frequently than their typically developing peers $[68,70]$.

To date, little is known about autistic teenagers' awareness of and experiences with privacy risks on SNS, as well as their strategies for preventing and coping with online threats. Although researchers have investigated caregivers' and experts' perspectives on this matter, there is an absence of studies exploring autistic users' point-of-view. Additionally, the vast majority of studies speculating on autistic teenagers' extreme vulnerability to online risks do not directly compare these users' negative experiences on SNS with those reported by non-autistic teenagers. These limitations in previous research related to autistic teenagers' privacy and safety online may be leading to misconceptions about this population's vulnerability to online threats. If so, these misconceptions must be exposed in order to properly address these users' needs.

Moreover, there is a paucity of research identifying factors that may be affecting autistic teenagers' privacy-related thoughts and protective strategies on SNS. Indeed, 
most studies attempting to explain these users' exceptional vulnerability to online threats mainly focus on the symptoms of autism spectrum disorder, with far less consideration for external causes. Consequently, there is a lack of guidance and initiative to address environmental barriers that may be preventing autistic teenagers from developing effective strategies to protect their privacy in online social contexts. There is a need to gain a more holistic understanding of the facilitators and barriers to autistic teenagers' privacy protection to improve these users' safety online.

\subsection{Research Question}

The goals of this study are to compare the privacy attitudes and behaviours of autistic and non-autistic teenagers on SNS, and identify factors affecting these users' privacy and safety online. More specifically, we will examine the following research questions: RQ1: What are autistic teenagers' thoughts and feelings about their privacy on SNS, and how do they protect their online privacy?;

RQ2: What are the main factors affecting autistic teenagers' privacy attitudes and behaviors on SNS?; and

RQ3: How do these privacy-related attitudes, behaviours, and factors compare with those reported by non-autistic teenagers?

\subsection{Contribution}

The main contributions of our study are as follows:

1. To our knowledge, this is the first study to examine autistic teenagers' privacy attitudes and behaviours on SNS from the users' own perspectives. It is also the first study to compare autistic and non-autistic teenagers' vulnerability to online risks, and identify key differences in factors affecting these populations' privacy on SNS. Therefore, our research provides novel insight into autistic teenagers' unique experiences with preventing online harm. In this thesis, we offer a detailed report of empirical findings regarding autistic and non-autistic teenagers' privacy attitudes and behaviours on SNS, and factors affecting their online privacy and safety. Our findings expose the common misconception that 
autistic teenagers are especially vulnerability to social privacy risks on SNS. We also identify key personal, social, contextual, and SNS design factors that disparately impact these users' privacy and active engagement in online social activities.

2. We demonstrate an effective methodology for an inclusive user study with autistic and non-autistic teenagers. In particular, our study involved a semistructured interview that was guided by relevant SNS scenario videos as prompts to engage participants in conversation.

3. We relate our findings to existing knowledge in our research area, and provide recommendations to make SNS safer and more inclusive for autistic teenagers. Our research highlights the need for greater initiative by the Web community to prioritize cognitive accessibility in their design practices. We outline the benefits of this cultural shift for improving both autistic and non-autistic teenagers' experiences using these platforms. Moreover, this thesis offers a discussion about the ethical, developmental, and psychological consequences related to the inaccessibility of SNS and restrictive mediation strategies used to shield autistic teenagers from online harms. Ultimately, this study aims to provide knowledge and practical recommendations to balance the risks and benefits associated with autistic teenagers' SNS use.

\subsection{Thesis Outline}

In Chapter 2, we provide an overview of the current literature related to our research topic. Chapter 3 describes the methodology of our study, which is then followed by a detailed report of our findings in Chapter 4. Finally, in Chapter 5, we discuss the implications of our findings, limitations of our study, and areas for future research. 


\section{Chapter 2}

\section{Background}

\section{$2.1 \quad$ Autism}

Like all disabilities, autism can be defined from two different viewpoints [52]: (1) the medical model, which describes disability as an "individual problem tied to the functional limitations of the bodies of people with impairments" [112], or, (2) the social model, which emphasizes the role of social and political infrastructures in disabling people who deviate from the norm [41]. In the following sections, we describe autism from medical and social perspectives, and identify our approach to defining the characteristics and needs of autistic people in our study.

\subsubsection{Medical Perspective}

From a medical standpoint, autism is a neurodevelopmental disorder that is mainly characterized by impairments in social functioning [8]. It is estimated that 1 in 59 people are autistic [8], with males four times more likely to be diagnosed with autism spectrum disorder than females [34]. The most recent edition of the Diagnostic and Statistical Manual of Mental Disorders (DSM-5, [5]) identifies two groups of symptoms inherent to autism spectrum disorder:

1. Persistent difficulties with social communication and interaction, such as deficits in social-emotional reciprocity (e.g., turn-taking in conversations, initiating social interactions, sharing feelings); non-verbal communication (e.g., using gestures, body language and eye contact); and in developing, maintaining, and understanding relationships (e.g., knowing how to adjust behaviours for different social contexts, making friends).

2. Restricted and repetitive patterns of behaviour, interests, or activities, such as repetitive movements, interactions with objects, or speech (e.g., lining up objects, echolalia); insistence on sameness and adherence to routines 
(e.g., extreme distress to minor changes, rigid thought patterns); highly fixated interests (e.g., abnormally strong attachment to objects); and hyper- or hyporeactivity to sensory stimuli (e.g., aversion to specific sounds or textures). To be diagnosed with autism spectrum disorder, the person must exhibit symptoms in these two domains at an early age. Their symptoms must also cause significant impairments in aspects of their daily functioning.

Autism frequently co-occurs with intellectual disability (intellectual developmental disorder) [5]. Indeed, nearly 70 percent of autistic people have intellectual disabilities, leading to difficulties with reading, processing and recalling information [57]. It is also often comorbid with other mental illnesses, such as social anxiety, depression, and attention deficit hyperactivity disorder (ADHD) $[5,55]$.

\subsubsection{Social Perspective}

In recent years, there has been a shift in the scientific community from labelling autism as a psychiatric disorder to appreciating it as natural human variation in "brainwiring", known as neurodiversity [44]. Advocates of neurodiversity acknowledge the symptoms (or traits) of autism outlined in the DSM-5 [5]. However, they argue that autistic people's impairments in functioning are mainly the product of their discrimination and marginalization by society [44]. Historically, autistic people have been treated as less than human by medical professionals and other authority figures. Indeed, when describing autistic children, Ivar Lovaas, the father of a common therapy for autism called applied behaviour analysis, said: "you have a person in the physical sense - they have hair, a nose and a mouth — but they are not people in the psychological sense" [124]. Thankfully, society has come a long way in terms of becoming more accepting of autistic people. However, this population still faces a substantial amount of social exclusion, including in technology development [24].

In this study, we uphold the social model of disability when identifying challenges that autistic teenagers encounter with protecting their privacy on SNS. We do this by emphasizing external factors that may be affecting these users' privacy attitudes and behaviours, instead of focusing solely on their limitations. We view autism not as a disorder that needs to be cured, but as a community of people with special abilities 
and needs, whose unique perspectives can bring tremendous value to improving SNS' design. Our commitment to respecting the dignity and preferences of the autism community is reflected in our decision not to identify our autistic participants using labels that define these users based on their purported level of functioning (e.g., "highfunctioning" autism). These terms are out-dated anyways, especially given that the American Psychiatric Association removed former sub-classifications of autism (e.g., Asperger syndrome) in the DSM-5 [36].

\subsection{Social Networking Sites}

Boyd and Ellison [15] define SNS as "web-based services that allow individuals to (1) construct a public or semi-public profile within a bounded system, (2) articulate a list of other users with whom they share a connection, and (3) view and traverse their list of connections and those made by others within the system". Essentially, SNS (e.g., Instagram, Facebook, Twitter) are designed to facilitate communication and to connect friends, family and people with similar interests, backgrounds or experiences. Over the past decade, hundreds of SNS have been developed and integrated into the daily routines of billions of people across the world [15]. These sites provide users a convenient platform to share their thoughts, opinions and knowledge with others anytime and anywhere [114].

\subsection{Teenagers' Social Networking Site Use}

SNS use is practically ubiquitous among teenagers. Indeed, it has been estimated that 95 percent of people between 13 and 17 years of age in the U.S. have access to a smartphone, with 45 percent of these users reporting to be online "almost constantly" [3]. Currently, the most popular SNS among teenagers are YouTube, Instagram, Snapchat, Facebook, and Twitter [3].

Teenagers primarily use SNS to communicate, provide feedback (i.e., likes, comments), and stay in touch with their offline friends and significant others [33,118], as well as to widen their social circle by connecting with new people who have similar interests $[18,30,107]$. SNS also provide teenagers a platform to share their thoughts, 
feelings, and personal experiences publicly or semi-publicly by posting status updates and photos on their profile $[2,107]$. Moreover, SNS serve as a main source of news, information and entertainment for people in this age group [114,118].

To date, there are few studies examining autistic teenagers' SNS use. However, some research suggests that these users do not adopt SNS as readily as their typically developing peers $[68,70]$. Kuo et al. [54] examined autistic teenagers' media use. The researchers found that only a few of their participants used computers for social communication (e.g., social networking, instant messaging); yet, those who did spent a relatively long time engaging in online social interactions, suggesting that these users may rely on computer-mediated communication to meet their social needs. Similarly, Gillespie-Lynch et al. [38] compared autistic and non-autistic adults' preferred functions in computer-mediated communication. They found that autistic participants used the Internet more to meet new people and for information (e.g., about hobbies, dating) than non-autistic participants; however, autistic participants liked using SNS less than the general population. Furthermore, Bahiss et al. [7] found that autistic teenagers prefer to interact with others online as a part of a group activity, such as multiplayer gaming, rather than through SNS. Nevertheless, a recent study reports that these users rarely take advantage of the multiplayer option on videogames, often choosing to play alone [80].

There are many risks and benefits associated with autistic and non-autistic teenagers' SNS use. We provide an overview of these outcomes in the sections below.

\subsubsection{Benefits}

Several studies demonstrate the benefits of SNS use on teenagers' growth and wellbeing $[2,84,114]$. From a developmental perspective, adolescence is a critical life stage for identity development [107]. SNS provide teenagers a platform to explore and establish who they are, including their interests, social status, sexuality and affiliations $[2,30,84,107,114,128]$. Teenagers' SNS use also often coincides with increased social support, bonding and sense of belonging, as well as improved self-esteem $[2,30,84]$.

Although SNS can benefit all teenagers, they may be especially useful for those with autism. Several researchers attest to the potential of SNS to enhance these 
users' social and psychological well-being [20,70,89,98]. Autistic teenagers have the same desire to build strong relationships and to belong as their typically developing peers [49]. However, members of this population are especially susceptible to loneliness and social isolation because of their difficulties with social communication and social anxiety $[31,49,65]$. The anonymity and reduced social cues of SNS offer these users a relatively comfortable communication environment, where they can exert more control over their self-presentation and better mitigate over-stimulation during social interactions than in the real world $[12,20,89]$. Autistic people who use SNS to establish or maintain relationships report greater social satisfaction than those who do not [54], and moderate social media use by this population is associated with positive mental health outcomes, namely increased happiness [126]. Furthermore, Kuder and Xin [53] demonstrate the potential for SNS to serve as a tool for helping autistic people to learn and practice social skills (e.g., turn-taking in conversations) that they can apply in both online and offline communication contexts.

\subsubsection{Risks}

Despite the many benefits of SNS use, there are several privacy, safety and mental health risks associated with participating in online social activities. Teenagers are known for sharing an extensive amount of photos, videos and comments online, some of which may be sensitive or controversial [129]. In turn, these users are at high risk of developing a controversial digital footprint that may jeopardize their reputation and future academic or employment opportunities $[2,32,114]$. Considering that teenagers are usually unaware of social media companies' data sharing practices [1,79], they may also be strongly susceptible to non-consensual use of their data for targeted commercial advertising. Moreover, it is becoming common for teenagers to receive unwanted attention from strangers online $[27,61,114]$. In worst-case scenarios, this attention may come from online predators (e.g., stalkers, pedophiles, hackers, scammers) who may solicit and misuse their victims' personal details, causing them financial, physical, psychological and/or social harm [114]. There are also growing concerns that 
teenagers may become unwillingly exposed to inappropriate content from acquaintances or strangers during online encounters [27]. Furthermore, some teenagers experience bullying on SNS by strangers or peers $[4,100]$. Cyberbullying can take many different forms, including hurtful comments, ostracism, and non-consensual disclosure of sensitive personal information $[2,9]$. If left unaddressed, it can have devastating consequences on teenagers' lives, sometimes triggering depression, poor academic performance, social withdrawal, and suicidal ideation [4,100]. Furthermore, experimental studies show that passive SNS use may be detrimental to users' subjective well-being, because it evokes unrealistic social comparisons and envy [120,121].

Some research suggests that autistic teenagers may be especially vulnerable to certain threats on SNS. For instance, studies show that autistic people are disproportionately involved in cyberbullying $[20,48,50,88,130]$. Kowalski and Fedina [50] investigated the prevalence of cyberbullying among autistic youth. They found that over 20 percent of participants had been victims of cyberbullying within the past two months: nearly double the 11 percent prevalence rate among youth without spe-

cial needs [51]. In turn, autistic youth are more at risk of experiencing negative mental health outcomes from using SNS than their typically developing peers. Furthermore, those with intellectual disabilities may be particularly vulnerable to sexual and financial exploitation online, due to their limitations in logical reasoning and assertiveness $[19,22,75]$. On the other hand, autistic users may be more resilient to social engineering attacks than the general population because of their high attention to factual information and details [73].

\subsection{Privacy Attitudes}

In the media, teenagers are often portrayed as being apathetic to privacy risks on SNS. However, research suggests that nearly all teenagers value their privacy online [101]. Indeed, Agosto and Abbas [1] found that only 2 of the 98 teenagers in their study expressed no concerns about posting personal information online. In general, teenagers are mainly wary of social privacy threats (i.e., "violations by other users of the site" [135]) online. Most teenagers are concerned about exposing their personal content to unintended audiences, especially authority figures (e.g., parents, 
employers) and "creepy" strangers who they imagine to be lurking online $[47,133]$. Many also worry about receiving hurtful comments on their posts [1]. A smaller subset of teenagers are wary of non-consensual disclosure, identity fraud, computer security threats (e.g., hacking), and physical safety risks (e.g., sexual assault) related to online social networking $[1,18]$.

Although most teenagers value their privacy on SNS, they widely vary in the degree to which they are willing to trade their personal information for perceived social benefits. Soffer and Cohen [101] found that teenagers can be clustered into four groups with regards to their privacy perceptions on SNS:

1. Teenagers in the first group (accounting for 20 percent of Soffer and Cohen's [101] sample) have pessimistic attitudes towards SNS. They think that it is not important to develop an authentic profile, and do not feel the need to share personal information online.

2. Those in the second group (23 percent) place a high degree of importance on maintaining control over their personal information on SNS through security and privacy tools. They believe that their privacy is more valuable than developing an authentic profile.

3. Members of the third group (28 percent) think that it is important to have an authentic profile, while also taking measures to protect one's privacy. They share personal information on SNS, and use privacy and security tools to manage access to their content.

4. Teenagers in the fourth group (29 percent) have the most liberal privacy attitudes. They believe that it is very important to have an authentic profile, and share their personal information without using privacy and security tools.

Overall, more than half of the participants in Soffer and Cohen's [101] sample were concerned about their privacy and security on SNS, but also prioritized self-disclosure. This finding is consistent with other research demonstrating that teenagers are usually attentive to both the costs and benefits of social media use, and attempt to strike the right balance based on their personal preferences [18, 26, 123].

To date, little is known about autistic teenagers' privacy attitudes on SNS. According to caregivers and professionals, autistic people are often naive to privacy and safety 
threats both online and in real life, mainly due to their intellectual disabilities $[48,72]$. Nevertheless, some studies suggest that people with intellectual disabilities are often highly concerned about the online privacy and safety threats that they are aware of and that are meaningful to them. Molin et al. [72] examined parents' and teachers' views on young people with intellectual disabilities' Internet use. Teachers reported that a few of their students with special needs were "too cautious" about meeting people on SNS. The teachers said that these students believed that it was "too dangerous and adventurous" to attempt to make friends online. Moreover, Kydland et al. [56] observed that the Flikr users with intellectual disabilities in their study were worried about their personal photos being publicly available to others. Therefore, it is more than likely that autistic teenagers - including those with intellectual disabilities - do in fact have privacy concerns related to social media use. The precise nature of these concerns has yet to be explored through empirical research.

\subsection{Privacy Behaviours}

Risk-taking is a central feature of typical adolescent development [108]. During puberty, major changes occur in the brain's socio-emotional system, contributing to increased reward-seeking, particularly in social contexts $[87,109]$. In most cases, teenagers' propensity to engage in risky behaviours extends to their activities in the virtual world. Although teenagers value their privacy and are usually aware of at least some of the potential negative outcomes of posting risky content (e.g., photos of partying, alcohol use, or nudity) or meeting people on SNS, many engage in these relatively high-risk behaviours to satisfy their social and emotional impulses $[27,76,118]$.

Nevertheless, teenagers - like all social media users - require a certain level of privacy to feel comfortable self-disclosing online [16]. Teenagers engage in a variety of privacy-protective behaviours on SNS. Johnson et al. [47] investigated teenagers' privacy decisions about photo sharing on social media. They identified six key strategies that teenagers use to control who can access and distribute their content:

1. Selecting audiences. Teenagers are highly conscientious about managing who can view their photos, due to both privacy and self-presentation concerns. They manage their audience by using privacy settings or (more commonly) not 
posting photos that they do not want "the whole world" to see.

2. Selecting platforms. Instead of keeping photos completely private, most teenagers strategically choose which platform to post particular photos. Their choice of platform usually depends on the affordances and atmosphere of the site. For instance, teenagers often reserve their goofy photos for Snapchat, knowing that posts will disappear. Their Instagram posts, on the other hand, are much more carefully curated, due to the relative permanency of profile photos.

3. Selecting accounts. Many teenagers manage their online audience by creating multiple accounts that serve different purposes. For example, Instagram users may have a public main account with professional-looking photos, and a private "spam" account to share more candid content with close friends.

4. Limiting screenshots. Teenagers are aware that users are notified when someone takes a screenshot of disappearing photos. For teenagers, this feature serves as a social signal that the disappearing photo should not be screenshotted.

5. Negotiating consent. Most teenagers trust their friends to ask before posting a photo of them. When actively seeking consent to post a photo of their friends, teenagers are more likely to ask "Which one should I share?" rather than "Should I share it?", selecting the photo in which everyone looks best. However, in most cases, teenagers do not seek their friends' explicit consent before posting a photo of them. Instead, they rely on their intuition (i.e., imagining their friends' reaction) to determine whether it is appropriate to share the photo.

6. Deleting photos. Rather than actively seeking their friends' approval to post a photo of them, teenagers often rely on "retroactive consent" — that is, asking their friends to delete posted photos that they do not want to be shared.

Researchers have yet to examine autistic teenagers' privacy behaviours on SNS. However, some research suggests that these users - especially those with intellectual disabilities - have more difficulty protecting their privacy online than the general population. Several studies $[19,20,43]$ describe situations in which users with intellectual disabilities engage in risky personal information disclosure on SNS without understanding the potential negative consequences of their actions or knowing how 
to protect themselves against threats. For instance, adults with intellectual disability in Holmes and O'Loughlin's [43] study publicly posted their contact information on their Facebook profile, and shared their login credentials with their friends. These disclosures resulted in privacy violations, such as unwanted messages, sexual exploitation and unauthorized personal information disclosure by others. Similarly, Burke et al. [20] explored autistic adults' social use of computer-mediated communication. Although their study did not explicitly examine these users' privacy behaviours on SNS, the researchers mentioned that a few participants encountered two main challenges related to protecting their privacy during online social interactions: (1) knowing who to trust, and (2) knowing how much personal information is appropriate to disclose to others on the Internet.

\subsection{Factors Affecting Privacy Attitudes and Behaviours}

Teenagers' privacy attitudes and behaviours are affected by numerous personal and external factors. We provide on overview of some of the main factors highlighted in previous research in the sections below.

\subsubsection{Age}

Research shows that teenagers' privacy concerns and protective behaviours decrease with age $[18,32,42,67,118]$. Generally, older teenagers report greater self-efficacy to identify and mitigate threats on SNS than younger teenagers, partly due to their increased experience using these platforms $[1,67]$. Whereas younger teenagers usually comply with their parents' privacy and safety guidelines, older teenagers follow their own intuition about how to navigate complex social situations and manage their personal information on SNS $[117,123]$. By consequence, older teenagers are more likely to engage in risky behaviours (e.g., talking to strangers, sexting, publicly posting a substantial amount of personal information) and experience privacy violations on SNS than younger users $[18,61,76,118]$.

The extent to which these findings apply to autistic teenagers' privacy attitudes

and behaviours is unknown. From a clinical standpoint, autistic people tend to 
demonstrate an increase in comorbid psychiatric disorders (e.g., depression, anxiety, ADHD) as they transition from childhood to adolescence [58]. Teenagers who suffer from mental illnesses are often more at risk of experiencing privacy violations on SNS than the general population [84]. Therefore, older autistic teenagers may be more vulnerable to privacy threats on SNS than their younger counterparts. On the other hand, longitudinal research shows that autistic people tend to demonstrate better social skills and greater interest in social relationships during adolescence than in childhood [58]. Thus, it is possible that older autistic teenagers are more sociable on SNS than younger autistic users, and may therefore be at lower risk of cyberbullying than their younger counterparts.

\subsubsection{Gender}

Gender is another key predictor of teenagers' privacy-related thoughts and behaviours on SNS $[23,76,100,133]$. Several studies suggest that female teenagers typically have higher privacy concerns and engage in more privacy-protective behaviours (e.g., using privacy settings) than males $[23,67,133]$. These gender differences may be partly due to the higher prevalence of sexual solicitation of females on SNS compared to males [18]. Females also tend to post more personal content (including risky photos) on SNS than males $[30,33,61,118]$. On the other hand, male social media users tend to consume more violent content and engage in more aggressive behaviours online than females $[61,133]$.

Researchers have yet to examine the influence of gender on autistic people's privacy attitudes and behaviours on SNS. However, some studies suggest that gender is not a main factor affecting autistic people's social media use. For instance, Mazurek [69] found that there were no significant gender differences in how frequently autistic adults used SNS nor their friendship quality and quantity online. Similarly, MacMullin et al. [68] found that autistic youth, regardless of their gender, use electronics for fewer social activities than their typically developing counterparts. 


\subsubsection{Disposition}

Teenagers' privacy attitudes and behaviours widely vary depending on their personal disposition (e.g., personality, mental health). Several researchers have examined the influence of the Big Five personality traits (i.e., conscientiousness, agreeableness, neuroticism, openness, extroversion [92]) on young people's SNS use and, in turn, their online privacy. Teenagers who are highly extroverted and open to new experiences tend to use SNS more actively than users lower in these traits [14,81]. Highly neurotic individuals frequently use SNS, but tend to conceal aspects of their offline identity due to self-presentation concerns [29,91]. Exceptionally agreeable people are unlikely to be involved cyberbullying [116]. Furthermore, highly conscientious and agreeable users tend to have strong information privacy concerns on SNS [78].

In addition to their personality, teenagers' mental health status also impacts their privacy attitudes and behaviours on SNS. Radovic et al. [84] examined clinically depressed teenagers' positive and negative uses of SNS. They found that depressed teenagers tend to over-share personal details and post regretful content on SNS when stressed in order to cope with their emotions and receive social support. These maladaptive and risky behaviours often result in several negative consequences, such as cyberbullying and poor mood. Other studies show that people with clinical levels of social anxiety heavily rely on computer-mediated communication to compensate for their offline social impairments, making them especially susceptible to problematic Internet use $[59,104]$. At the same time, these users are cautious about their personal information disclosures on SNS - not necessarily because online privacy concerns, but - due to fear of negative evaluation [93].

Research shows that autistic people tend to score high in neuroticism and low in extroversion and agreeableness on personality tests [6]. Their low agreeableness may account for the high prevalence of cyber victimization and cyberbullying perpetration in this population. Their low extraversion, in combination with their high neuroticism, may partially explain these users' low willingness to interact with others and authentically self-disclose on SNS. Conceptually, these personality traits make autistic users relatively safe from most social privacy and safety threats on SNS, except for cyberbullying. On the other hand, autistic users with comorbid mental illnesses 
— particularly, social anxiety and depression - may heavily rely on SNS to satisfy their social and emotional needs. As will be discussed in the next section, frequent SNS use can increase users' vulnerability to privacy and safety threats online.

\subsubsection{Frequency of Active Social Networking Site Use}

Several studies demonstrate the strong association between frequency of active SNS use and susceptibility to privacy violations $[4,60,61,76]$. Ang et al. [4] found that teenagers who frequently use SNS to communicate with others are more vulnerable to cyberbullying than less active users. Similarly, Leung [60] found that teenagers with symptoms of Internet addiction are more likely to self-disclose and be solicited for personal information by strangers on SNS than those who regulate their use.

Researchers attest autistic teenagers who use SNS to compensate for their offline social impairments are highly at risk of problematic Internet use. For instance, Benford and Standen [12] investigated the experiences, perceptions, and motivations of autistic people regarding Internet-based communication. They found that some of their participants used the Internet excessively for social communication because they were more comfortable communicating with others online than face-to-face. These findings suggest that some autistic users - particularly those who are lonely and socially anxious - may be at increased risk of encountering privacy violations on SNS.

\subsubsection{Previous Experience with Privacy Violations}

Research shows that previous experience with privacy violations has a positive influence on teenagers' thoughts and actions related to protecting their online privacy $[18,23,27]$. Christofides et al. [27] found that teenagers who had a negative experience on Facebook were motivated to learn about and use privacy settings to protect their personal information. Similarly, Bryce and Fraser [18] concluded that exposure to online risks plays an important role in teenagers' development of resilience to negative online experiences and effective coping strategies.

Autistic youth, especially those with intellectual disabilities, are often heavily guarded from online privacy and safety risks by their caregivers [21,48]. Meanwhile, 
researchers investigating Internet use by people with intellectual disabilities advocate for providing these users more opportunities to engage in positive risk-taking online $[22,127]$. For instance, Wasserman [127] argues that people with intellectual disabilities can learn valuable lessons from encountering and overcoming privacy risks on the Internet. Therefore, efforts must be made to shift current privacy-protective measures for this population from caregiver oversight to accessible, individualized security and privacy settings.

\subsubsection{Parental Mediation}

Parents play a crucial role in educating teenagers about privacy risks on SNS, and in protecting them from online threats. In general, parents use two strategies to support their child's online privacy protection and Internet literacy [4]:

1. Active mediation: Parents discuss the risks and benefits of Internet use with their children, and teach them how to protect themselves from online privacy and safety threats.

2. Restrictive mediation: Parents use restrictive measures (e.g., blocking websites) to limit their child's Internet use.

Parents' mediation strategies widely vary, based on their own privacy attitudes and behaviours (among several other factors) [117,119]. Nevertheless, research shows that active mediation is more effective at preventing teenagers from jeopardizing their online privacy and safety than restrictive mediation [63,129]. In fact, highly restrictive mediation may trigger teenagers to rebelliously engage in risky behaviours on SNS [94] and prevent them from learning how to cope with online risks independently [129].

Although some parents of autistic teenagers may use active mediation strategies, research suggests that they usually employ restrictive mediation strategies to defend their child from online privacy risks. Just and Berg [48] investigated caregivers' concerns and mediation strategies for keeping autistic children safe online. The caregivers were extremely anxious about their autistic children interacting with online predators; posting personal photos of their family, friends, and themselves on SNS; being exposed to explicit adult content; and engaging in inappropriate behaviours (e.g., creating accounts and using caregivers' credit card without permission) on the Internet. 
They were also concerned about their ability to effectively protect their child's safety online. The caregivers described autistic children as more "naive" than their typically developing peers, and that they therefore require greater protection than non-autistic users. In turn, the caregivers took strict measures to control their dependent's access to Wifi, devices, and accounts, such as confiscating the child's devices and disabling their accounts. They also closely monitored their child's online activities.

Research shows that parental mediation is a main protective factor against cyberbullying of autistic users $[50,130]$. Indeed, Wright [130] found that increased parental mediation and support were associated with fewer incidences of cyberbullying experienced by autistic teenagers during computer-mediated communications. However, research shows that these parents vary in their level of awareness of their child's online activities and experiences. Kowalkski and Fedina [50] investigated parents' understanding of their autistic child's experiences with cyberbullying. The researchers found that parents were fairly uninformed about their child's cyberbullying experiences, with 12 percent of the sample admitting that they did not know if their child had been bullied online. Moreover, only 9 percent of parents reported that they had ever discussed cyberbullying with their child.

\subsubsection{Online Privacy Educators}

Studies show mixed findings regarding teachers' influence on teenagers' privacy attitudes and behaviours on SNS. Shin and Lwin [95] found that teachers are more proactive in instructing teenagers how to use the Internet safely than parents. In contrast, Van Gool et al.'s [117] findings suggest that teachers do not influence teenagers' self-disclosure behaviours onine.

Little is known about the role of teachers in shaping autistic teenagers' privacy attitudes and behaviours. However, Löfgren-Mårtenson et al. [66] investigated teachers' views on Internet use by youth with intellectual disability for romantic and sexual purposes. The researchers found that teachers were more aware of their students' online social and sexual activities than the students' parents. In many cases, the teachers were responsible for helping students to distinguish between safe and risky personal information disclosure during computer-mediated communications. 


\subsubsection{Peer Influence}

Teenagers' friends and classmates have a substantial influence on their online social activities and, in turn, their privacy on SNS $[9,40,68,95,106,117]$. In many cases, this influence is indirect. For instance, although their peers may not demand that they remain available online, teenagers often feel pressured to be constantly active on SNS out of fear of becoming disconnected from their friends or missing out on rewarding experiences $[9,13,106]$. As previous discussed, excessive use of SNS often coincides

with higher chances of experiencing privacy violations $[4,76]$. Moreover, research shows that teenagers are more likely to engage in risky online behaviours when they seek advice or support from their peers about Internet-related privacy issues instead of consulting their parents or teachers $[94,95]$. However, peers may also have a positive influence on teenagers' privacy and safety on SNS, as evidenced by the success of peer support programs embedded within online privacy education [117].

It is possible that autistic teenagers' use of SNS and online privacy is influenced by their peers. However, considering that these users tend not to have many close friends [82], they are likely less susceptible to peer influence than the general population.

\subsubsection{Usability of Social Networking Sites}

Usability and privacy are intimately connected. In the following sections, we discuss some of the main usability issues of privacy settings on SNS and SNS in general, respectively, on autistic and non-autistic teenagers' privacy attitudes and behaviours.

\section{Usability of Privacy Settings}

Researchers have identified usability issues with the privacy settings on SNS for teenage users. Silva et al. [99] conducted a heuristic evaluation of the usability of privacy settings on Facebook for young people. They found severe violations of 4 of the 10 usability heuristics proposed by Nielsen [74], namely the match between the system and the real world (H2); consistency and standards (H4); recognition rather than recall (H7); and help and documentation (H10). Other studies shed light on teenagers' subjective perceptions of privacy settings on SNS that contribute to decision to use or avoid these tools. For instance, Snakenborg et al. [100] found that 
although teenagers are aware of the "blocking" tool on SNS, they are hesitant to use it to defend themselves against cyberbullying out of fear that this may cause the bullying to escalate. Similarly, Chen et al. [26] argue that teenagers rarely delete or block any of their contacts on SNS because doing so would be considered rude and may result in a loss of social capital.

Some research suggests autistic teenagers, especially those with intellectual disabilities, may have more difficulty using the privacy and security options on SNS than their typically developing peers [89]. Studies show that people with intellectual disabilities are often unaware of the privacy settings on SNS. For instance, one of the adults with intellectual disabilities in Holmes and O'Loughlin's [43] study was entirely unaware of the "block" feature on Facebook. Other studies highlight the challenges that these users encounter with using security tools on SNS, such as creating, memorizing and inputting text-based passwords [20]. In some cases, these usability issues undermined users' autonomy. For example, the young adults with intellectual disability in Kydland et al.'s [56] study had difficulty logging into their Flickr account because there are multiple ways of doing so on the site. In turn, participants required support to determine the most efficient way to gain access to their account.

Furthermore, cognitive accessibility issues with SNS may prevent autistic users from using the privacy settings on the platforms. Bahiss et al. [7] found that autistic users can be easily overwhelmed by the amount of information displayed on SNS. Considering that privacy settings are hidden on most SNS, autistic users may have difficulty finding these features on their account amid all of the other features and information on the platforms. Similarly, other researchers have remarked on the negative impact of interface updates on the usability of Facebook for adults with intellectual disabilities, who struggle to "re-learn" how to use the site following design changes $[96,98]$. These types of updates may make it difficult for autistic teenagers to keep track of the location of privacy tools on SNS.

\section{General Usability}

The overwhelming majority of studies examining teenage users' experiences with SNS, in general, mention little to no usability issues with the platforms. In contrast, several 
studies suggest that mainstream SNS are not usable for autistic users, particularly those with intellectual disabilities. In some cases, usability issues may have a positive impact on autistic teenagers' privacy, albeit at the expense of their social inclusion in digital communities. For instance, the text-based nature of computer-mediated communication often discourages autistic people from actively engaging in online social activities $[7,21,97,98]$.

On the other hand, accessibility issues with SNS may cause autistic users to inadvertently jeopardize their privacy. One of the main obstacles that people with intellectual disabilities face with using SNS is learning the terminology and social conventions of the platforms. Indeed, Shpigelman [96] found that the terms "timeline", "tag", "share", and "personal settings" were not intuitive for adults with intellectual disabilities. It is possible autistic users may have a different understanding of privacy-related terms (e.g., "privacy", "block", "report") on SNS than their typically developing peers. Similarly, Holmes and O'Loughlin [43] found that Facebook's use of the term "friend" was problematic for one of users with intellectual disabilities in their study because they interpreted the word in a very literal way. Motivated by a strong desire to increase their social capital, the participants accepted every "friend" request that they received. They ultimately added 600 Facebook "friends" whom they had never met before offline. Over time, the participant started to receive unwanted messages and negative comments about their appearance and activities from their Facebook "friends". However, they refused to block the users who were harassing them because they considered these people to be their actual friends and they did not want to lose them.

\subsection{Current Study}

Tables 2.1 and 2.2 offers a summary of the information provided in this Chapter. To date, little is known about autistic teenagers' privacy attitudes and behaviours on SNS. Despite a proliferation of studies investigating non-autistic teenagers' privacy concerns and protective strategies in online social contexts, researchers have yet to examine the experiences and perspectives of autistic teenagers with regards to privacy protection on SNS. Moreover, there is a paucity of research comparing the 
Table 2.1: Summary of SNS use, privacy attitudes, and privacy behaviours

\begin{tabular}{|c|c|c|}
\hline Topic & Autistic & Non-Autistic \\
\hline \multicolumn{3}{|c|}{ SNS Use } \\
\hline & $\begin{array}{l}\text { - Are "almost constantly" online } \\
\text { - Highly value SNS for facilitating commu- } \\
\text { nication, self-expression, and relationship } \\
\text { building } \\
\text { - SNS are beneficial for identity develop- } \\
\text { ment, social support, and well-being } \\
\text { - High risk of developing a controversial } \\
\text { digital footprint; organizational privacy } \\
\text { threats; unwanted attention; exposure to } \\
\text { inappropriate content; and cyberbullying }\end{array}$ & $\begin{array}{l}\text { - Use SNS less frequently than the general } \\
\text { population } \\
\text { - Most rarely use SNS for social communi- } \\
\text { cation; however, some rely on these plat- } \\
\text { forms to make friends } \\
\text { - SNS are especially beneficial for decreas- } \\
\text { ing loneliness and social isolation } \\
\text { - Potentially more vulnerable to online pri- } \\
\text { vacy threats than the general population } \\
\text { - Very high risk of cyberbullying }\end{array}$ \\
\hline \multicolumn{3}{|c|}{ Privacy Attitudes } \\
\hline & $\begin{array}{l}\text { - Mostly aware of social privacy threats } \\
\text { - Wide variability in willingness to trade } \\
\text { privacy for social benefits }\end{array}$ & $\begin{array}{l}\text { - Unknown } \\
\text { - Potentially high privacy concerns }\end{array}$ \\
\hline \multicolumn{3}{|c|}{ Privacy Behaviours } \\
\hline & $\begin{array}{l}\text { - Engage in risk-taking to fulfill social and } \\
\text { emotional needs } \\
\text { - Privacy protection strategies include: se- } \\
\text { lecting audiences; selecting platforms; se- } \\
\text { lecting accounts; limiting screenshots; ne- } \\
\text { gotiating consent; deleting photos; }\end{array}$ & $\begin{array}{l}\text { - Unknown } \\
\text { - Probably have more difficulty protecting } \\
\text { their privacy on SNS than the general } \\
\text { population }\end{array}$ \\
\hline
\end{tabular}

factors affecting autistic teenagers' privacy attitudes and behaviours on SNS with those influencing their typically developing peers. We contribute to the literature by providing preliminary insight into the challenges that autistic teenagers encounter with protecting their privacy on SNS. The findings from this study will inform the development of balanced interventions supporting these users' privacy protection and participation on these platforms. 
Table 2.2: Summary of factors affecting privacy attitudes and behaviours

\begin{tabular}{|c|c|c|}
\hline Topic & Autistic & Non-Autistic \\
\hline Age & $\begin{array}{l}\text { - Greater risk-taking among older teenagers than younger ones, due to } \\
\text { higher self-efficacy and autonomy }\end{array}$ & $\begin{array}{l}\text { - Unknown } \\
\text { - Increase in psychiatric disorders in older adolescence can lead to } \\
\text { problematic Internet use and elevated exposure to privacy threats } \\
\text { - Improvements in social skills in older adolescence may decrease } \\
\text { cyberbullying }\end{array}$ \\
\hline Gender & $\begin{array}{l}\text { - Females have greater privacy concerns and use privacy settings } \\
\text { more than males, but they also post more sensitive content } \\
\text { - Higher rates of online aggression among males compared to females }\end{array}$ & - Unknown \\
\hline Disposition & $\begin{array}{l}\text { - SNS use and online privacy vary depending on personality (i.e., Big } \\
\text { Five traits) and mental health status (e.g., social anxiety, depres- } \\
\text { sion) }\end{array}$ & $\begin{array}{l}\text { - Combination of personality traits (i.e., low agreeableness, low } \\
\text { extroversion, high neuroticism) makes them resilient to privacy } \\
\text { threats, except cyberbullying } \\
\text { - High social anxiety and depression may lead to problematic In- } \\
\text { ternet use and, in turn, high vulnerability to privacy violations }\end{array}$ \\
\hline $\begin{array}{l}\text { Frequency of } \\
\text { active SNS use }\end{array}$ & $\begin{array}{l}\text { - Higher active SNS use coincides with greater risk of privacy viola- } \\
\text { tions }\end{array}$ & $\begin{array}{l}\text { - Some autistic people use SNS excessively, thereby increasing their } \\
\text { vulnerability to privacy violations }\end{array}$ \\
\hline $\begin{array}{l}\text { Previous expe- } \\
\text { rience with pri- } \\
\text { vacy violations }\end{array}$ & $\begin{array}{l}\text { - Exposure to online risks can lead to resilience and promote the } \\
\text { development of effective coping strategies }\end{array}$ & - Heavily guarded from privacy threats \\
\hline $\begin{array}{l}\text { Parental } \\
\text { mediation }\end{array}$ & $\begin{array}{l}\text { - Two types of mediation: active (privacy advice) or restrictive (lim- } \\
\text { iting SNS use) } \\
\text { - Active mediation is best }\end{array}$ & $\begin{array}{l}\text { - Parents typically use restrictive mediation } \\
\text { - Parental mediation is crucial for preventing cyberbullying }\end{array}$ \\
\hline $\begin{array}{l}\text { Online privacy } \\
\text { educators }\end{array}$ & $\begin{array}{l}\text { - Teachers may or may not substantially impact teen's privacy atti- } \\
\text { tudes and behaviours }\end{array}$ & $\begin{array}{l}\text { - Unknown } \\
\text { - Teachers may be more aware of autistic teens' online activities } \\
\text { than parents }\end{array}$ \\
\hline Peer influence & $\begin{array}{l}\text { - Substantial influence on online activities } \\
\text { - May have positive or negative impact on online privacy }\end{array}$ & $\begin{array}{l}\text { - Unknown } \\
\text { - Probably low impact on online privacy }\end{array}$ \\
\hline $\begin{array}{l}\text { Usability } \\
\text { of SNS }\end{array}$ & $\begin{array}{l}\text { - Design of privacy settings violates usability heuristics, and } \\
\text { teenagers have negative attitudes towards these features } \\
\text { - No substantial usability issues with SNS in general }\end{array}$ & $\begin{array}{l}\text { - Notable cognitive accessibility issues with privacy settings and } \\
\text { SNS in general }\end{array}$ \\
\hline
\end{tabular}




\section{Chapter 3}

\section{Methodology}

We conducted semi-structured interviews with autistic and non-autistic teenagers to answer the following research questions:

RQ1: What are autistic teenagers' thoughts and feelings about their privacy on SNS, and how do they protect their online privacy?;

RQ2: What are the main factors affecting autistic teenagers' privacy attitudes and behaviors on SNS?; and

RQ3: How do these privacy-related attitudes, behaviours, and factors compare with those reported by non-autistic teenagers?

To provide context for our study's findings, we provide an overview of participants' demographic characteristics. We also describe our materials and our recruitment, data collection and analysis processes.

\subsection{Participants}

\subsubsection{Recruitment}

Following ethics clearance from Carleton University's Research Ethics Board, we recruited autistic and non-autistic people across Canada who were between 13 to 17 years in age; used Twitter, Instagram, or Facebook (three of the most popular SNS among teenagers in North America [3]); and were able to speak English and participate in an interview with or without support. Due to the hard-to-reach nature of our target demographics, we recruited participants through several avenues: (1) we emailed 46 local autism-related organizations, asking them to share our study's recruitment information with their members; (2) we posted our recruitment information in Facebook groups that are relevant to our target demographics (e.g., homeschooling in Ottawa, autism support groups across Canada); (3) we placed posters in all 
Table 3.1: Summary of participant demographics

\begin{tabular}{lrr}
\hline & $\begin{array}{r}\text { Autistic } \\
(\mathrm{n}=12)\end{array}$ & $\begin{array}{r}\text { Non-Autistic } \\
(\mathrm{n}=16)\end{array}$ \\
\hline Gender (\%) & & \\
Female & 33 & 50 \\
Male & 58 & 50 \\
Non-binary & 8 & 0 \\
\hline Age in years (M(SD)) & $(1.6)$ & $14.7(1.3)$ \\
\hline Preferred SNS (\%) & 67 & \\
Instagram & 25 & 88 \\
Facebook & 8 & 0 \\
Twitter & 50 & 12 \\
\hline Frequency of SNS use (\%) & 25 & 94 \\
Every day & 8 & 6 \\
A few times per week & 8 & 0 \\
Once per week & & 0 \\
Less than once per week & 50 & \\
\hline SNS Profile Privacy Setting (\%) & 42 & 31 \\
Public & 8 & 56 \\
Private & & 12 \\
Private \& Public & & \\
\hline Device used to go online $(\%)$ & 75 & 100 \\
Smartphone & 25 & 6 \\
Tablet & 75 & 25 \\
Laptop & 42 & \\
Desktop computer & 8 & \\
Gaming system & & \\
\hline Access to Internet at home (\%) & & \\
Yes & & \\
No & & \\
\hline & & \\
\hline
\end{tabular}

public libraries in Ottawa and around Carleton University's campus; (4) we sent our recruitment information to staff members in the School of Computer Science at Carleton University who were subscribed to an internal emailing list; and (5) we encouraged participants to share our study's recruitment information with their family and friends. Our recruitment materials are included in Appendix A.

\subsubsection{Demographics}

Tables 3.2 and 3.1 present participants' demographic characteristics. 
Table 3.2: Detailed participant demographics and preferred SNS

\begin{tabular}{|c|c|c|c|c|}
\hline Group & Participant ID & Age & Gender & Preferred SNS \\
\hline \multirow[t]{16}{*}{ Non-Autistic } & NA1 & 14 & Female & Instagram \\
\hline & NA2 & 14 & Male & Instagram \\
\hline & NA3 & 16 & Female & Instagram \\
\hline & NA4 & 15 & Female & Twitter \\
\hline & NA5 & 15 & Male & Instagram \\
\hline & NA6 & 16 & Female & Twitter \\
\hline & NA7 & 17 & Female & Instagram \\
\hline & NA8 & 16 & Male & Instagram \\
\hline & NA9 & 13 & Male & Instagram \\
\hline & NA10 & 14 & Female & Instagram \\
\hline & NA11 & 14 & Male & Instagram \\
\hline & NA12 & 14 & Male & Instagram \\
\hline & NA13 & 13 & Female & Instagram \\
\hline & NA14 & 17 & Male & Instagram \\
\hline & NA15 & 13 & Male & Instagram \\
\hline & NA16 & 15 & Female & Instagram \\
\hline \multirow[t]{12}{*}{ Autistic } & A1 & 15 & Female & Instagram \\
\hline & $\mathrm{A} 2$ & 16 & Male & Facebook \\
\hline & A3 & 15 & Male & Instagram \\
\hline & $\mathrm{A} 4$ & 16 & Non-binary & Instagram \\
\hline & A5 & 15 & Female & Instagram \\
\hline & A6 & 17 & Male & Facebook \\
\hline & $\mathrm{A} 7$ & 15 & Male & Facebook \\
\hline & A 8 & 13 & Female & Instagram \\
\hline & A 9 & 14 & Male & Instagram \\
\hline & A10 & 15 & Male & Instagram \\
\hline & A11 & 15 & Female & Instagram \\
\hline & A12 & 13 & Male & Twitter \\
\hline
\end{tabular}




\section{Autistic}

Twelve autistic teenagers participated in our research. Most participants were male and above 14 years of age. Of the three SNS involved in this study, most autistic participants preferred Instagram. Half used their preferred site every day, whereas the others used it less frequently. Half had a public account. The other half had their account set to private or had separate private and public accounts. Most autistic participants reported using a smartphone and/or laptop to go online. All autistic participants had Internet access at home.

\section{Non-Autistic}

Sixteen non-autistic teenagers completed our study. There were even gender (i.e., males and females) and age (i.e., younger and older) distributions in our non-autistic sample. The vast majority of non-autistic participants preferred Instagram over the two other SNS involved in our research. Nearly all used their preferred site every day, and most had a private account. All non-autistic participants used a smartphone to go online and had Internet access at home.

\subsection{Materials}

\subsubsection{Interview Guide}

The interview guide for the study is included in Appendix C. The first part of the interview consisted of scenarios with follow-up questions about participants' reactions and experiences with different privacy-related situations on SNS (see section 3.2.2 for details). The second part of the interview included questions about participants' use of SNS and their thoughts about online privacy. The interview concluded with basic demographic questions (e.g., age, gender, devices). The interview guide was reviewed and approved by an expert in conducting research with people with intellectual and developmental disabilities prior to data collection. 


\subsubsection{Scenario Video Clips}

To examine participants' privacy attitudes and behaviours on SNS, we developed five scenarios reflecting authentic situations related to privacy protection in online social contexts (see Appendix $\mathrm{C}$ for transcripts of the scenarios). Autistic people greatly benefit from visual stimuli to enhance their attention, comprehension, and learning [83]. To make our study as accessible as possible, we presented the scenarios as 13- to 20-second videos on the interviewer's laptop. The videos feature the voice and profile of a fictional teenage girl who is looking for advice about how to protect her privacy on SNS. Considering that the scenarios were only intended to serve as initial prompts for discussion about participants' own attitudes and behaviours, we expected the character's gender to have a negligible effect on our data. We created comparable videos for three SNS involved in our study. The following subsections describe the content and theme of each scenario. Figures 3.1 and 3.2 offer screenshots of the Instagram version of each scenario.

\section{Scenario 1: General Self-Disclosure}

In this scenario, the character announces that she got a new puppy and shows a picture of the dog. She asks with whom she should share the picture. This scenario served as a prompt for further questions assessing participants' attitudes towards disclosing personal content (particularly photos) on SNS. Specifically, we prompted for who participants wanted to see their content and any privacy concerns they may have with posting personal content on their profile.

\section{Scenario 2: Self-Disclosing to Strangers}

In this video clip, the character explains that someone she does not know started following her account. She displays the person's profile on screen. She asks for advice about what she should do in this situation. The aim of this scenario was to understand participants' views on allowing strangers to see their content on SNS. We also wanted to assess participants' interest in interacting with strangers and making friends online. 


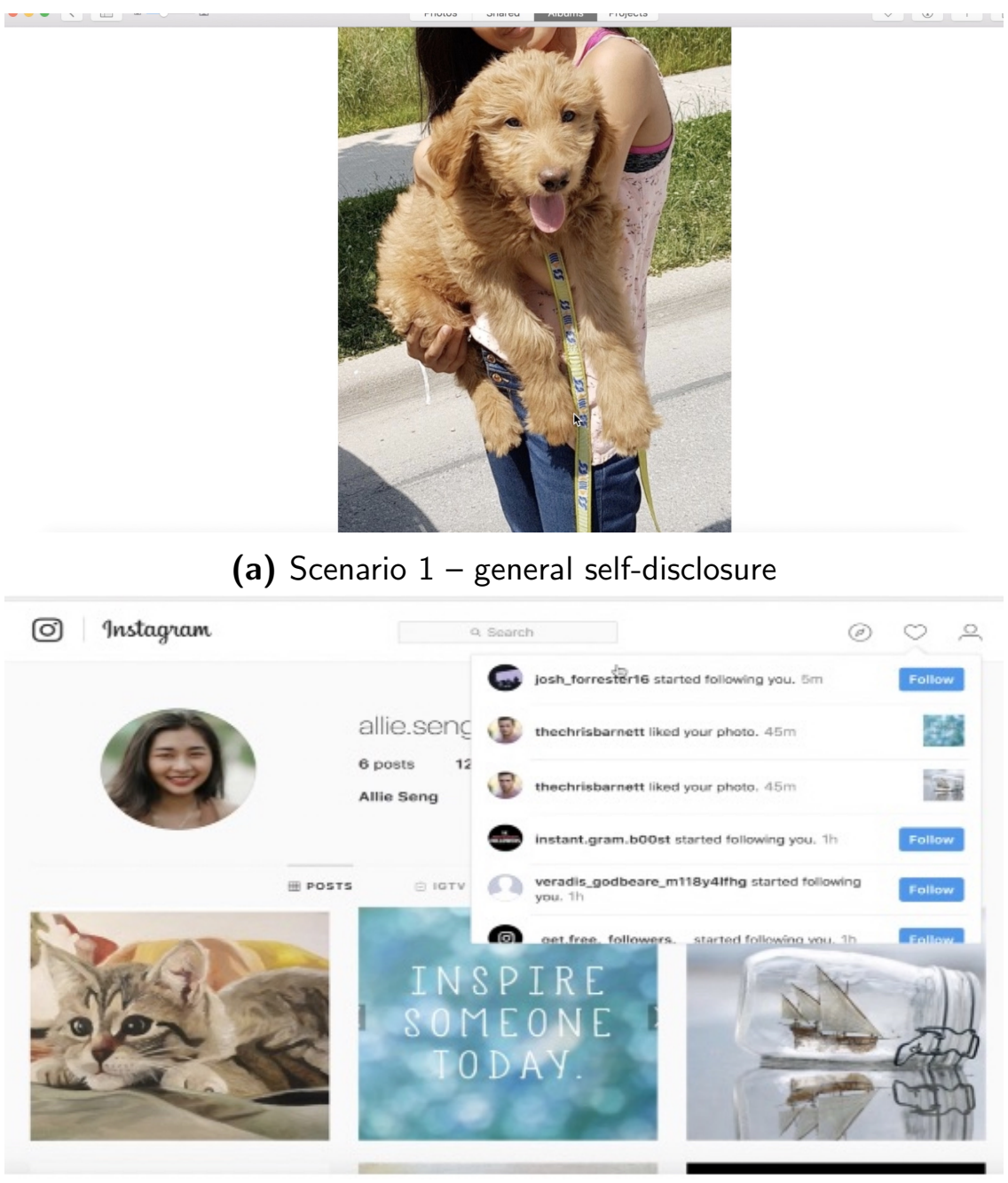

(b) Scenario 2 - self-disclosing to strangers
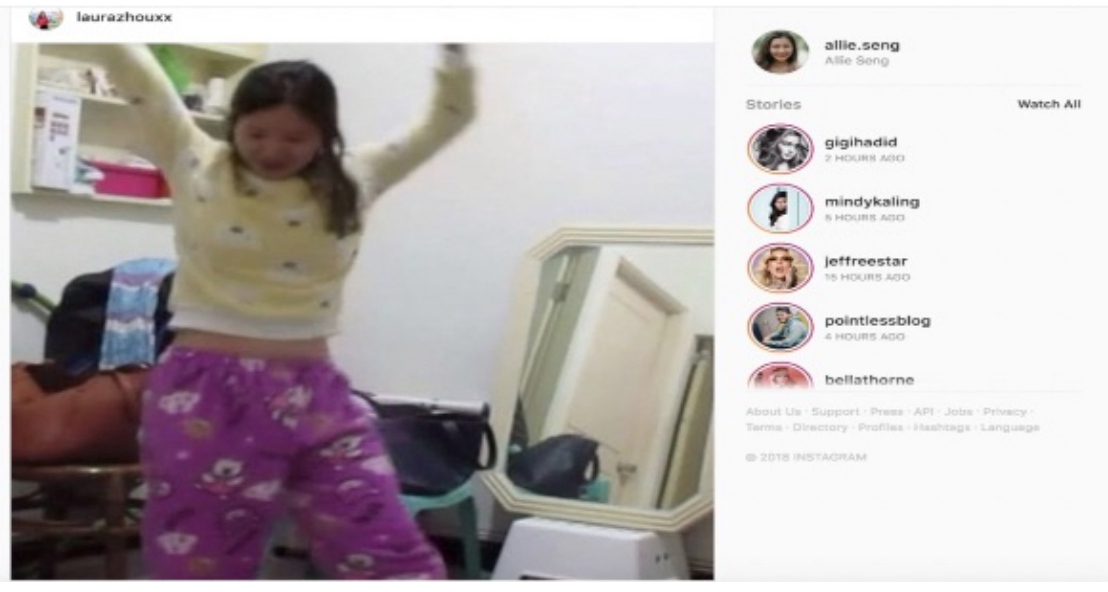

(c) Scenario 3 - non-consensual disclosure by others

Figure 3.1: Screenshots representing Scenarios 1-3 from Instagram. 


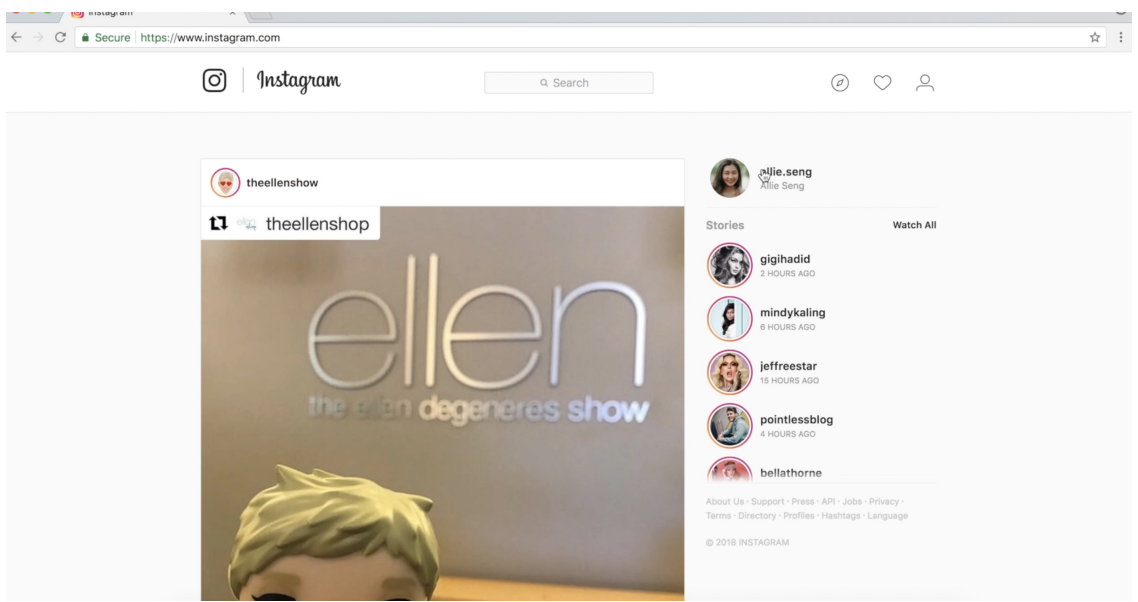

(a) Scenario 4 - using privacy settings

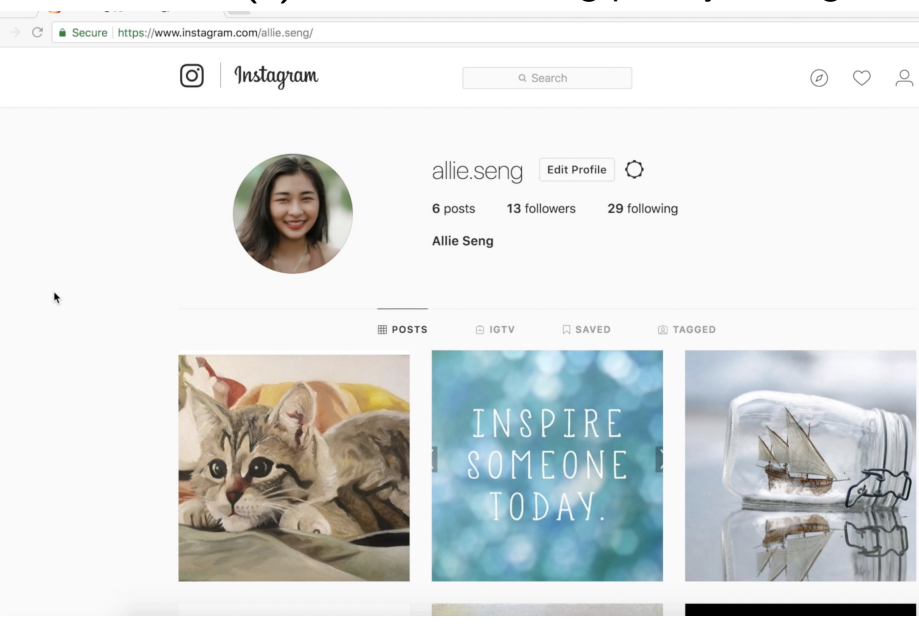

(b) Scenario 5 - other privacy protection strategies

Figure 3.2: Screenshots representing Scenarios 4-5 from Instagram. 


\section{Scenario 3: Non-Consensual Disclosure by Others}

In this scenario, the character says that her friend posted an embarrassing photo of her online. The photo is of the character dancing in her pajamas. She wants to know what she should do in this situation. The objective of this scenario was to examine participants' strategies for dealing with non-consensual disclosure of personal information by others on SNS. This scenario also prompted discussion about participants' experiences with cyberbullying.

\section{Scenario 4: Using Privacy Settings}

In this video, the character explains that she does not know how to set her account to private; she asks participants if they know how to do it. The main objective of this scenario was to assess the usability of the private account settings on SNS. To do this, we provided participants who completed the study in-person access to the character's account and asked them to make it private. We took observational notes of any difficulties that participants encountered.

\section{Scenario 5: Other Privacy Protection Strategies}

The aim of this scenario was to provide participants the opportunity to discuss other strategies that they use to protect their privacy on SNS. In the video, the character asks if there is anything else that participants do to keep their personal information safe.

\subsection{Procedure}

Interested participants contacted the lead researcher to select a time and location to complete the semi-structured interview, and to indicate their preferred SNS (i.e., Instagram, Facebook or Twitter). The interviews took place in an Ottawa public library $(n=16)$, in a private office space on Carleton University's campus $(n=7)$, or through Skype $(n=5)$. At the beginning of each session, participants' parents consented to their son or daughter's involvement in the study by signing an informed consent form (see Appendix B), and participants provided verbal assent to complete 
the interview and to be audio-recorded (see Appendix C). None of the participants required assistance to participate. However, three of the participants' parents were seated within hearing distance of the interview, which may have influenced these participants' responses. During the sessions, participants watched the scenario video clips for their preferred SNS (22 saw Instagram; 3 saw Twitter; 3 saw Facebook) and answered the questions in the interview guide in conversation with the researcher. Finally, they were debriefed and compensated with 30 dollars for their time. The sessions varied from 20 to 60 minutes in length.

\subsection{Qualitative Data Analysis}

Following data collection, all of the audio recordings were transcribed verbatim and imported into Atlas.ti ${ }^{1}$ software for analysis. The lead researcher conducted a thematic analysis [17] on autistic and non-autistic participants' interview transcripts separately. Thematic analysis is a "theoretically flexible" [17] approach that is appropriate for exploratory research. First, the lead researcher reviewed the transcripts several times to become familiar with the data and to identify preliminary trends in participants' responses. Then, descriptive labels or "codes" were applied to sections of the data. Afterwards, similar codes were grouped into broader themes (e.g., social factors) and sub-themes (e.g., support, influence). Finally, the lead researcher conducted side-by-side descriptive comparisons of the codes/themes in each group's transcripts, identifying key similarities and differences in the independent samples' trends. A second coder analyzed 20 percent of the transcripts so that the lead researcher could assess the reliability of the coding structure and make adjustments as required. Overall, we found good reliability between the two raters in that they both applied many of the same codes to corresponding sections of the transcripts. However, the lead researcher frequently applied more codes to the qualitative data than the secondary coder. The lead researcher reconciled differences in the coding as necessary. Ultimately, approximately 12 hours of audio-recordings were manually transcribed, and 1334 individual quotations were coded.

\footnotetext{
${ }^{1}$ https://atlasti.com/
} 


\section{Chapter 4}

\section{Results}

In this chapter, we present the findings from our semi-structured interviews with autistic and non-autistic teenagers. We first describe and compare the privacy attitudes and privacy behaviours of participants in each group. We then discuss factors affecting participants' privacy-related thoughts and actions on SNS.

\subsection{Privacy Attitudes}

We found many similarities and differences between autistic and non-autistic participants' thoughts and emotions regarding their privacy on SNS. We provide an overview of the privacy attitudes of participants in each group, specifically their knowledge and awareness of privacy threats; privacy concerns; and considerations towards balancing privacy and self-disclosure online.

\subsubsection{Knowledge and Awareness of Privacy Threats}

\section{Non-Autistic}

As expected, most non-autistic participants appeared to be conscious of social privacy threats (e.g., cyberbullying, cyberstalking, catfishing) on SNS; however, they were relatively oblivious to other types of privacy and security risks inherent to online social networking. In general, non-autistic participants did not seem to be aware of the fact that SNS companies can easily access and sell their data to third-party advertisers without their knowledge or voluntary consent. When asked who can see their content on SNS, none of the non-autistic participants acknowledged the companies hosting the sites or advertisers; they exclusively mentioned other users (i.e., friends, family, strangers). Participants' incomplete mental model of who can view their personal information on SNS not only related to their profile posts, but also their account 
credentials (i.e., passwords, emails, phone numbers). For instance, one participant believed that no one can see the phone number and email address associated with their account due to privacy settings:

NA12: Having a private account makes sure that no one can access your phone number, if you have one on there. No one can access your email, if you have one on there. No one can see who follows you. Just making a private account works.

Interviewer: Okay. So, you have a phone number or email address associated with your account.

NA12: I do. But no one can access it without asking me for it.

In reality, social media companies have the power to not only access all users' account credentials and posted content, but also to use it at their discretion, within the limits of their Terms of Service and Privacy Policy.

Similarly, non-autistic participants did not know about data mining programs, which can covertly collect data on their account. For instance, when discussing reputation threats on SNS, one participant noted: "Even if you do take the post down, there's the chance that somebody somewhere has saved that. So, now it's in their hands." (NA11). The participant assumed that this type of privacy risk is always caused by a vengeful user or online predator saving a specific piece of personal information to blackmail or humiliate their victim. They did not seem to be aware of technology-mediated data collection, such as data profiling or web crawling.

Moreover, non-autistic participants believed that they were more anonymous on SNS than in reality. For instance, one participant explained that they did not have any personal photos on their Instagram profile and therefore felt like they did not have a traceable identity on the site: I really don't post anything on Instagram [...] I'm just there to talk to my friends, see their posts - I'm just a ghost (NA15). Due to their perceived anonymity, the participant was comfortable allowing strangers to follow their public account, explaining: If I don't know them, it wouldn't be a problem because, well, they probably don't know me either (NA15). They did not seem to 
realize that their identity may be uncovered through their affiliations or other covert identifiers (e.g., IP address).

\section{Autistic}

In previous research [48], autistic users have been described as more naive to online privacy threats than the general population. Interestingly, the autistic participants in our sample did not seem to be less aware of the risks inherent to personal information disclosure on SNS than non-autistic participants. In fact, one autistic participant (who had a special interest in computer programming) demonstrated greater awareness of organizational privacy threats than their typically developing counterparts:

A12: I don't like that companies track me. I really don't [...] I feel often that they know more about me than I know about myself often. And, like, should a big company really know this much about you? Like, they're basically using you for money, and then they don't really care who you are. They just care about your personal information.

Nevertheless, the vast majority of autistic participants had equally limited knowledge of how their personal information may be inappropriately accessed and used as non-autistic participants. Similar to their typically developing counterparts, autistic participants were almost exclusively conscious of easy-to-understand and wellpublicized social privacy threats related to online social networking, such as cyberstalking, cyberbullying, catfishing, and hacking.

\subsubsection{Privacy Concerns}

\section{Non-Autistic}

Most non-autistic participants expressed low or moderate concern about privacy and safety threats online. A pervasive pattern in the data is that non-autistic participants tended to downplay perceived risks associated with online social networking. Although all non-autistic participants were at least somewhat wary of potential predators on SNS, the majority believed that they probably would never be targeted by 
these individuals. For instance, one non-autistic participant explained that they do not disclose their full name on Instagram to protect themself from being stalked:

Interviewer: Why don't you want people to know your name?

NA13: Just because then they can Google me and then they can figure out where I live and then they'd stalk me.

However, the participant believed that it was unlikely that anyone would stalk them, stating: That's probably not going to happen (NA13). Another non-autistic participant described that they were careful not to post offensive content on their profile to ensure that no one could use their posts against them:

NA11: In theory, if somebody were to take a look at what you post and later on give examples, you could be out of a job, you could be out of a college, you could be out of a university. Your life could take a drastic turn backwards, just because you posted a funny image that offended somebody.

Nevertheless, they went on to describe this concern as "more paranoid than realistic"(NA11). Similarly, when asked why they do not disclose their contact information on SNS, one participant replied: You could get scammed or have your identity stolen. Like, it's extreme cases, of course, but you still have to be cognizant of that (NA3).

Moreover, some non-autistic participants (particularly the older teenagers) were comfortable allowing strangers to follow their account, as long as the follower did not exhibit any behaviours that they deemed inappropriate or creepy: I have people who I definitely don't know who follow me. And as long as they don't like get in my business or try to interact with me in a way that I don't like, then I don't really care (NA7). Similarly, some non-autistic participants had online-only friends who they talked to about their personal life without concern about these unknown users' intentions:

Interviewer: Do you have any followers on Instagram who you don't know in real life?

NA8: Yes, a couple. Most of them I don't know in real life, but I've spoken to them often through like voice calls and stuff. Or they're like friends of like my cousins, who live elsewhere. 
Interviewer: Do you consider those people to be your friends?

NA8: Yeah, they're great.

These findings are in line with the previous research demonstrating that teenagers without autism are mindful of predators on SNS but tend to feel safe online [1].

Furthermore, non-autistic participants were unconcerned about their online privacy and safety being threatened by their peers. None of the non-autistic participants were worried about their friends posting photos of them without their consent. They trusted their friends to protect their privacy and to abide by their implicit or explicit disclosure preferences on SNS. For instance, one non-autistic participant said: $M y$ friends know that I don't like to show my face on Instagram, so they don't usually put it on (NA10). Moreover, none of the non-autistic participants appeared to be concerned about being bullied on SNS. This may be partially due to their idealistic perceptions that their profile cannot or will not be accessed by people they do not trust. As one non-autistic participant explained: I'm friends with the people I follow. And I guess the people who dislike me, they don't really follow me. (NA11).

\section{Autistic}

In general, the majority of autistic participants were highly concerned about their privacy and safety on SNS. Unlike most participants in the non-autistic group, several autistic participants discussed extreme privacy and safety risks on SNS caused by online predators with genuine fear of being targeted. For instance, one autistic participant did not post their location or photos of themself online because they were afraid of being recognized and kidnapped: So, strangers see the similar faces. They see them on the streets, "oh look! You're from Facebook" and you could get kidnapped or something. That's what I'm afraid of (A2). Similarly, another autistic participant was worried about being robbed if they disclosed their location on SNS: There are some bad people on the Internet. They can go over to your house and rob you by finding out your location (A11). Additionally, a few autistic participants were afraid of their account being hacked:

A2: If you share [photos] with everybody, something wrong can happen 
with it.

Interviewer: Like what?

A2: Like, you know, there's a hacker online.

Moreover, many autistic participants were wary of catfishing. When asked if the character in the scenario video clips should allow a stranger to follow her account (see Appendix C: Scenario 2), several autistic participants were highly skeptical of the unknown follower's identity and intentions:

A8: Don't let him follow her. He doesn't know her at all, and it could be someone that's just set up to act like someone else.

A10: If it's just random people she doesn't know, they can say they're like 14 and go to the same school or something, but they could be like 50 and just want to like murder her or something maybe. They could just be not who they say they are. You never know!

Partially due to these concerns, most autistic participants did not feel comfortable self-disclosing personal information to people they do not know on SNS.

Furthermore, autistic participants were slightly more concerned about their online privacy and safety being threatened by people they know in real life than their typically developing counterparts. Unlike the participants in the non-autistic group, some autistic participants were concerned about being bullied on SNS, particularly by their offline peers. For instance, when asked if the character in the scenario videos should post a picture of her dog online (see Appendix C: Scenario 1), one autistic participant - who reported being bullied in real life - was worried that some users may leave negative comments on the post:

Interviewer: Do you think that she should share this picture on Instagram?

A11: Maybe. Different things can happen depending on like who people are.

Interviewer: Like what kinds of things? 
A11: People could say rude things about the picture, even though it's like a nice happy one.

Nevertheless, autistic participants were not concerned about their privacy being threatened by their close friends. Much like typically developing teenagers, autistic participants trusted their friends to respect their privacy preferences.

\subsubsection{Balancing Privacy and Self-Disclosure}

\section{Non-Autistic}

Consistent with previous studies $[1,101]$, the non-autistic participants in our sample highly valued their privacy online. Although most were not necessarily concerned about privacy and safety threats on SNS, nearly all non-autistic participants expressed a desire to maintain some boundaries with different real or imagined audiences (refer to section 4.3 .3 for more details). At the same time, all non-autistic participants (except for one) placed a moderate or high degree of importance on developing an authentic profile and interacting with others on SNS. In other words, basically all non-autistic participants belonged in group 2, 3 or 4 of Soffer and Cohen's [101] classification of teenagers' privacy perceptions, with most of them in group 3.

Some non-autistic participants actively used SNS to post photos related to their hobbies, interests and experiences; however, they were uncomfortable publicly posting personally identifying content (e.g., photos of themselves, full name) online. For instance, a male participant explained that he did not post photos of himself on SNS simply because he "always found it a little weird" (NA14). He also did not disclose any other personally identifying information (e.g., contact information, name) and had a private account. Nevertheless, he enjoyed sharing funny memes and showcasing his hobbies (e.g., guitar playing) on his profile. This participant - along with other group 2 members - prioritized privacy over authentic self-disclosure on SNS.

Other (group 3) non-autistic participants were comfortable posting personal information on SNS, but only because their profile was private. For example, a female participant was not concerned about sharing photos of herself due to privacy settings:

Interviewer: Do you post pictures of yourself on Instagram? 
NA13: I do, yes.

Interviewer: And who do you want to see those pictures?

NA13: Well, the people that are following me, because I have a private account. So, people that are just kind of like "oh, who's this?", they can't see what I post.

Interviewer: Is there anyone who you would not like to see your pictures?

NA13: Well, random people. But out of the people that are following me, no.

Participants like this one placed equal importance on privacy and developing an authentic profile.

In contrast, a few non-autistic participants were very liberal in their self-disclosure on SNS. These participants openly disclosed personally identifying information on their public profile. For instance, one female participant was comfortable with anyone seeing her profile; she did not feel the need to take any measures to restrict access to her content unless triggered to do so:

Interviewer: Who do you want to see your pictures?

NA1: I really don't mind. Personally, my account is public, so I don't really mind who sees it. But if I see something sketchy, I would block the account or erase the comment or whatever.

This participant and others belonging in group 4 prioritized authentic self-disclosure over privacy protection.

\section{Autistic}

Overall, autistic participants were much more risk averse than their non-autistic counterparts. In stark contrast to the non-autistic sample, a large proportion of autistic participants belonged in group 1 or 2 of Soffer and Cohen's [101] classification of teenager's privacy perceptions, with most of them in group 2. A couple of autistic participants met the criteria for group 3, and only one belonged in group 4. In general, the vast majority of autistic participants did not seem to place a high value 
on developing an authentic online profile. In the trade-off between privacy and selfdisclosure, privacy was usually prioritized over active engagement on SNS. Many autistic participants very infrequently posted personal content on SNS, mainly using the platforms to view and provide feedback other users' posts (group 1). For instance, when asked what they do on Instagram, an autistic participant replied: I browse, I give a like, I comment. Done (A9). Most autistic participants occasionally posted on SNS, but exclusively about their interests, hobbies or experiences and with a private profile (group 2). Very few were comfortable sharing personal information, privately or publicly (group 3 and 4), preferring to feel relatively anonymous on SNS.

\subsection{Privacy Behaviours}

Autistic and non-autistic participants used a variety of strategies to protect their privacy on SNS. The most common privacy behaviours reported by both groups were: (1) deciding who to trust, (2) managing access, (3) negotiating consent, and (4) filtering out personal information.

\subsubsection{Deciding Who to Trust}

One of the greatest challenges that both autistic and non-autistic participants faced with protecting their privacy on SNS was deciding who outside of their offline friend group they can trust online. Autistic and non-autistic participants described strategies that they use to detect possible online predators. Some participants said that they checked their followers' profiles for suspicious or inappropriate content. In these cases, both autistic and non-autistic participants relied on complex heuristics, such as gut instincts and first impressions, to determine if the stranger can be trusted. For instance, one non-autistic participant allowed two unknown accounts to follow them because they approved of the content that the accounts posted:

NA6: One of [the unknown accounts] was like a platform for feminism, so I was okay with that. And there's another one that just shows pictures around the world, so I didn't think of anything much of it. 
Similarly, one autistic participant described that they examined their followers' account for sexual content:

A4: I looked at [my followers'] Instagrams to see if they're okay or if I should block them or something. And I'm like, "okay, they're fine. They just post memes like a normal teenager". If I see something that's too sexual, like bathing suit pics, but like too many of them - I don't mind one like showing off you got a new bathing suit. Yeah, it's cute and all that. But having like 40 of them, I'm like "no, no. That's a lot".

Some autistic and non-autistic participants were comfortable with allowing anyone to follow their account, as long as they did not give them a "bad vibe" or do anything overtly creepy, such as liking or commenting on too much of their content or sending them unsolicited direct messages:

NA7: If the communication is kind of out of nowhere and it's very forward and abrupt, I don't want that. Obviously, everyone has ghost followers that don't really interact with your page. So, if I had someone who was following me like that, and then all of a sudden they decided to try to talk to me, I would be kind of weirded out. Like, you never "liked" any of my pictures or anything like that. So, I would be weirded out.

A4: I just have gut feeling sometimes - when you like see someone and you're just like "that person gives me a bad vibe and I don't like them".

Meanwhile, other autistic and non-autistic participants decided that the safest course of action is not to trust anyone who they do not know online:

NA3: I think Instagram is a platform where you should share your everyday things - somewhere you can document it. Sharing that with someone who you don't know at all, it's not really important and it's not really safe. A12: I just don't really like talking with people I don't really know personally. And it's just easier if I have friends who I actually know, instead of online friends where they can be anybody. 
Overall, we only found one notable difference in how autistic and non-autistic participants decided who to trust on SNS. Many non-autistic participants said that they would seek out more information by trying to get to know their unknown followers and asking them about their intentions for following their account:

NA1: A lot of people that would follow me, I kind of heard of everyone. Like, I kind of have an idea who it is. But if I'm really unsure, then I would ask "who are you?".

NA12: Get to know this person. Like eventually DM them like "who are you?", that kind of stuff. Or "how did you find me?" - questions like that. I've done that before.

None of the autistic participants used this strategy.

\subsubsection{Managing Access}

\section{Privacy Settings}

Both groups used privacy settings (e.g., private account, reporting, blocking) as a main line of defense against privacy violations on SNS. More than half of autistic and non-autistic participants reported that they restrict access to their profile to prevent people they do not know from accessing their personal content:

NA9: I post but my account is on private, so only the people that I know see it.

A10: I decided I just want people that I know to follow me, so I made it private.

A few participants in each group also previously blocked or removed followers and reported inappropriate content on SNS. For instance, some participants said that they blocked accounts that sent them unsolicited messages:

NA10: I made sure to block them as soon as I got it.

A3: I just block them. 


\section{Multiple Accounts}

A few participants in each group reported having both a public and private account on their preferred SNS. These participants posted different content on each of their accounts in order to carefully curate their private and public persons. Generally, the participants used their public account to showcase their talents or to promote their business endeavours, whereas their private account was for personal photos. For instance, one non-autistic participant had a public account to advertise their photography services, and a private account for their own entertainment:

NA16: My non-private account [is] just for like if I want to post really nice pictures. And my private account is just for stupid posts that I find funny, or my friends would find funny.

Similarly, an autistic participant had a public account to showcase their photography, and a private account that they used to interact with friends.

\section{Multiple Platforms}

Several participants in each group mentioned using different SNS to connect with different audiences as well as to maintain strict boundaries between their public and private lives. For example, one non-autistic participant mentioned that they used Facebook to stay in touch with their family, and other SNS to interact with their friends: It's kind of like Instagram and Snapchat for my friends, [and] Facebook is for my relatives (NA1). An autistic participant reported using the same strategy:

A4: I just usually use Messenger — like, the other app for Facebook - to talk to my family.

Interviewer: Just your family?

A4: Honestly, just my family. Then my friends like friend me and I'm like, "we already have Instagram. What's the point? This is my private life here on Facebook". Like, Facebook is just keeping in touch with family. That's all Facebook is to me. 


\section{Disappearing Content}

Autistic and non-autistic participants mentioned that they sometimes used the dis-

appearing photos/videos feature on SNS to post content that they did not want to keep on their profile indefinitely. For instance, one autistic participant used Instagram Stories to share photos of themself that they did not feel comfortable posting on their profile due to privacy and self-presentation concerns: If I am going to post my own photo, I normally do it on my Story, because it would just be quick (A1). As described in previous research [47], participants' use of this strategy relied on their trust in their peers to not save or distribute their photos to unintended audiences. As one non-autistic participant explained: [On Snapchat], you can give people pictures about anything. You don't have to worry about it staying for a long time, if you trust that they won't screenshot (NA4).

\section{Passwords and Two-factor Authentication}

Very few participants discussed their use of security tools on SNS. Nevertheless, when asked what strategies they use to keep their personal information safe (see Appendix C: Scenario 5), two non-autistic participants mentioned that they kept their account passwords confidential:

NA5: I wouldn't just send somebody my password.

NA15: I don't show my password to anybody. If I login to someone else's phone, I just log off of the account again. So, yeah.

Additionally, one non-autistic participant used the two-factor authentication feature on Instagram:

NA7: Well, they have the verification thing, where you put in your email and then it notifies you if anyone else has logged into your account from another device. That's one of the things I have.

None of the autistic participants discussed their password protection strategies or use of other security tools on SNS. 


\subsubsection{Negotiating Consent}

Both groups discussed negotiating consent with their friends in relation to posting photos on SNS. In line with previous research [47], participants appeared to have a mutual understanding with their friends about what personal content should and should not be posted of each other online. A few participants per group mentioned that they established (often unspoken) ground rules with their friends with regards to sharing photos of each other on SNS:

NA7: All my friends, we know we're not gonna post embarrassing pictures of each other online, 'cause that's not cool.

A5: Most of [my friends] are like me and they don't like having their picture taken. We're all kind of introverts.

All participants trusted that that their friends would not post a photo of them against their consent. Indeed, many autistic and non-autistic participants said that their friends usually asked for permission before posting photos of them on SNS:

NA6: Usually, my friends, if we take a picture together, they would usually ask if they could use it.

A3: People ask me before they post [photos of me.]

Many non-autistic (but no autistic) participants said that they returned the favour:

NA11: Pictures of my friends, I ask them before posting something if they're okay with it. Most of them are.

NA13: I check with the people that are in the picture [...] Just kind of to make sure that they're comfortable being on my account. Even though it's private, you know, someone could still kind of see it, 'cause I do have people that are following me.

On the rare occasion when their friends posted a photo of them that they did not want online, participants in both groups were comfortable asking their friends to remove it. When asked what they would do if their friend posted an embarrassing 
photo of them online, all autistic participants and many non-autistic participants said that they would ask their friend to remove the photo, and expected their friend to grant their request:

NA2: If my friends [post an embarrassing picture of me], I'd just ask them not to do it 'cause they'll understand that I don't want it on there. And they'll just take it down.

A2: [I would] just comment, "can you put it down, please? Because it's kind of embarrassing me. So, it's kinda rude to... May you please put it down?"

\subsubsection{Filtering Out Personal Information}

Finally, both groups protected their privacy on SNS by limiting the type and amount of personal information on their profile. Overall, both samples appeared to have a similar concept of what type of personal information is risky to post on their profile. Most autistic and some non-autistic participants were particularly cautious about posting photos of themselves or their friends online:

NA10: There's no pictures of my face or my friends.

A10: I don't know who I can trust [...] So, I just don't post pictures of myself.

Most participants were also wary about disclosing their current location and address on their profile:

NA3: As humans, we are very repetitive creatures, so we go to the same spots. So, it can be kind of dangerous [to post your location] in that sense.

A11: I don't post anything with a location ever on it.

Other information that many participants in both samples concealed included their full name, age, and contact information.

Non-autistic participants were confident in their ability to determine what type of information is safe and appropriate to post online. Indeed, many non-autistic 
participants were completely certain that they would never post anything on SNS that could harm their safety or reputation: I don't really post anything online that I would think I would regret later (NA4). Realistically, however, it is more than likely that these participants may regret some of their posts later in life. Indeed, Stern [110] found that 40 percent of the adults in their sample regretted content that they posted when they were younger. None of the autistic participants commented on their ability (or lack thereof) to discern what is safe or unsafe to post on SNS.

\subsection{Factors Affecting Privacy Attitudes and Behaviours}

According to our thematic analysis, both groups' privacy attitudes and behaviours were affected by several personal, social, contextual and SNS design factors. Our definition of each factor is presented in Table 4.1. 
Table 4.1: Definition of factors affecting privacy attitudes and behaviours

\begin{tabular}{ll}
\hline Theme & Definition \\
\hline Personal Factors & Impact of users' personal characteristics on privacy attitudes and behaviours \\
Sociability & Interest in interacting with others; agreeableness \\
Social anxiety & Fear of negative evaluation; shyness \\
Previous experience with privacy violations & Privacy violations experienced in the past \\
Gender & Gender differences in privacy attitudes and behaviours \\
Age & Age differences in privacy attitudes and behaviours \\
\hline Social Factors & Impact of other people on privacy attitudes and behaviours \\
Support & Advice or assistance from other people related to privacy protection on SNS \\
Influence & Indirect or unintentional impact of others on privacy attitudes and behaviours \\
\hline Contextual Factors & Impact of the disclosure context on privacy attitudes and behaviours \\
Content & Type of content being disclosed \\
Audience & Who can see users' personal information \\
\hline SNS Design Factors & Impact of the SNS design on privacy attitudes and behaviours \\
Usability of privacy settings & Usability of the privacy settings on SNS \\
General Usability & Usability of the SNS in general, not including privacy settings \\
\hline
\end{tabular}




\subsubsection{Personal Factors}

We identified five main personal factors that influenced participants' privacy attitudes and behaviours: (1) sociability, (2) social anxiety, (3) previous experience with privacy violations, (4) gender, and (5) age.

\section{Sociability}

Autistic participants were generally less sociable on SNS than non-autistic participants. Non-autistic participants viewed SNS as a useful tool to talk to people they know offline: it's a good source to communicate with your friends (NA10). Nearly all non-autistic participants frequently used SNS to send direct messages to their friends. In contrast, most autistic participants rarely or never used SNS to directly communicate with peers. Indeed, several autistic participants described their disinterest in engaging in conversations with their friends online:

A4: I don't talk to them. I don't use the talking features. I just post [to let them know] "this is what I'm doing with my life. Don't talk to me, 'cause I don't really care".

A1: Sometimes I opt out of the conversation because everyone's like " $L O L$ ", "OMG", "LMAO".

Most autistic participants primarily used the direct messaging features for practical purposes, such as making plans with friends and working on group school projects.

A quarter of non-autistic participants reported having an online-only friend who they met through one of the SNS included in this study. Most of these participants talked to their online friends about personal topics, such as school and hobbies, through direct messages.

Interestingly, a greater proportion of autistic participants reported having onlineonly friends than non-autistic participants. However, none of these online friendships were formed on Twitter, Instagram, or Facebook. In fact, all of the autistic participants in our sample met their online friends through gaming-related platforms, such as Discord or Twitch. Although these participants revealed some personal details 
(e.g., first name, nationality) to their online friends, most they were hesitant to discuss their personal life with people they do not know online: There's a wall. You can't pass the wall. Like, people are like "yo, when's your birthday?". And I'm like "you hit the wall" (A4). In many cases, autistic participants exclusively talked to their online friends about common interests (e.g., video games).

None of the autistic participants were motivated to make friends through the SNS examined in this study. Indeed, several participants explicitly indicated that they were not interested in getting to know their followers:

A10: I just don't feel the need to talk to them.

A11: These [random followers] are people that I'll probably never meet and actually have a real conversation with.

In general, autistic participants rigidly classified different users on SNS as either offline friends, acquaintances, or strangers. The very few who occasionally interacted with strangers online were hesitant to call these users their "friends" (even after weeks of frequent communication), instead referring to them as "acquaintances":

A1: I wouldn't call someone that I just met online or seen for the first time online as a friend. I would more just call them an online acquaintance.

A4: If I talk to them enough - like, more than a month's worth of talking - maybe I'll call them acquaintances. But if they're just followers, I don't really care. They're just people.

Non-autistic participants also did not automatically label their unknown followers as friends: I don't know [some of my followers] personally, so I can't say that they're my friends (NA10). However, none of the non-autistic participants mentioned an intermediary ("acquaintance") relationship phase between "strangers" and "friends", labelling all users who they met on SNS and frequently communicated with as their "online friends". These findings suggest that autistic users are much more guarded in online social situations than non-autistic users, thereby decreasing their chances of forming strong and authentic relationships on SNS. 
Additionally, autistic participants adhered to different values and social protocols on SNS than non-autistic participants. Most non-autistic participants were at least somewhat motivated to increase their social capital and status on SNS, using a variety of complex social psychology strategies to attain their goals. For example, one nonautistic participant "randomly" (NA5) followed back most accounts in hopes that this reciprocation will increase their chances of maintaining their follower count. Autistic participants, on the other hand, did not actively try to acquire more followers on SNS. In fact, many of them were fully content with being relatively invisible online:

A8: Nothing really comes up about me, so that's good.

A11: I don't need followers. I have my friends that I talk to at school.

In turn, autistic participants did not take any measures to persuade others to follow their account, such as following accounts back. They carefully curated their followers list, only adding users who posted content they were genuinely interested in viewing. As one autistic participant explained:

A12: I don't really like the whole "follow-for-follow" thing. I just kind of feel like people just want followers and they don't really care who you are. For me, followers are for people you actually look up to or are friends with. I follow a bunch of people because I like them. I think they're cool. Then I also follow friends.

Nevertheless, one autistic participant described that they felt pressured to conform to the non-autistic social values and protocols encouraged by SNS:

A4: I don't want more followers [...] But at the same time, I want more followers. It's like Instagram is trying to trick you into staying on Instagram. Its like the more followers you have, the more people you want to like live up to. It's like "oh, they're following me. That means I have to like post more" and stuff like that. It's just weird.

Autistic participants' unbridled honesty and atypical social conduct occasionally triggered conflicts on SNS. Some autistic participants mentioned posting content that 
may be perceived negatively by others. For example, one participant admitted that they sometimes complained about their parents on Facebook: I'm like, "mom's being annoying"(A4). Conversely, non-autistic participants consciously avoided upsetting others on SNS: Well, obviously you'd take in the consideration of who it might offend. Because nowadays, you can't really go around [posting controversial content] without receiving severe backlash (NA11). It is worth emphasizing, however, that autistic participants usually did not post controversial content with malicious intent. In many cases, it was clear that they did not realize how others may react to their posts or comments. For example, one autistic participant described an incident when they were asked to remove an offensive post that they genuinely thought was harmless:

A1: When I posted a picture of my brother's room, I was like, "why doesn't he clean up"? I wanted to hear everyone else's opinion. And then my mom was like, "that's inappropriate. Do not post that". So, I simply deleted the post. No one has ever seen it since.

\section{Social Anxiety}

Autistic participants were more socially anxious on SNS than their non-autistic counterparts. Generally, non-autistic participants were not worried about being teased by other users on SNS. When asked about their reaction to the scenario where the character's friend posted an embarrassing photo of her online (see Appendix C: Scenario 3), many non-autistic participants were unconcerned about the potential negative social consequences of this situation: You might not want the picture up, but it's not that big of an issue if it was public (NA8). In fact, a quarter of non-autistic participants said that their friends sometimes posted embarrassing photos of them on SNS (and vice versa): What me and my friends do, we post embarrassing pictures of each other on birthdays (NA1). These participants did not mind it when their friends shared these types of photos, recognizing that they were doing it as a harmless joke: If it's a funny picture, I know my friends are just doing it to be a good laugh. And I have a good sense of humor about it (NA3). Although the participants were self-conscious about their physical appearance in these photos, they usually allowed their friends to keep this content online. 
All autistic participants, on the other hand, were against the idea of allowing others to post embarrassing photos of them on SNS. In fact, more than three-quarters autistic participants (versus less than half of non-autistic) did not want any photos of themselves on their profile. When asked why they did not want photos of themselves online, several autistic participants admitted that they were self-conscious about their physical appearance and were afraid of being judged by other users:

\section{A1: I am self-aware of my own appearance and basically how judgmental the rest of the world could be. \\ A11: I don't really let people take pictures of me [...] 'cause people can be rude.}

One autistic participant described an incident where they deleted all of the photos of themself on their profile posted due to self-esteem issues: I once just deleted every picture that had my face in it or body, 'cause I was just having a bad self-esteem moment and so I'm like "delete, delete, delete, delete" (A4). Moving forward, they only posted photos with filters that hid their physical imperfections: The only ones that are there are the ones that have Snapchat filters, so they look a bit better.

Additionally, some autistic participants were concerned about their classmates viewing their online profile because they were afraid of being teased. For instance, one autistic participant explained that they were being bullied at school: Some of the people at school do make fun of me [...] I've heard from my friend saying that people have been saying stuff about me (A11). Although they never experienced any bullying on SNS, they worried that their offline enemies may be laughing at the content on their public profile behind their back: It's kind of uncomfortable, because they can be making fun of me maybe (A11).

Furthermore, autistic participants were much more anxious about initiating contact with people they do not know online than non-autistic participants. Some participants mentioned that they were shy to talk strangers on SNS: I'm pretty shy when talking to people online. But, like, my friends I'm fine. But other than that, I don't usually (A5). None of the non-autistic participants described themselves as too shy to approach or interact with people they do not know online. 


\section{Previous Experience with Privacy Violations}

The most common privacy violations that participants experienced on SNS were unsolicited contact, cyberbullying, and non-consensual disclosure. Although both autistic and non-autistic participants encountered these problems, some violations were more prevalent in one group than the other.

Unsolicited contact appeared to be slightly more common for non-autistic participants. A quarter of non-autistic participants (versus less than a fifth of autistic) reported receiving unsolicited romantic/sexual direct messages or spam from people they do not know on SNS. For example, one female non-autistic participant was sent unwanted romantic messages from older men on Instagram: I've had older guys who are like in their 20 s or 30 s try and almost make marriage proposals to me. And so, I'm like, "yeah, no. This is not happening" (NA7). Similarly, a male autistic participant frequently received inappropriate comments and messages from strangers on Facebook, despite the fact that he had a private account:

A2: See, if [another user] finds your picture a nice picture, maybe something wrong could happen with it. Maybe bad comments, or I don't know.

Interviewer: Has this happened to you before?

A2: Bad comments? A lot. Weird comments, often.

Interviewer: What kind of comments do you mean?

A2: Inappropriate, [from] random people.

Cyberbullying, on the other hand, was substantially more prevalent among autistic than non-autistic participants. A quarter of autistic participants (versus less than a tenth of non-autistic) described being bullied by people they do not know online. One autistic participant explained that they had to block some users on Instagram who were bullying them on another social media platform: There's a lot of people who harass [me] from other social media who have found [my Instagram profile]. I'm like "stay away! I'm blocking you. Go away!" (A4). Another autistic participant mentioned that they did not talk to people they do not know on Instagram and had set their profile to private due to a negative experience interacting with strangers on 
another SNS platform several years ago. Only one non-autistic participant mentioned previously being involved in conflict on SNS:

NA7: There have been people — like, I comment on someone else's post and then got into a fight with them online because they don't know the full story or they're making up things. And I just don't want to deal with that, so I just kind of block them.

Additionally, a few autistic participants reported having photos of them posted on SNS without their consent. Two autistic participants said that they had to report or ask their friend to remove a photo of them "once or twice" in the past. Although most non-autistic participants were unconcerned about the photos that their friends posted of them on SNS, a few female participants described a situation in which their friends shared an unflattering "selfie" that they did not want online: There was this one picture that I didn't really like that I looked very ugly, but [my friend] took it down when I told her to (NA16). This finding is consistent with teenagers' typical reliance on "retroactive consent", discussed in previous research [47].

Furthermore, one autistic participant mentioned encountering a potential identityrelated privacy risk on SNS: they were mislead into believing that one of their onlineonly friends on Discord was older than they actually were:

A4: My [online] friend, he used to use a voice changer and [...] he'd start talking to me in that voice at the beginning [...] I was [on Discord] for like a month, so I didn't like catch on properly. But all my other friends were like, "yo, you know this kid's like 13, right?". I'm like "oh, he sounds like he's 16. This is so weird".

\section{Gender}

In previous research, gender has been identified as a main factor influencing teenagers' privacy attitudes and behaviours on SNS $[32,100,118]$. However, our analyses revealed few notable differences between male and female participants in either of our samples. The only noticeable gender difference that we found related to social anxiety. In general, female participants tended to express more concerns about their physical 
appearance and self-presentation on SNS than male participants. In turn, female participants were more likely to edit or remove photos of themselves on SNS than their male counterparts.

Furthermore, we found some evidence that non-binary users may be more open about disclosing certain aspects of their offline identity on SNS than males or females. One of the autistic participants in our sample identified as non-binary and queer. For this participant, the anonymity of the online environment provided them the comfort to discuss and explore their identity:

A4: My sexuality and gender, I keep it online. I have too many selfesteem problems [offline]. I only put it online because I trust people online for some reason. The people online are more caring sometimes.

\section{Age}

We analyzed our data for differences between the privacy attitudes and behaviours of younger (13 to 14 years) and older (15 to 17 years) participants, respectively.

Consistent with previous research $[18,27]$, age appeared to be a main factor impacting non-autistic participants' risk-taking behaviours and, consequently, their privacy and safety on SNS. Older non-autistic participants were more likely to have a public profile than younger non-autistic participants. They also were more open to posting pictures of themselves and talking to people they do not know on SNS than their younger counterparts. These trends may be partly due to the higher parental support and influence that younger non-autistic participants had compared to older users. More specifically, younger non-autistic participants used SNS less actively than older non-autistic participants because their parents regulated their screen time and monitored their account.

Only three of the autistic participants in our sample were below 15 years old; therefore, we were unable to examine age-related differences in the autistic group. However, we noticed that our autistic participants (who were mostly older teenagers) tended to have similar privacy attitudes and behaviours on SNS as younger nonautistic participants. Few autistic participants posted photos of themselves on their profile or talked to strangers online. Furthermore, as will be discussed in the next 
section, autistic participants' SNS use and privacy-protective behaviors were highly influenced by their parents' involvement in their online life.

\subsubsection{Social Factors}

Our thematic analysis revealed two main social factors affecting participants' privacy attitudes and behaviours on SNS: (1) support and (2) influence.

\section{Support}

Both autistic and non-autistic participants received support from teachers, family members and friends to protect their privacy on SNS. Teachers and parents played a crucial role in directly educating both groups of participants about the privacy risks of SNS use and how to mitigate them. When asked what their parents and teachers (respectively) taught them about online privacy, most autistic and non-autistic said that they were told to be careful about what they post on SNS:

NA11: [My father] told me to in general think about would you be comfortable if this was online. Would you be comfortable with this being there forever? Because if you post something that you're happy about it at the moment, you might not be so proud or happy given 2 to 3 years. And even if you do take the post down, there's the chance that somebody somewhere has saved that. So, now it's in their hands.

A11: My mom said when I was younger that you're not supposed to show your face on like the Internet, 'cause some people can use your picture to catfish other people and stuff.

Similarly, participants in both groups were instructed not to talk to strangers on SNS to protect their safety:

NA7: My mom told me, "don't talk to anybody that you don't know, and don't talk to anyone that's, you know, like... I guess weird."

A1: Everyone that I know have constantly told me don't talk to people you don't know online because of safety reasons. They may try to bribe you to 
show a picture of yourself or saying something that they can use against you - anything really.

Some said that they primarily learned about cyberbullying in school:

Interviewer: What did school teach you about online privacy?

NA2: Just how to prevent bullying.

A10: Mainly just cyberbullying. Yeah, that's pretty much the only thing.

In terms of learning about the privacy settings on SNS, autistic and non-autistic participants typically relied on informal instruction provided by their family members and friends:

NA13: Well, I got Instagram not that long ago when I went to school for 7th grade. So, there was already people that kind of knew Instagram, 'cause they had it for a while. I think they told me about [the private account option].

A1: At the time [I started using Instagram], I didn't know how Instagram necessarily worked. I didn't know where to find [the private account option]. All of my friends were like "it's right here".

Although both autistic and non-autistic participants received similar advice about protecting their privacy on SNS, there were some notable differences between the two groups in terms of the amount and type of support they received. For instance, autistic participants received more active assistance to use privacy settings than nonautistic participants. Non-autistic participants described that their parents or friends verbally instructed them how to set their privacy settings in their preferred way: I knew that you can make your account private, but I really didn't know at the beginning [how to do it]. [My friend] taught me that you just go here, click on that, and then you can make your account private (NA9). Only one non-autistic participant said that someone else had set their account to private for them. In contrast, a third of autistic participants indicated that their friends or parents tried to show them how to adjust their privacy settings by taking hold of their device/account and doing the steps for them: 
A4: My friends, they just snagged the phone and they're like "this is what you do".

A10: [My mom] set it to private and then after that she said it was in the settings.

In some cases, this low autonomy lead to distress. Indeed, two autistic participants said that they were upset that their device (i.e., smartphone) was taken away from them when their friends were showing them how to adjust their privacy settings. One participant explained that their phone served as an important tool to help them manage their autism symptoms:

A4: It helps with my autism, my phone [...] It's like being able to call my mom if I'm having an attack or something. And also music helps calm me down, so it's like quick access to music and distractions. It just helps me distract myself.

Therefore, they felt anxious when they did not have direct access or control over their device while their friends were changing their privacy settings for them.

Additionally, only autistic participants reported receiving support to use the general features on SNS. One participant mentioned that their mom helped them to post pictures on Facebook. Their friends also taught them how to adjust the volume settings on Discord. Another participant reported that their sister helped them to use the search feature on Instagram.

\section{Influence}

Both groups' privacy attitudes and behaviours were influenced by those around them. As expected, non-autistic participants' friends had the greatest influence on their privacy concerns and protective strategies on SNS. Several non-autistic participants said that they learned about the privacy risks of SNS use and how to respond to them from their friends' experiences:

NA3 (female): I have a lot of friends who get pictures from random guys. So, you hear what they do, and you take that in, and consider if it happens to me, what do I have to do? Then you kind of just learn from there. 
NA11: I've learned from my friends' experiences - the ones who've made mistakes on social media sharing private information. And in no case has it ended well for them. [They shared] private information as in something more intense than what you would usually post [...] It was pictures of them with minimal clothing. You don't want to be sending those out at 13 or 12 years old, because it's illegal!

Additionally, a few non-autistic participants said that they followed their friends' lead in setting their profile to private: I asked [my friends], "why is [your Instagram profile] privated"? And they were like, "just to protect it, I guess". I was like, "might as well do that to myself, right?" (NA2). Moreover, some non-autistic participants said that their decision to sign up for SNS and their choice of platform was inspired by their friends' preferences: I'd say [I signed up for Instagram] a month and a half ago, because all of my friends had Instagram (NA12).

Although many autistic participants were similarly influenced by their peers, their parents appeared to have the greatest impact on their privacy attitudes and behaviors on SNS. Several autistic participants made their account private to minimize their parents' concerns about their safety online. For instance, when asked why they have a private account, one autistic participant explained: My mom just feels more comfortable with me having a private account (A8). Also, a few autistic participants said that their parents influenced their use of SNS. In some cases, parents seemed to promote autistic participants' engagement in online social networking. For instance, one autistic participant reported that they personally do not enjoy using Facebook; however, their mom (who they described as a frequent Facebook user) encouraged them to use the site. Other participants said that their parents attempted to limit the amount of time that they spend on SNS:

Interviewer: When do you use Instagram?

A9: When my mom says I could.

Furthermore, some autistic participants mentioned that their parents monitored their account; in turn, they refrained from sharing content that their parents told them 
not to post. In the non-autistic group, such examples of parental influence were only reported by a few younger non-autistic participants.

\subsubsection{Contextual Factors}

Two main contextual factors impacted autistic and non-autistic participants' privacy attitudes and behaviours on SNS: (1) content and (2) audience.

\section{Content}

Both groups' privacy behaviors depended on the sensitivity of the personal information intended to be posted on SNS. All participants were unconcerned about posting content that reflected their hobbies, interests, and experiences because they did not consider this type of information to be personal or classified. In contrast, most were at least somewhat wary about sharing pictures of themselves or their family, their location, their contact details, and other personal identifying information. Many participants decided to make their profile public because they did not post anything that they considered to be personal. For instance, one non-autistic participant described that they mostly their Twitter account to connect with the feminist community: What I retweet is usually about feminist stuff (NA6). They did not think it was necessary to have a private account, because they did not post about their personal life. Similarly, many autistic participants solely dedicated their account to showcasing their talents and interests (e.g., photography, visual arts, music). Most of these participants allowed strangers to view their content and follow them because they felt relatively anonymous. For example, one autistic Twitter user primarily shared content related to their main interest (i.e., computer programming), and avoided posting personally identifying information:

A12: I mostly either post just responses to people posting other stuff and just programming jokes. I don't really often post pictures at all. I've never posted a picture at all, actually [...] I don't really like sharing my identity on Twitter. I'm okay with sharing personal things, but not like who I am. I'm okay with what I do, but not who I am. 
When asked who they wanted to see their posts, the participant replied: It doesn't really matter to me, to be honest (A12). This lack of concern about their content contributed to their decision to have a public account.

\section{Audience}

Both autistic and non-autistic participants were mindful of who can see their content on their social media profile, and took measures to regulate their online audience based on their personal privacy preferences (discussed in section 4.1). Most participants said that they wanted people they know (e.g., friends, family) to see their posts:

Interviewer: Who do you want to see your pictures?

NA16: Just my very close friends.

A8: Just people I know.

Some participants in both groups were concerned about strangers or people with whom they had negative experiences (e.g., bullies, exes) viewing their profile. Many of these participants had a private account to restrict access to their content.

Other participants were unconcerned about who can see their content:

Interviewer: Who do you want to see your pictures?

NA8: I'm fine with pretty much most people.

A12: I don't really mind if someone sees my posts.

These participants had public accounts.

\subsubsection{Social Networking Site Design Factors}

Both group's privacy attitudes and behaviours were also affected by the usability of the privacy settings and SNS in general. 


\section{Usability of Privacy Settings}

As expected, autistic participants had more difficulty using privacy settings on social media than non-autistic participants. Most non-autistic participants did not require support or training to learn about the privacy options on SNS and how to use them; they acquired this knowledge and skills by simply exploring the interface:

Interviewer: Who taught you how to set your account to private?

A2: Myself. I just learned it while playing more often on the computer. No one taught me that.

NA6: Myself. I was just like browsing, I'd say. Just looking around.

Several participants alluded to the consistency of privacy options across SNS platforms as facilitating their use of these tools. For example, one participant described that they transferred their knowledge of privacy settings on Facebook (i.e., the first SNS they ever used) to later protect their Instagram account:

NA14: When I first got Facebook, my dad told me that my account wasn't private. I didn't know what private or not was. And so then, when I ended up getting Instagram a couple years later, I wanted to make it on private, so that I can control who's looking at my photos.

Many non-autistic participants explained that they expected certain privacy settings (e.g., private account, blocking users) to be available on all SNS: Well, it seems like [the block user option is] a very standard feature to have in something like this because there will always be a case where someone is following you who you don't want to follow (NA11). Most participants also expected that the private account option would always be located in the settings tab on SNS. When asked if their friends helped them to set their account to private, a participant replied: I actually just went into settings and then you can probably figure out from there, right? (NA2). Most non-autistic participants described the privacy settings on SNS as easy to use: It is just kind of an obvious interface (NA5).

In contrast, most autistic participants described the privacy settings on SNS as "complicated". When asked to demonstrate how to set an SNS account to private, 
many autistic participants struggled to accomplish this task, and two participants were unable to do it at all. Many participants had difficulty finding the private account button because it was hidden among many other options in the settings tab: There were tons of settings [...] What is this maze?! (A1). Some participants coped with this challenge by searching for keywords (e.g., "privacy") on the screen: You just go through your settings and then you'll find it. Use keywords. Like [when I see] "privacy", I'm like "yeah, that's gotta be private your account" (A4). This approach did not work for one autistic Twitter user, due to the unconventional labelling of the private account option on their preferred SNS. The participant admitted that they did not know how to set a Twitter account to private, but they volunteered to try to accomplish the task. They quickly deduced that the setting was under the "Privacy and Safety" tab; however, it took them a few minutes to realize that the option they were looking for was labelled "Protect Your Tweets". The participant explained that they had overlooked the option because its wording was not intuitive for them: I glossed over [the "Protect Your Tweets" option] because I really didn't understand what it meant until I read the subtext on it (A12). In response to their experience, the participant said that Twitter should change the labelling of the privacy setting to be more intuitive and consistent with typical conventions: I think they should probably rename [the option] to something like "make your tweets private" [...] I think that they should make it more obvious.

Additionally, autistic participants were less aware of certain privacy options on SNS than non-autistic users, possibly because the settings were not sufficiently featured on the interface. A third of autistic participants (versus less than a tenth of non-autistic) did not know about at least one of the main privacy settings available on all SNS - namely, private account, block user, report user and report content. For instance, when asked what someone can do if their friend refused to remove an embarrassing picture of them on Instagram, the "report inappropriate" option did not come to two autistic participants' minds:

A11: Don't really know if [they] could do much.

A9: If [the] friend refuses [to take down the photo], [they] can't do anything about it. 
In some cases, autistic participants' lack of awareness of the privacy settings discouraged them from posting personal content on their profile. One participant, who did not want people they do not know online to see photos of them, explained:

A10: Before I made my account private, some people followed me who I don't know. [If I block them,] some people might be like "oh, hey, I was just following you to see pictures of your cats". Then, they might be like "well, that's rude. He just blocked me for no reason" [...] It's like, if they want to unfollow me, they can. If not, I'll just let them stay. I just won't post pictures of myself.

In this situation, the participant's behaviours were constrained by social norms related to the "block user" tool on Instagram - a feature that most users perceive as aggressive (as will be discussed later in this section). In turn, the participant adopted an alternative approach to protect their privacy: they asked some of the users who were following them before their account was private to unfollow them:

A10: If I had personal information on my account, I would probably message him saying "hey, I forgot to make my account private. Would you mind unfollowing me, 'cause I don't want to be rude and block you". If they don't, then I already asked them to unfollow me, so then I'll block them if they don't unfollow.

Interviewer: Did you do that before?

A10: Yeah. I did that with one person.

They did not seem to know about Instagram's "remove follower" option, which would do the same thing without having to engage in an awkward social encounter.

Furthermore, more autistic participants misunderstood the privacy settings on SNS than non-autistic participants, providing further evidence of cognitive accessibility issues in the settings' design. A third of autistic participants (versus less than a quarter of non-autistic) had a clearly inaccurate understanding of at least one privacy setting on SNS. One autistic participant believed that the "hide from timeline" option on Facebook would remove any post from the site: If you press this ["hide 
from timeline"] button, you can hide [the post] from you or like anybody else (A2). In reality, this feature only removes posts from appearing on the user's own feed and profile. Another autistic participant thought that SNS profiles were private by default (whereas the opposite is the case); after viewing the video clip where the character asks if the participant knows how to set their account to private (see Appendix C: Scenario 4), the participant replied: That doesn't make any sense because her account should already be private to begin with, so she must have changed it to be public (A9). One autistic participant's misconception of the private account option on Instagram prevented them from using this setting. The participant, who often used Instagram to contact group members for school projects, thought that having a private account would prevent them from sending and receiving messages from people who are not following them and who they are not following back: If I have a private [account], then [people] can't [direct message me] without me following them back (A11). In reality, Instagram allows users to send direct messages to anyone on the platform, including those with private accounts. The participant did not want some of their classmates to be able view their profile because they were afraid of being teased; yet they chose to make their account public to contact people through the site.

Although the usability of the privacy settings differed substantially between the autistic and non-autistic participants, there were some design issues that were equally problematic for both groups. Firstly, both groups thought that some of the privacy settings on SNS - namely, reporting and blocking users or content - are aggressive and would cause unnecessary arguments with friends when used. For instance, most participants said that if their friend posted an embarrassing photo of them online, they would just ask their friend to remove it. Those who were aware of the "report" privacy tool on SNS said that they would only use it if their friend refused to take the photo down. When asked why they would not use the privacy setting right away, participants explained:

NA11: You're not gonna report [the photo] unless you've already told your friend that you don't want it there. I would assume that no one would really kill a friendship over an Instagram post [...] But if you go right ahead and you can ask the person to take it down and in the scenario 
where the person does not choose to take it down, then you can report and you can write a formal letter of complaint and it will be taken down. [But] only as a last resort.

A10: If you just report it right away, they might get mad at you. Like, "why'd you report my photo?". I don't know if it tells the person that you reported their photo, 'cause I haven't done it. But they might get mad at you and say "why'd you report it? You could have just talked to me. You could have just asked me to take it down". Like, you could have avoided them getting mad at you by just asking them.

Additionally, both autistic and non-autistic Instagram users had difficulty knowing whether their friends' accounts were private or public because this information is not currently displayed on users' profiles. Participants realized that they needed to rely on their memory to know their friends' privacy settings. As one participant explained:

A10: Before you followed them, you'd either have to request to follow them or you could just follow them without requesting. So, if you have to request, then it means that their account is private. If you can just see their pictures without following them, and you can follow them without requesting, then it's not private.

Many autistic and non-autistic participants struggled to recall whether they initially requested to follow their friend. One autistic participant explained: Once you start following them, you don't remember that it's locked (A1). Similarly, a non-autistic participant tried to show the interviewer how a private account appears to users who are not following it by pulling up a friend's profile that they believed was private (based on their memory of sending a request to follow the account); they were surprised to realize that their friend's account was not private after all:

NA8: If we go to, let's just say (searches friend's account) ... I think it's private... No, it's public. What the $f^{*} c k$ ? That's weird [...] I'm trying to find one that was private...(searches another friend's account) Damn it! You keep it public too? Why is it...? (sigh) ... Who do I know who has a 
private account? I don't really know. I don't really remember off the top of my head.

The participant later pointed out another issue with not clearly displaying other users' privacy settings on profiles - that is, not knowing when others change their settings: They might have originally been private and then made it public. Autistic and nonautistic participants proposed a few ways of knowing if a friend's account is private or public. When asked how the character in the scenario videos can figure out her friend's privacy settings on Instagram, one non-autistic participant said that she could ask her friend for this information: I guess you could just ask if the account is private or not (NA3). However, they specifically recommended doing so if the other user is a "close enough friend" (NA3), suggesting that privacy settings may be a sensitive topic for teenagers to discuss with their peers. An autistic participant proposed a more creative solution to deducing other users' privacy settings on Instagram:

A10: Well, you could check [the privacy settings] by unfollowing [the friend] and re-sending the follow request. Then, if they ask like "why did you unfollow me", you could just tell them "well, I was just checking if your account is private".

Another participant believed that the technology itself should provide information about other users' privacy settings: [The site] should tell you. Like, there should be something beside somewhere that says "this person is [public or private]" (NA14).

Furthermore, both autistic and non-autistic participants thought that the "report" privacy setting was ineffective. One non-autistic participant said that they tried reporting hate speech on Twitter, but the post was not removed. Based on their experience, they concluded that "the report button doesn't really do much" (NA4). Similarly, an autistic participant who reported a photo on Instagram found the privacy setting unresponsive: I check once in a while, "is [the photo] gone yet?". And if it's not, I report it again until it disappears (A4). 


\section{General Usability}

SNS were less usable in general for autistic participants than non-autistic participants. None of the non-autistic participants reported any substantial difficulties using SNS. Indeed, several non-autistic participants described SNS as "easy" to use, and none required support to navigate through the platforms.

Several autistic participants, on the other hand, had trouble using certain features on SNS. One autistic participant had difficulty learning how to use Facebook Messenger on their own: I'm still trying to learn how to use Messenger. It's hard. It's new (A2). Another autistic participant forgot how to play games on Facebook: I used to play games on there. And then I forgot how to play games so I stopped doing that (A7). They also thought that it required too much effort to post pictures on the site: I try to just take a picture of myself and then have to put it onto my computer or phone and then try to post it (A7). Another autistic participant criticized Twitter for being too text-based and therefore mentally exhausting to use: Twitter is a mess! It's just a mess of words, and you're just like "no thank you". Especially when you're tired, Twitter's the worst! (A4). They described Instagram as more enjoyable to use in their downtime because it requires less reading and concentration than Twitter: Instagram is more simple. It has a better layout than Twitter [...] Instagram, when you're tired, it's just pictures. You can just swipe really quickly and you'll just see stuff (A4). The participant also mentioned that frequent user interface updates made it challenging for them to continue using SNS (especially Twitter) after taking an extended social media break:

A4: [Twitter is] one of the most complicated sites [...] It just updates and it changes a lot. I was off it for like a month and then I just see it again and I'm like "oh god! Everything changed! Even the screen layout and all that". And I was like "ugh!!". It was just really weird.

A few autistic participants also misunderstood some of the features on SNS. For instance, one autistic participant thought that their location was automatically detected and shared with others every time they posted a picture on Instagram: Anytime someone posts, it says where they are - where the post is [...] It's an automatic thing that 
I don't like (A9). In reality, Instagram users must manually enter their location for this information to appear on posts. Due to this misunderstanding, the participant was hesitant to post pictures at home out of concern for their safety.

Overall, non-autistic participants attributed a high value to SNS. A few nonautistic participants said that SNS have content and features that are relevant to their interests: It's got a lot of stuff for like stuff that I like on it (NA13). More notably, non-autistic participants emphasized the usefulness of SNS to contact their friends: If I want to hang out with someone, Instagram is just that platform you could easily talk to them (NA3).

Autistic participants had more mixed opinions about SNS. A few autistic participants genuinely enjoyed using social media. They thought that certain features (e.g., voice/face chat, face filters) were fun to use:

A2: Face chat is actually interesting. They see your face on the thing.

A4: The face filters are pretty cute! I think it's just funny to see cute videos, like people voice changing. I just like it. It just makes me laugh.

Some autistic participants also said that SNS are useful for staying up-to-date with their interests: I just kind of look at other people's stuff. It's really fun (A8). A few participants said that SNS are helpful for staying in touch with friends who moved away: [My friend] had moved away and I was never going to see them again. And so, I make sure to follow them (A1). Nevertheless, autistic participants placed less value on SNS than their non-autistic counterparts. One autistic participant said that they rarely used Facebook because they did not find it entertaining: I just don't like using Facebook [...] I just don't find it fun (A7). They only used it to view what their mom posts. Another participant said that they got bored of the Explore feature on Instagram shortly after discovering it: I looked at it and I'm like "I'm bored". Then I put it down (A4). Overall, it was clear that autistic participants prioritized other interests over using SNS. One participant explained that they stopped posting photos on Instagram, believing that they had better things to do with their time:

A8: I don't really use Instagram to post pictures of myself. I just kind of browse around and look at other people's stuff. But that's been lately. 
When I first got it, I used to do pictures all of the time. I just have a lot on my hands right now. [Posting pictures takes] up time, and it's just not something you need.

Similarly, another autistic participant forgot that they had a Twitter account because the site was not engaging enough to be incorporated into their daily routine:

A12: I used to use [Twitter] a lot more actually. But I've kind of stopped using it recently.

Interviewer: Why?

A12: Just habit, I guess. I just kind of forgot that I had the account.

\subsection{Summary}

Tables 4.2 and 4.3 summarize our findings. Overall, we found several key differences and similarities in autistic and non-autistic participants' privacy attitudes and behaviours on SNS. We also identified privacy-related factors that affected one group more than the other.

\subsubsection{Privacy Attitudes}

Contrary to expectations, the autistic participants in our study were not more "naive" to privacy risks on SNS than their non-autistic counterparts. In fact, they were highly conscious of extreme privacy and safety threats online, and were very concerned about falling victim to them. Unlike non-autistic participants - most of whom attempted to balance privacy and self-disclosure on SNS - almost all autistic participants prioritized their online privacy and safety over active engagement on SNS.

\subsubsection{Privacy Behaviours}

Autistic and non-autistic participants used similar strategies to protect their privacy on SNS. These strategies included (1) deciding who to trust, (2) managing access, (3) negotiating consent, and (4) filtering out personal information. Although both groups engaged in the same privacy-protective behaviours, autistic participants used 
more strategies to prevent unauthorized access to their personal information than non-autistic participants.

\subsubsection{Privacy-Related Factors}

Both groups' privacy attitudes and behaviours were affected by several personal, social, contextual, and SNS design factors.

\section{Personal}

As anticipated, autistic participants' unique disposition partially accounted for the differences between the groups' privacy-related thoughts and actions. In general, autistic participants were very guarded and socially anxious on SNS, expressing high discomfort with initiating contact and having intimate conversations with people they do not know online. In contrast, non-autistic participants - particularly the older ones - were motivated to increase their social capital and were open to discussing their personal life with people they met on SNS. Autistic participants' low sociability sometimes caused conflict. Indeed, autistic participants were more likely to commit social blunders on SNS than their typically developing counterparts. However, it was clear that autistic participants did not intend to offend people; they did not know how others would react to their posts. This may be due to autistic participants' limitations in social cognition, such as theory of mind [11].

\section{Social}

Parents, teachers and peers supported and influenced participants' privacy attitudes and behaviours. As expected, autistic participants required more social support to use privacy settings and other features on SNS than non-autistic participants. In some cases, this reduced autonomy led to distress, highlighting the importance of developing accessible privacy tools for this user population. Unlike non-autistic participants whose online activities and privacy were mainly influenced by their peers - autistic participants' use of SNS and online safety were mostly directly and indirectly affected by their parents, who appeared to vary in their mediation strategies. 


\section{Contextual}

Unsurprisingly, participants' privacy attitudes and behaviours varied depending on sensitivity of the content they intend to disclose and the anticipated audience. Overall, both groups were relatively comfortable disclosing content related to their interests, hobbies and experiences, and cautious about sharing personally identifying information. Autistic participants, in particular, preferred to be anonymous online, as discussed in previous research $[12,20,89]$. Although we found individual differences in participants' privacy preferences, both groups primarily wanted their friends and family to view their posts.

\section{Social Networking Site Design Factors}

As predicted, autistic participants had much more difficulty using privacy settings and SNS in general than non-autistic participants. Whereas non-autistic participants described privacy settings and SNS as "easy" to use, autistic participants found the interface "complicated" and unintuitive. In many cases, accessibility issues with the design of SNS prevented autistic participants from using privacy settings and discouraged them from actively and continuously posting on the platforms. 
Table 4.2: Summary of findings: privacy attitudes, privacy behaviours, per-

sonal factors

\begin{tabular}{|c|c|c|}
\hline Theme & Non-Autistic & Autistic \\
\hline \multicolumn{3}{|l|}{ Privacy Attitudes } \\
\hline & $\begin{array}{l}\text { - Only aware of social privacy threats } \\
\text { - Low or moderate privacy concern } \\
\text { - Somewhat wary of strangers } \\
\text { - High trust in peers } \\
\text { - Balance privacy and self-disclosure }\end{array}$ & $\begin{array}{l}\text { - Most only aware of social privacy threats } \\
\text { - High privacy concern } \\
\text { - Extremely wary of strangers } \\
\text { - Moderate trust in peers } \\
\text { - Prioritize privacy over self-disclosure }\end{array}$ \\
\hline \multicolumn{3}{|l|}{ Privacy Behaviours } \\
\hline & $\begin{array}{l}\text { - Use four main strategies:(1) deciding who to trust, (2) managing } \\
\text { access, (3) negotiating content, (4) filtering out personal informa- } \\
\text { tion }\end{array}$ & - Same strategies as non-autistic, but use them more \\
\hline \multicolumn{3}{|l|}{ Personal Factors } \\
\hline Sociability & $\begin{array}{l}\text { - Frequently communicate with friends } \\
\text { - Have online friends who they met on SNS; discuss personal life and } \\
\text { common interests } \\
\text { - Use complex social protocols to build social capital } \\
\text { - Careful not to offend others }\end{array}$ & $\begin{array}{l}\text { - Rarely use direct messaging feature } \\
\text { - Have online friends (or 'acquaintances') who they met on gaming } \\
\text { platforms; only discuss common interest } \\
\text { - Do not actively try to build social capital } \\
\text { - Accidentally cause conflict }\end{array}$ \\
\hline Social Anxiety & - Low fear of negative evaluation & $\begin{array}{l}\text { - High fear of negative evaluation } \\
\text { - Shy }\end{array}$ \\
\hline $\begin{array}{l}\text { Previous Experience } \\
\text { with Privacy Violations }\end{array}$ & - Unsolicited contact was most common & - Cyberbullying was most common \\
\hline Gender & - Higher social anxiety among females than males & $\begin{array}{l}\text { - Higher social anxiety amongh females than males and non-binary } \\
\text { - Greater self-disclosure by non-binary than males and females }\end{array}$ \\
\hline Age & $\begin{array}{l}\text { - Greater risk-taking among older teens than younger } \\
\text { - Lower parental support and influence among older teens than } \\
\text { younger }\end{array}$ & $\begin{array}{l}\text { - Similar privacy attitudes and behaviours as younger non-autistic } \\
\text { teenagers }\end{array}$ \\
\hline
\end{tabular}


Table 4.3: Summary of findings: social factors, contextual factors, SNS design factors

\begin{tabular}{|c|c|c|}
\hline Theme & Non-Autistic & Autistic \\
\hline $\begin{array}{l}\text { Social Factors } \\
\text { Support }\end{array}$ & $\begin{array}{l}\text { - Formal and informal privacy education } \\
\text { - Little support required to use privacy settings and other features }\end{array}$ & $\begin{array}{l}\text { - Formal and informal privacy education } \\
\text { - Require active assistance to use privacy settings; reduced auton- } \\
\text { omy causes distress }\end{array}$ \\
\hline Influence & - Peers have greatest influence over privacy attitudes and behaviours & $\begin{array}{l}\text { - Parents have greatest influence over privacy attitudes and be- } \\
\text { haviours }\end{array}$ \\
\hline $\begin{array}{l}\text { Contextual Fact } \\
\text { Content }\end{array}$ & $\begin{array}{l}\text { - Comfortable disclosing interests, hobbies, and experiences } \\
\text { - Wary of posting personally identifying information }\end{array}$ & - Same as non-autistic \\
\hline Audience & - Primarily want friends and family to see posts & - Same as non-autistic \\
\hline $\begin{array}{l}\text { SNS Design Fac } \\
\text { Usability of } \\
\text { Privacy Settings }\end{array}$ & $\begin{array}{l}\text { - Privacy settings are "easy" to use } \\
\text { - Reporting and blocking tools viewed as aggressive and ineffective } \\
\text { - Difficulty knowing other users' privacy settings }\end{array}$ & $\begin{array}{l}\text { - Privacy settings are "complicated" } \\
\text { - Less aware of privacy settings than non-autistic } \\
\text { - Greater misunderstanding of privacy settings than non-autistic } \\
\text { - Reporting and blocking tools viewed as aggressive and ineffective } \\
\text { - Difficulty knowing ohter users' privacy settings }\end{array}$ \\
\hline General Usability & $\begin{array}{l}\text { - No substantial usability issues } \\
\text { - Highly value SNS }\end{array}$ & $\begin{array}{l}\text { - Substantial difficulties using SNS } \\
\text { - Prioritize other hobbies over using SNS }\end{array}$ \\
\hline
\end{tabular}




\section{Chapter 5}

\section{Discussion and Conclusion}

The objectives of this study were to compare the privacy attitudes and behaviours of autistic and non-autistic teenagers on SNS, and identify factors affecting these users' privacy and safety in online social contexts. Specifically, we investigated the following research questions:

RQ1: What are autistic teenagers' thoughts and feelings about their privacy on SNS, and how do they protect their online privacy?;

RQ2: What are the main factors affecting autistic teenagers' privacy attitudes and behaviors on SNS?; and

RQ3: How do these privacy-related attitudes, behaviours, and factors compare with those reported by non-autistic teenagers?

To answer our research questions, we conducted scenario-based, semi-structured interviews with teenage social media users with and without autism about their SNS use, privacy concerns, and strategies for mitigating online risks. In this chapter, we discuss the lessons learned from our research, and provide recommendations for maximizing the benefits of online social networking for all teenagers. We conclude by acknowledging the limitations of our study and proposing avenues for future research.

\subsection{Lessons Learned}

To our knowledge, this is the first study to examine autistic teenagers' privacy attitudes and behaviours on SNS, and to compare these users' perceptions of and experiences with online risks to the general population. Therefore, our research provides novel insight into the role of neurodiversity on SNS users' online privacy and safety. In the following sections, we detail the relevance and contributions of our findings to the broader literature on teenagers' online privacy and social networking, as well as on cognitive accessibility and on inclusive research. 


\subsubsection{Autistic Users' Vulnerability to Online Risks: Exposing Miscon- ceptions through Inclusive Research}

Autism is often linked to high social vulnerability [35, 45]. Indeed, several studies show that autistic people are more at risk of abuse, exploitation, and victimization than the general population in real life $[35,45,102]$. With the increasing ubiquity of online social networking, there has been growing concerns regarding autistic people's vulnerability to privacy and safety threats - especially cyberbullying and sexual solicitation — on SNS [39,48,50,130]. Previous studies describe autistic people as exceptionally "naive" to Internet risks, as well as "obsessive" and "impulsive" when engaging in online activities $[39,48]$. However, there are notable limitations with existing research in this area. Firstly, most studies exclusively shed light on experts' and caregivers' perspectives on the matter or employ cognitively inaccessible data collection methods (e.g., surveys) that exclude the perspectives of autistic people with intellectual disabilities. Secondly, the majority of studies do not actually compare autistic and non-autistic users' experiences on SNS, despite making claims about differences between the two groups. Finally, some studies assume that autistic users' purportedly reckless behaviour on SNS is due to their social-cognitive impairments and/or intellectual disabilities; they do not acknowledge other factors that may be constraining these users' knowledge and use of effective strategies to prevent and cope with negative online experiences. These limitations contribute to misconceptions about autistic users' privacy-related thoughts and protective behaviours on SNS that we expose through our inclusive research.

Contrary to caregivers' and experts' perceptions [39,48], we found that the autistic teenagers in our study were not more oblivious to social privacy risks online than the general population. In fact, autistic teenagers appeared to be equally aware of predators and the potential for harassment on SNS as their typically developing counterparts. This finding suggests that autistic users are in fact capable of developing online privacy literacy, if offered the necessary support and resources. Therefore, efforts should be made to enhance online privacy education for this population.

We also observed notable differences in the two groups' privacy perceptions and behaviours on SNS that contradict popular beliefs about autistic users' vulnerability 
to online risks. For instance, researchers postulate that autistic users are more inclined to engage in risky behaviours on SNS, such as making friends with people they meet online, than non-autistic users to reduce their loneliness and receive social support [39]. In other words, experts believe that autistic users are more likely to trade their privacy and safety on SNS for social benefits than the general population. In our study, however, we found that this is not the case. Unlike non-autistic teenagers, who normally strive to balance privacy and sociability on SNS, autistic teenagers tend to be exceptionally preoccupied with protecting their privacy and safety online due to extreme concerns. They are also usually too shy or not interested in selfdisclosing to people they encounter on the Internet. Although a greater proportion of autistic teenagers in our study had online friends or "acquaintances" than the nonautistic group (in line with previous research [38]), their conversations with strangers on social media almost exclusively focused on common interests and very rarely lead to risky self-disclosures. Overall, autistic teenagers used more strategies to protect their personal information and identity on SNS than their typically developing peers, making them safer to many (although not all) online risks than the general population. These findings call to question previous assumptions about autistic teenagers' propensity towards risk-taking in online social contexts.

We believe that our success in exposing misconceptions about autistic teenagers' online privacy and safety is largely attributable to our inclusive research design. Unlike previous research that collected information about autistic users' online activities through third-parties (e.g., caregivers), we directly consulted these users - along with non-autistic teenagers, for comparison - to gain a realistic understanding of their perceptions of and experiences with online risks, and identify any unique barriers they may face with protecting their personal information on SNS. Our decision to conduct semi-structured interviews with this population, instead of administering surveys, provided us the flexibility to rephrase our questions when they were unclear and encourage participants to elaborate on their responses while also ensuring their comfort to disclose. Our use of SNS scenario videos as prompts for engaging participants in conversation appeared to be useful in mitigating ambiguity in our questions, thereby enhancing the quality of our data. Our findings may inform the development 
of user-centered approaches to making SNS safer and more inclusive for autistic users.

\subsubsection{Disparities in the Benefits and Risks of Online Social Networking}

Previous research suggests that the benefits and risks of SNS use are inequitably distributed between autistic and non-autistic teenagers. Conceptually, autistic teenagers can benefit equally - if not more - from anonymous computer-mediated communication than the general population to increase their social capital and psychological well-being $[12,20,89]$. However, research shows that most autistic people use SNS less frequently and actively than their typically developing peers [68,70], suggesting that the majority of these users are not taking advantage of these platforms as much as the general population. At the same time, studies demonstrate that autistic teenagers are more at risk of cyberbullying than those without special needs $[20,50,88,130]$. This risk is especially prevalent among the minority of autistic teenagers who use SNS excessively to cope with their loneliness and co-morbid mental illnesses $[4,12]$. Unfortunately, we observed similar disparities in the positive and negative outcomes of online social networking in our research.

\section{Disparities in Benefits}

As anticipated, most of the autistic teenagers in our study did not benefit as much from SNS as their typically developing counterparts. In line with previous research $[68,70]$, we found that non-autistic teenagers prioritized social media use much more than autistic users. Whereas nearly all the non-autistic teenagers used SNS multiple times per day, half of autistic teenagers only logged in occasionally (i.e., a few times a week or less frequently). Autistic teenagers also used SNS more passively than their typically developing counterparts. Most non-autistic teenagers made an effort to increase their social capital by building an authentic profile and engaging with others on SNS. Conversely, the majority of autistic teenagers rarely posted personal content or had deep conversations with people they do not know online. In turn, fewer autistic teenagers had strong online friendships than their typically developing counterparts. These findings are consistent with the "rich-gets-richer, poor-gets-poorer"

phenomenon on social media $[25,92]$, whereby users who have a solid social network 
offline ("rich") tend to benefit more from SNS than those who have low social wellbeing in real life ("poor"). Unfortunately, autistic teenagers are more likely to fall into the latter group than the general population.

\section{Disparities in Risks}

Considering that autistic teenagers use SNS less frequently and actively than their non-autistic peers, it logically follows that autistic users are safer from social privacy threats on these platforms than the general population. For the majority of autistic teenagers who do not interact with people they do not know or trust on SNS, this is in fact the case. However, those who are open and active on social media - most likely to reduce their loneliness - are at exceptional risk of online harassment. Indeed, consistent with previous research $[20,50,88]$, we found that a greater proportion of autistic teenagers reported being cyberbullied — particularly by strangers — than non-autistic teenagers. Moreover, autistic teenagers were much more concerned about being cyberbullied by people they know in real life than their typically developing counterparts. In many cases, these experiences and fears deterred autistic teenagers from pursuing online connections and expressing themselves on SNS, further hindering these users from fully benefiting from these platforms.

\section{Explaining the Disparities}

Based on our research, there are several factors contributing the aforementioned disparities in the outcomes of SNS use for autistic and non-autistic teenagers. First and foremost is autistic teenagers' unique personal characteristics, particularly as they relate to their social skills. Considering that autism is primarily characterized by challenges with navigating social situations [5], it is unsurprising that autistic teenagers struggle to seamlessly integrate themselves into interpersonal interactions both online and offline. Although some autistic teenagers may actually want to connect and build strong relationships on SNS, many do not feel comfortable doing so due to fear of negative evaluation or rejection. Those who have the motivation and confidence to openly express their opinions and feelings online often end up inadvertently causing controversy due to their limited social skills (e.g., predicting others' 
reactions to posts, extreme honesty) and their difficulties with emotion regulation. In turn, most autistic teenagers are at an extreme disadvantage in terms of developing a satisfying social life online compared to non-autistic users.

Autistic teenagers' social engagement online is further impaired by design elements of SNS. This barrier not only relates to complex language conventions of mainstream communication platforms — as discussed in previous research $[43,96,103]$ — but also the social values and norms encouraged by the sites. SNS cater to non-autistic users' social values, such as increasing social capital, enhancing social status, as well as receiving attention and approval. Users' success in living up to these standards is visibly represented through metrics (e.g., number of "likes", followers) embedded within the sites' design that make it easy to compare users' likeability, credibility and influence. Naturally, most non-autistic teenagers readily adhere to these values and develop complex social protocols/norms (e.g., follow-for-follow) to avoid being perceived as a social outcast. Autistic teenagers, on the other hand, do not want to participate in this popularity contest: they just want to stay in touch with their friends, showcase their talents and experiences, and maybe connect with others who have similar interests. However, as shown in our research, autistic teenagers are not entirely unaffected by the social pressures of the virtual world. This pressure may partially explain why autistic teenagers enjoy using SNS less than the general population, as demonstrated in our study and in previous research [7,38].

Furthermore, autistic teenagers' engagement in online social activities — and, in turn, the positive and negative outcomes of their SNS use - are heavily affected by their parents' support and influence. Although our study did not examine parents' perspectives and experiences, previous research shows that most caregivers are exceptionally concerned about their autistic child's safety online and usually rely on restrictive mediation practices to shield their son or daughter from harm [48]. These parents' privacy concerns and protective strategies likely impact their autistic teenagers' online social participation in two main ways. Firstly, these parents may express their extreme privacy and safety concerns to their autistic child, who may then interpret their warnings very literally $[71,113]$ and establish inflexible risk-mitigation 
rules guiding their online social behaviours [62]. Secondly, parents' restrictive mediation practices may stunt autistic teenagers' development of strategies to prevent and deal with threats on SNS independently. This point echoes Wasserman's [127] argument that people with intellectual disabilities — including those with autism can learn valuable lessons from overcoming online privacy risks; therefore, these users should be provided opportunities to engage in positive risk-taking on SNS.

It is important to keep in mind that the autistic participants in our study only represent the members of this population who are actually permitted to use SNS. Indeed, during recruitment, we were approached by a couple of parents who admitted that they did not allow their autistic teenager to use the SNS involved in our study due to safety concerns. It is also not a coincidence that most of our autistic participants were older teenagers; this observation is consistent with previous research indicating that autistic people tend to start using the Internet for social activities at an older age than the general population [68], probably due to restrictive parental mediation. Taken together, efforts must be made to decrease the disparities in online social participation within the autistic population, as well as address the aforementioned differences in online social networking outcomes between autistic and non-autistic youth.

\subsubsection{Balancing Online Privacy and Sociability}

Thus far in our discussion, we have painted a rather bleak picture of the outcomes of autistic teenagers' SNS use. Ultimately, our findings highlight an urgent need for solutions to enhance autistic users' social participation and inclusion online. Some readers may argue that such efforts are futile, given that autistic users are minorities with apparently low interest in engaging in online social activities. We respectfully disagree with this point-of-view. Although most of the autistic teenagers were relatively asocial on SNS, they clearly desired to be included in online society; otherwise, they would not have been motivated to sign up and learn how to use the sites. There is also an ethical imperative to make SNS more inclusive for this population. Indeed, O'Brolcháin and Gordijn [77] argue that technologies should be made available and accessible to people with intellectual disabilities on the basis of distributive justice. 
Even though autistic people only account for less than 2 percent of the world's population, these individuals' needs and desires should still be addressed in the development of technologies, including SNS. Furthermore, efforts to reduce cyberbullying and social anxiety online - issues that are particularly relevant for the autistic population - are invaluable from a mental health perspective.

Inevitably, any initiative to increase autistic teenagers' social participation and inclusion on the Internet must also account for the most fundamental challenge with SNS - that is, balancing privacy and sociability. As demonstrated by our research, both the risks and potential benefits of SNS use are at higher stakes for autistic teenagers than the general population.

\subsubsection{Inter-relatedness of Accessibility, Privacy and Autonomy}

Another key finding in our research relates to the inter-relatedness of cognitive accessibility, online privacy, and autonomy. Cognitive accessibility has been defined as "the extent to which products, systems, services, environments and facilities can be used by people from a population with the widest range of cognitive characteristics and capabilities to achieve a specified goal in a specified context of use" [46]. Based on our findings, it is clear that mainstream SNS have severe cognitive accessibility issues with their designs. Indeed, the autistic participants in our sample had greater difficulty locating and understanding features on SNS than the non-autistic teenagers, suggesting that these platforms are not designed to accommodate neurodiversity. These cognitive accessibility issues not only limit autistic users' ability to use and therefore benefit from the sites, but also prevent them from adjusting their privacy settings independently and in their preferred way. As demonstrated by our research, autistic teenagers require more support to use the privacy settings on SNS than their typically developing counterparts, most likely because these tools are not designed with autistic users' unique cognitive profile in mind.

Moreover, autistic teenagers' autonomy in adjusting their privacy settings is sometimes further subverted by their family members and peers. In line with previous research [48], some of the autistic teenagers in our study reported feeling pressured to make their account private to appease their (over-)protective parents. Moreover, 
several autistic participants described that their family members or peers adjusted their privacy settings for them instead of teaching them how to do it themselves. In some cases, this unintentional affront to autistic teenagers' autonomy caused these users to experience undue distress.

Increased need for autonomy is a core element of adolescent development [134]. Autistic teenagers - much like their typically developing peers — require autonomy to increase their self-efficacy [28]. Unfortunately, the inaccessibility of mainstream SNS is preventing autistic teenagers from having full control over their privacy negotiations with the sites, as mentioned in previous research [89]. These findings highlight the importance of improving the cognitive accessibility of SNS.

\subsubsection{Accessibility is for Everyone}

Cognitive accessibility has yet to become a priority in mainstream SNS companies' design processes. Despite the development of numerous cognitive accessibility design guidelines [37,85,90,105] and related legislation (e.g., Accessible Canada Act), there is still a lack of initiative in the Web development community to invite people with cognitive disabilities — including autistic teenagers — to participate in usability testing. This is unfortunate, not only for people with cognitive disabilities, but for all end-users of SNS. As demonstrated by our research, even non-autistic teenagers experience some difficulties with using the privacy settings on SNS (e.g., remembering their friends' settings) due to cognitive accessibility issues with the sites. Therefore, it would be in SNS companies' best interest to make their platforms more user-friendly for autistic people because doing so would likely improve everyone's user experience.

\subsection{Recommendations}

Based on our research, it is clear that a substantial amount of work must be done to improve the privacy, safety and inclusion autistic teenagers on SNS. In the following sections, we offer recommendations for addressing the factors affecting these users' privacy attitudes and behaviours on SNS identified in our study. All of these recommendations can improve non-autistic teenagers' experiences on SNS as well. 


\subsubsection{Addressing Personal Factors}

\section{Incorporate sentiment nudges}

The high risks associated with autistic teenagers SNS use is largely due to a mismatch between these users' personal disposition and the online social world. As previously discussed, autistic teenagers struggle to interpret other users' the mental states (e.g., beliefs, intentions, emotions) on these platforms. In turn, these users are prone to inadvertently stir up controversy on SNS and become the targets of cyberbullying. One solution to this issue is Wang et al.'s [125] sentiment nudge. A sentiment nudge is a privacy tool that predicts the valence of other people's emotional reactions to users' posts, and provides feedback (e.g., "other people may perceive your post as negative") to dissuade users from sharing controversial content. Conceptually, this technology may help to compensate for the social cognitive limitations (e.g., theory of mind) that cause autistic teenagers to become involved in conflict on SNS. The effectiveness and acceptability of sentiment nudges for protecting autistic social media users from cyberbullying and other social privacy threats has yet to be investigated; however, this could be a promising area for future research.

\section{Provide social skills training}

Another potential method of reducing autistic teenagers' risk of cyberbullying is to offer these users social skills training that is specifically targeted towards encouraging prosocial behaviours in online social situations. As demonstrated by previous research [53], SNS provide autistic teenagers a comfortable environment to practice interacting with others. Future studies should examine the potential to augment this experiential social skills learning through software providing non-intrusive, real-time feedback and advice to autistic social media users. This technology should be developed in collaboration with autistic teenagers to ensure the relevance and accessibility of the tool's content, as well as prevent any unintentional negative outcomes (e.g., loss of autonomy, stress, annoyance) of its use. 


\section{Include trustworthiness metrics}

Much like the general population, autistic teenagers rely on their intuition to determine who they can trust on SNS. Partially due to their high privacy concerns and general aversion to risks, most autistic teenagers choose to avoid interacting with strangers online altogether, thereby preventing them from building relationships online. A potential solution to this problem is the addition of user trustworthiness metrics on social media profiles, similar to those on ebay and Wikipedia [20]. Burke et al. [20] envision this feature to display attributes of potential online friends (e.g., if they have a legitimate email address, socialize with same people for long periods of times) that could aid in autistic users' decision-making regarding who to avoid or approach. This solution may be useful in relieving autistic teenagers' privacy concerns, thereby increasing their willingness to engage in social interactions. At the same time, trustworthiness metrics may backfire by providing inaccurate assessments if online predators manage to trick the indicators. Furthermore, these tools may unintentionally damage autistic teenagers' online reputation and, in turn, their likelihood of building positive relationships on SNS if they are carelessly designed. Autistic teenagers are more likely to unintentionally perpetrate cyberbullying on social media than the general population [50]. Therefore, if trustworthiness metrics account for engagement in cyberbullying and other social indicators (e.g., active use of SNS), many autistic teenagers would have a low trustworthiness score and may therefore be discriminated against in online social situations. Again, if researchers were to pursue this solution, they must do so with autistic teenagers' personal disposition in mind.

\section{Decrease the visibility of social evaluation elements on the interface}

Our research highlights the need to redesign SNS to be more accommodating towards autistic users' social protocols and values. Specifically, SNS designers should consider reducing the amount of publicly visible social evaluation elements (e.g., number of "likes" and followers) on the interface. This would be a welcomed change for both autistic and non-autistic teenagers who are high in social anxiety. 


\subsubsection{Addressing Social Factors}

\section{Develop collaborative tools to facilitate parent-teenager communication about privacy protection on social networking sites}

Family members, peers, and teachers all play an important role in supporting autistic teenagers' privacy protection on SNS. As shown in our research, autistic teenagers require more support to protect their privacy on SNS than the general population; yet, they should have the same right to autonomy as their typically developing peers. Researchers have proposed solutions that balance autistic users' need for support and autonomy. For instance, Just and Berg [48] advocate for the development of collaborative tools that can facilitate communication between autistic teenagers and their parents regarding privacy protection on SNS. Similarly, some of the adults with intellectual disabilities in Shpigelman and Gill's [98] study suggested "adding a function of online help guided by a trusted advisor or mentor" to SNS.

\section{Facilitate online privacy and safety support groups}

Several researchers endorse the development and implementation of support groups devoted to teaching people with intellectual disabilities how to deal with online risks $[22,43]$. For example, Holmes and O'Loughlin [43] describe an offline group that offers support and advice to Internet users with intellectual disabilities related to protecting their privacy and safety online. Using a psycho-educational approach, the support group provides these users a safe and non-judgmental space to talk about their negative online experiences. These open discussions are followed-up with informal courses teaching people with intellectual disabilities practical privacy protection skills (e.g., how to report abuse and manage privacy settings). To improve the availability of these support groups, online versions (e.g., on Faceboook, forums) may be formed; however, these groups would need to be strictly moderated to protect participants from malicious actors who may see these groups as easy targets. 


\section{Develop online privacy-related interactive e-books}

Researchers have examined the potential for picture-rich educational tools, such as interactive e-books, in teaching non-autistic children about online privacy with promising results [132]. Parallel age-appropriate educational resources may reduce the pressure on parents and teachers to educate their autistic teenagers about online privacy, especially if these resources include text-to-speech functions.

\section{Promote active parental mediation}

Currently, there is a need for research-informed resources to help parents to protect their autistic teenagers' privacy, while also promoting positive risk-taking. A future output of this study will be an information sheet providing this advice, based on the insights from our study that we previously discussed.

\section{Encourage the Web community to prioritize cognitive accessibility}

Most importantly, efforts must be made to reduce autistic teenagers' need for support to use SNS by increasing the cognitive accessibility of these platforms. SNS designers have tremendous control over empowering or disabling autistic users of their sites. Improving the cognitive accessibility of SNS would require considerable reforms and cooperation in the Web community at large. To increase the Web community's commitment to making SNS accessible to autistic people, there is a need to demonstrate the benefits of this initiative for all stakeholders through further scientific research, and provide actionable advice for shifting their practices.

\subsubsection{Addressing Contextual Factors}

\section{Provide flexible privacy settings}

As shown in our research, most users' privacy concerns and protective behaviours vary depending on the disclosure context - specifically, the audience and type of content intended to be disclosed. On most SNS, users only have the option to either make their content entirely public or private. This limited granularity often causes users to engage in coping strategies, such as creating separate accounts to manage 
their public and private personas. These findings demonstrate a need to offer more flexible privacy settings on SNS; this may not only enhance users' privacy, but may also increase their self-disclosure, as discussed in previous research $[111,131]$.

\subsubsection{Addressing Social Networking Site Design Factors}

\section{Improve the cognitive accessibility of mainstream social networking sites}

Clearly, mainstream SNS are not usable for autistic teenagers. Some researchers believe that the solution to this problem is to develop specialized SNS for people with cognitive disabilities. An example is Tuset et al.'s [115] instant messaging service called Messenger Visual, which uses pictograms (i.e., pictures conveying a meaning) as the main language instead of traditional letter-based words to enable communication for people with low literacy. Although specialized SNS, like Messenger Visual, can be very useful for some, their development should not replace efforts to make mainstream social media more accommodating to neurodiversity. Specialized assistive technologies may be stigmatizing for users with cognitive disabilities [86], who have the desire and right to benefit from equivalent services to those used by the general population. Indeed, a psychological benefit of social media use by users with intellectual disabilities - including those with autism - is the sense of pride and inclusion that comes along with being able to use SNS like Facebook just like everybody else [96]. Therefore, the focus of future research and initiatives should be to make SNS as accessible and inclusive as possible for users with a wide range of cognitive capabilities.

\subsection{Limitations and Future Work}

Inevitably, this study has limitations. Firstly, our participants consisted of a very small sample of autistic and non-autistic teenagers, due to challenges with recruitment. Therefore, the generalizability of our findings is uncertain. That said, we

believe that we reached saturation with both groups' responses. Future research could replicate our work with more participants. Secondly, our semi-structured interviews were guided by only a few scenarios representing a limited set of social privacy 
risks on SNS. Future studies could extend the scope of our research by examining autistic and non-teenagers' privacy attitudes and behaviours in response to a wider range of online privacy threats. Furthermore, to improve the ecological validity of our findings, researchers could collect objective and observational data of autistic and non-autistic teenagers' online activities. Finally, it is important to acknowledge that SNS use should not replace face-to-face interactions, but rather serve as a supplement to offline relationships. Future research in this area should consider the influence of improving the design of SNS for autistic users on their offline social functioning.

\subsection{Conclusion}

Previous studies highlight excessive privacy and safety risks associated with autistic teenagers' SNS use $[39,48]$; however, researchers had yet to examine why this may be the case. The goal of our study was to address this research gap and provide recommendations for making autistic teenagers safer on SNS.

Through our semi-structured interviews with autistic and non-autistic teenagers, we gained insight into our autistic participants' thoughts and feelings about their privacy on SNS, and their strategies for protecting their personal information and safety online $(R Q 1)$. We also identified key factors affecting these users' privacy attitudes and behaviors on SNS (RQ2). Finally, we successfully compared our autistic teenagers' privacy attitudes and behaviours, as well as the factors affecting their privacy, on SNS with those reported by non-autistic teenagers (RQ3).

In response to our research questions, we found that the autistic teenagers in our sample were highly concerned about their privacy on SNS and employed many strategies to mitigate risks $(R Q 1)$. Their privacy attitudes and behaviours were affected by several personal, social, contextual, and SNS design factors (RQ2). Autistic teenagers' experiences with SNS and their perceptions of online privacy were noticeably distinct from those reported by their typically developing counterparts. Whereas most autistic teenagers prioritized their privacy over self-disclosure on SNS, the majority of non-autistic teenagers attempted to balance the risks and benefits of online social networking. Although both groups were affected by similar privacy-related factors, autistic teenagers' personal disposition and increased need for support to use 
SNS - due to cognitive accessibility issues with the platforms — prevented them from benefiting from these sites as much as the general population (RQ3).

At the beginning of this study, we fully expected our findings to highlight a need to address severe privacy and safety concerns related to autistic teenagers' SNS use. However, we eventually came to realize that we may have been focusing on the wrong issue all along. Contrary to popular belief, the autistic teenagers in our study did not appear to be more vulnerable to most social privacy threats on SNS (except cyberbullying) than their typically developing peers. However, they were more susceptible to a different threat to their social and emotional well-being than typically developing youth - that is, social exclusion. The real world is not the most inviting place for autistic teenagers, but the virtual world does not have to be that way too. Through inclusive research and collaborations with user experience designers in industry, we can make SNS both safe and enjoyable for these users with special needs. 


\section{Bibliography}

[1] Denise E Agosto and June Abbas. "Don't be dumb - that's the rule I try to live by": A closer look at older teens' online privacy and safety attitudes. New Media \& Society, 19(3):347-365, 2017.

[2] Kelly A Allen, Tracii Ryan, DeLeon L Gray, Dennis M McInerney, and Lea Waters. Social media use and social connectedness in adolescents: The positives and the potential pitfalls. The Educational and Developmental Psychologist, 31(1):18-31, 2014.

[3] Monica Anderson and Jingjing Jiang. Teens, social media \& technology 2018. Pew Research Center, 31, 2018.

[4] Rebecca P Ang. Adolescent cyberbullying: A review of characteristics, prevention and intervention strategies. Aggression and violent behavior, 25:35-42, 2015 .

[5] American Psychiatric Association et al. Diagnostic and statistical manual of mental disorders (DSM-5®). American Psychiatric Pub, 2013.

[6] Elizabeth J Austin. Personality correlates of the broader autism phenotype as assessed by the Autism Spectrum Quotient (AQ). Personality and Individual Differences, 38(2):451-460, 2005.

[7] Khadija Bahiss, Sally Jo Cunningham, and Tony Smith. Investigating the usability of social networking sites for teenagers with autism. In Proceedings of the 11th International Conference of the NZ Chapter of the ACM Special Interest Group on Human-Computer Interaction, pages 5-8. ACM, 2010.

[8] Jon Baio, Lisa Wiggins, Deborah L Christensen, Matthew J Maenner, Julie Daniels, Zachary Warren, Margaret Kurzius-Spencer, Walter Zahorodny, Cordelia Robinson Rosenberg, Tiffany White, et al. Prevalence of autism spectrum disorder among children aged 8 years - Autism and Developmental Disabilities Monitoring Network, 11 Sites, United States, 2014. MMWR Surveillance Summaries, 67(6):1, 2018.

[9] Claire Balleys and Sami Coll. Being publicly intimate: Teenagers managing online privacy. Media, Culture \&6 Society, 39(6):885-901, 2017.

[10] Valerie Barker. Older adolescents' motivations for social network site use: The influence of gender, group identity, and collective self-esteem. Cyberpsychology $\&$ behavior, 12(2):209-213, 2009. 
[11] Simon Baron-Cohen. Theory of mind and autism: A review. In International review of research in mental retardation, volume 23, pages 169-184. Elsevier, 2000 .

[12] Penny Benford and P Standen. The Internet: A comfortable communication medium for people with Asperger syndrome (AS) and high functioning autism (HFA)? Journal of Assistive Technologies, 3(2):44-53, 2009.

[13] Ine Beyens, Eline Frison, and Steven Eggermont. "I don't want to miss a thing": Adolescents' fear of missing out and its relationship to adolescents' social needs, facebook use, and facebook related stress. Computers in Human Behavior, 64:1-8, 2016.

[14] David Blackwell, Carrie Leaman, Rose Tramposch, Ciera Osborne, and Miriam Liss. Extraversion, neuroticism, attachment style and fear of missing out as predictors of social media use and addiction. Personality and Individual Differences, 116:69-72, 2017.

[15] Danah M Boyd and Nicole B Ellison. Social network sites: Definition, history, and scholarship. Journal of computer-mediated Communication, 13(1):210-230, 2007.

[16] Petter Bae Brandtzæg, Marika Lüders, and Jan Håvard Skjetne. Too many Facebook "friends"? Content sharing and sociability versus the need for privacy in social network sites. Intl. Journal of Human-Computer Interaction, 26(1112):1006-1030, 2010.

[17] Virginia Braun and Victoria Clarke. Using thematic analysis in psychology. Qualitative research in psychology, 3(2):77-101, 2006.

[18] Jo Bryce and James Fraser. The role of disclosure of personal information in the evaluation of risk and trust in young peoples' online interactions. Computers in Human Behavior, 30:299-306, 2014.

[19] Petra CM Buijs, Erik Boot, Andrea Shugar, Wai Lun Alan Fung, and Anne S Bassett. Internet safety issues for adolescents and adults with intellectual disabilities. Journal of Applied Research in Intellectual Disabilities, 30(2):416-418, 2017.

[20] Moira Burke, Robert Kraut, and Diane Williams. Social use of computermediated communication by adults on the autism spectrum. In Proceedings of the 2010 ACM conference on Computer supported cooperative work, pages 425-434. ACM, 2010.

[21] Sue Caton and Melanie Chapman. The use of social media and people with intellectual disability: A systematic review and thematic analysis. Journal of Intellectual and Developmental Disability, 41(2):125-139, 2016. 
[22] Darren D Chadwick, Sally Quinn, and Chris Fullwood. Perceptions of the risks and benefits of Internet access and use by people with intellectual disabilities. British Journal of Learning Disabilities, 45(1):21-31, 2017.

[23] Sangmi Chai, Sharmistha Bagchi-Sen, Claudia Morrell, H Raghav Rao, and Shambhu J Upadhyaya. Internet and online information privacy: An exploratory study of preteens and early teens. IEEE Transactions on Professional Communication, 52(2):167-182, 2009.

[24] Hajer Chalghoumi, Virginie Cobigo, and Jeffrey Jutai. Ethical issues related to IT adoption by elderly persons with cognitive impairments. In AAATE Conf., pages 59-63, 2017.

[25] Gina Masullo Chen. Revisiting the social enhancement hypothesis: Extroversion indirectly predicts number of Facebook friends operating through Facebook usage. Computers in Human Behavior, 39:263-269, 2014.

[26] Hongliang Chen, Christopher E Beaudoin, and Traci Hong. Teen online information disclosure: Empirical testing of a protection motivation and social capital model. Journal of the association for information science and technology, 67(12):2871-2881, 2016.

[27] Emily Christofides, Amy Muise, and Serge Desmarais. Risky disclosures on Facebook: The effect of having a bad experience on online behavior. Journal of adolescent research, 27(6):714-731, 2012.

[28] Elaine Clark, Daniel E Olympia, Jenise Jensen, Lora Tuesday Heathfield, and William R Jenson. Striving for autonomy in a contingency-governed world: Another challenge for individuals with developmental disabilities. Psychology in the Schools, 41(1):143-153, 2004.

[29] Teresa Correa, Amber Willard Hinsley, and Homero Gil De Zuniga. Who interacts on the Web?: The intersection of users' personality and social media use. Computers in human behavior, 26(2):247-253, 2010.

[30] Katie Davis. Friendship 2.0: Adolescents' experiences of belonging and selfdisclosure online. Journal of adolescence, 35(6):1527-1536, 2012.

[31] Anne Deckers, Peter Muris, and Jeffrey Roelofs. Being on your own or feeling lonely? Loneliness and other social variables in youths with autism spectrum disorders. Child Psychiatry \&6 Human Development, 48(5):828-839, 2017.

[32] Amandeep Dhir, Puneet Kaur, Kirsti Lonka, and Marko Nieminen. Why do adolescents untag photos on Facebook? Computers in Human Behavior, 55:1106$1115,2016$. 
[33] Guadalupe Espinoza and Jaana Juvonen. The pervasiveness, connectedness, and intrusiveness of social network site use among young adolescents. $C y$ berpsychology, Behavior, and Social Networking, 14(12):705-709, 2011.

[34] E Fambonne. Epidemiology of autistic disorder and other pervasive developmental disorders. J Clin Psychiat, 66:3-8, 2005.

[35] Marisa H Fisher, Andrew L Moskowitz, and Robert M Hodapp. Differences in social vulnerability among individuals with autism spectrum disorder, Williams syndrome, and Down syndrome. Research in Autism Spectrum Disorders, 7(8):931-937, 2013.

[36] Sue Fletcher-Watson and Francesca Happé. Autism: A new introduction to psychological theory and current debate. Routledge, 2019.

[37] Mark G Friedman and Diane Nelson Bryen. Web accessibility design recommendations for people with cognitive disabilities. Technology and Disability, 19(4):205-212, 2007.

[38] Kristen Gillespie-Lynch, Steven K Kapp, Christina Shane-Simpson, David Shane Smith, and Ted Hutman. Intersections between the autism spectrum and the Internet: Perceived benefits and preferred functions of computer-mediated communication. Intellectual and developmental disabilities, 52(6):456-469, 2014.

[39] Bethany Good and Lin Fang. Promoting smart and safe Internet use among children with neurodevelopmental disorders and their parents. Clinical Social Work Journal, 43(2):179-188, 2015.

[40] Wannes Heirman, Michel Walrave, Anne Vermeulen, Koen Ponnet, Heidi Vandebosch, and Kris Hardies. Applying the Theory of Planned Behavior to adolescents' acceptance of online friendship requests sent by strangers. Telematics and Informatics, 33(4):1119-1129, 2016.

[41] Albert A Herzog Jr. Spires, wheelchairs and committees: Organizing for disability advocacy at the judicatory level. Review of religious research, pages 349-367, 2004.

[42] Bas Hofstra, Rense Corten, and Frank van Tubergen. Understanding the privacy behavior of adolescents on Facebook: The role of peers, popularity and trust. Computers in Human Behavior, 60:611-621, 2016.

[43] Katrina M Holmes and Nessa O'loughlin. The experiences of people with learning disabilities on social networking sites. British Journal of Learning Disabilities, 42(1):1-5, 2014. 
[44] Pier Jaarsma and Stellan Welin. Autism as a natural human variation: Reflections on the claims of the neurodiversity movement. Health Care Analysis, 20(1):20-30, 2012.

[45] A Jawaid, DM Riby, J Owens, SW White, T Tarar, and PE Schulz. "too withdrawn" or "too friendly": Considering social vulnerability in two neurodevelopmental disorders. Journal of Intellectual Disability Research, 56(4):335$350,2012$.

[46] Stefan Johansson. Towards a framework to understand mental and cognitive accessibility in a digital context. PhD thesis, KTH Royal Institute of Technology, 2016 .

[47] Matthew Johnson. To Share or Not to Share: How Teens Make Privacy Decisions about Photos on Social Media. Media Smarts, 2017.

[48] Mike Just and Tessa Berg. Keeping children safe online: Understanding the concerns of carers of children with autism. In IFIP Conference on HumanComputer Interaction, pages 34-53. Springer, 2017.

[49] Rachel Kelly, Mary-Pat OMalley, and Stanislava Antonijevic. "Just trying to talk to people... its the hardest": Perspectives of adolescents with highfunctioning autism spectrum disorder on their social communication skills. Child Language Teaching and Therapy, 34(3):319-334, 2018.

[50] Robin M Kowalski and Cristin Fedina. Cyber bullying in ADHD and Asperger syndrome populations. Research in Autism Spectrum Disorders, 5(3):12011208, 2011.

[51] Robin M Kowalski and Susan P Limber. Electronic bullying among middle school students. Journal of adolescent health, 41(6):S22-S30, 2007.

[52] Taylor E Krcek. Deconstructing disability and neurodiversity: Controversial issues for autism and implications for social work. Journal of Progressive Human Services, 24(1):4-22, 2013.

[53] Jay Kuder and Joy Xin. Using Facebook to improve social communication skills of students with autism. In EdMedia + Innovate Learning, pages 10381044. Association for the Advancement of Computing in Education (AACE), 2013.

[54] Melissa H Kuo, Gael I Orsmond, Wendy J Coster, and Ellen S Cohn. Media use among adolescents with autism spectrum disorder. Autism, 18(8):914-923, 2014. 
[55] Sanna Kuusikko, Rachel Pollock-Wurman, Katja Jussila, Alice S Carter, MarjaLeena Mattila, Hanna Ebeling, David L Pauls, and Irma Moilanen. Social anxiety in high-functioning children and adolescents with autism and Asperger syndrome. Journal of autism and developmental disorders, 38(9):1697-1709, 2008.

[56] Frederik Kydland, Judith Molka-Danielsen, and Susan Balandin. Examining the use of social media tool "Flickr" for impact on loneliness for people with intellectual disability. In NOKOBIT 2012, pages 253-264, 2012.

[57] G La Malfa, S Lassi, M Bertelli, R Salvini, and GF Placidi. Autism and intellectual disability: A study of prevalence on a sample of the Italian population. Journal of intellectual disability research, 48(3):262-267, 2004.

[58] Linda A LeBlanc, Andrew R Riley, and Tina R Goldsmith. Autism spectrum disorders: A lifespan perspective. In Clinical assessment and intervention for autism spectrum disorders, pages 65-87. Elsevier, 2008.

[59] Bianca W Lee and Lexine A Stapinski. Seeking safety on the internet: Relationship between social anxiety and problematic Internet use. Journal of anxiety disorders, 26(1):197-205, 2012.

[60] Louis Leung. Predicting Internet risks: A longitudinal panel study of gratifications-sought, Internet addiction symptoms, and social media use among children and adolescents. Health Psychology and Behavioral Medicine: An Open Access Journal, 2(1):424-439, 2014.

[61] Louis Leung and Paul SN Lee. The influences of information literacy, Internet addiction and parenting styles on Internet risks. New Media \& Society, 14(1):117-136, 2012.

[62] Irwin P Levin, Gary J Gaeth, Megan Foley-Nicpon, Vitaliya Yegorova, Charles Cederberg, and Haoyang Yan. Extending decision making competence to special populations: A pilot study of persons on the autism spectrum. Frontiers in psychology, 6:539, 2015.

[63] Cong Liu, Rebecca P Ang, and May O Lwin. Cognitive, personality, and social factors associated with adolescents' online personal information disclosure. Journal of adolescence, 36(4):629-638, 2013.

[64] Sonia Livingstone. Taking risky opportunities in youthful content creation: Teenagers' use of social networking sites for intimacy, privacy and selfexpression. New media \& society, 10(3):393-411, 2008.

[65] Jill Locke, Eric H Ishijima, Connie Kasari, and Nancy London. Loneliness, friendship quality and the social networks of adolescents with high-functioning 
autism in an inclusive school setting. Journal of Research in Special Educational Needs, 10(2):74-81, 2010.

[66] Lotta Löfgren-Mårtenson, Emma Sorbring, and Martin Molin. T@ngled up in blue: Views of parents and professionals on internet use for sexual purposes among young people with intellectual disabilities. Sexuality and disability, $33(4): 533-544,2015$.

[67] May O Lwin, Benjamin Li, and Rebecca P Ang. Stop bugging me: An examination of adolescents' protection behavior against online harassment. Journal of adolescence, 35(1):31-41, 2012.

[68] Jennifer A MacMullin, Yona Lunsky, and Jonathan A Weiss. Plugged in: Electronics use in youth and young adults with autism spectrum disorder. Autism, 20(1):45-54, 2016.

[69] Micah O Mazurek. Social media use among adults with autism spectrum disorders. Computers in Human Behavior, 29(4):1709-1714, 2013.

[70] Micah O Mazurek, Paul T Shattuck, Mary Wagner, and Benjamin P Cooper. Prevalence and correlates of screen-based media use among youths with autism spectrum disorders. Journal of autism and developmental disorders, 42(8):17571767, 2012.

[71] Peter Mitchell, Rebecca Saltmarsh, and Helen Russell. Overly literal interpretations of speech in autism: Understanding that messages arise from minds. Journal of Child Psychology and Psychiatry, 38(6):685-691, 1997.

[72] Martin Molin, Emma Sorbring, and Lotta Löfgren-Mårtenson. Teachers' and parents' views on the Internet and social media usage by pupils with intellectual disabilities. Journal of Intellectual Disabilities, 19(1):22-33, 2015.

[73] Ajaya Neupane, Kiavash Satvat, Nitesh Saxena, Despina Stavrinos, and Haley Johnson Bishop. Do social disorders facilitate social engineering?: A case study of autism and phishing attacks. In Proceedings of the 34th Annual Computer Security Applications Conference, pages 467-477. ACM, 2018.

[74] Jakob Nielsen. Usability engineering. Elsevier, 1994.

[75] Claude L Normand and François Sallafranque-St-Louis. Cybervictimization of young people with an intellectual or developmental disability: Risks specific to sexual solicitation. Journal of Applied Research in Intellectual Disabilities, $29(2): 99-110,2016$.

[76] Natascha Notten and Peter Nikken. Boys and girls taking risks online: A gendered perspective on social context and adolescents' risky online behavior. New Media \&f Society, 18(6):966-988, 2016. 
[77] Fiachra O'Brolcháin and Bert Gordijn. Persons with intellectual and developmental disabilities and information technologies. Some ethical observations A comment on Chalgoumi et al. Ethics \& Behavior, 29(3):218-222, 2019.

[78] Babajide Osatuyi. Personality traits and information privacy concern on social media platforms. Journal of Computer Information Systems, 55(4):11-19, 2015.

[79] Luci Pangrazio and Neil Selwyn. "It's not like it's life or death or whatever": Young peoples understandings of social media data. Social Media+ Society, 4(3):2056305118787808, 2018.

[80] Frank W Paulus, Charlotte S Sander, Monika Nitze, Anne-Rose KramatschekPfahler, Anette Voran, and Alexander von Gontard. Gaming disorder and computer-mediated communication in children and adolescents with autism spectrum disorder. Zeitschrift für Kinder-und Jugendpsychiatrie und Psychotherapie, 2019.

[81] Jochen Peter, Patti M Valkenburg, and Alexander P Schouten. Developing a model of adolescent friendship formation on the internet. CyberPsychology $\mathcal{B}$ Behavior, 8(5):423-430, 2005.

[82] Neysa Petrina, Mark Carter, and Jennifer Stephenson. The nature of friendship in children with autism spectrum disorders: A systematic review. Research in Autism Spectrum Disorders, 8(2):111-126, 2014.

[83] Kathleen A Quill. Instructional considerations for young children with autism: The rationale for visually cued instruction. Journal of autism and developmental disorders, 27(6):697-714, 1997.

[84] Ana Radovic, Theresa Gmelin, Bradley D Stein, and Elizabeth Miller. Depressed adolescents' positive and negative use of social media. Journal of adolescence, 55:5-15, 2017.

[85] Dora M Raymaker, Steven K Kapp, Katherine E McDonald, Michael Weiner, Elesia Ashkenazy, and Christina Nicolaidis. Development of the AASPIRE Web accessibility guidelines for autistic Web users. Autism in Adulthood, 1(2):146$157,2019$.

[86] Jessica Nicole Rocheleau, Virginie Cobigo, and Hajer Chalghoumi. Recognizing everyday information technologies as assistive technologies for persons with cognitive disabilities. In International Conference on Computers Helping People with Special Needs, pages 504-508. Springer, 2018.

[87] Daniel Romer. Adolescent risk taking, impulsivity, and brain development: Implications for prevention. Developmental Psychobiology: The Journal of the International Society for Developmental Psychobiology, 52(3):263-276, 2010. 
[88] François Sallafranque-St-Louis and Claude L Normand. From solitude to solicitation: How people with intellectual disability or autism spectrum disorder use the Internet. Cyberpsychology: Journal of Psychosocial Research on Cyberspace, 11(1), 2017.

[89] Susan M Schultz, Gloria Jacobs, and Jacob Schultz. A promising practice: using Facebook as a communication and social networking tool. Rural Special Education Quarterly, 32(4):38-44, 2013.

[90] Jane Seale, Helena Garcia-Carrisoza, Jonty Rix, Kieron Sheehy, and Simon Hayhoe. A proposal for a unified framework for the design of technologies for people with learning difficulties. Technology and Disability, 30(1-2):25-40, 2018.

[91] Gwendolyn Seidman. Self-presentation and belonging on Facebook: How personality influences social media use and motivations. Personality and Individual Differences, 54(3):402-407, 2013.

[92] Maarten Selfhout, William Burk, Susan Branje, Jaap Denissen, Marcel Van Aken, and Wim Meeus. Emerging late adolescent friendship networks and Big Five personality traits: A social network approach. Journal of personality, 78(2):509-538, 2010.

[93] Krystelle Shaughnessy, Jessica N Rocheleau, Somayyeh Kamalou, and David A Moscovitch. The effects of social anxiety and online privacy concern on individual differences in Internet-based interaction anxiety and communication preferences. Cyberpsychology, Behavior, and Social Networking, 20(4):212-217, 2017.

[94] Wonsun Shin and Nurzali Ismail. Exploring the role of parents and peers in young adolescents' risk taking on social networking sites. Cyberpsychology, Behavior, and Social Networking, 17(9):578-583, 2014.

[95] Wonsun Shin and May O Lwin. How does "talking about the Internet with others" affect teenagers' experience of online risks? The role of active mediation by parents, peers, and school teachers. New Media $\mathcal{E}$ Society, 19(7):1109-1126, 2017.

[96] Carmit-Noa Shpigelman. Leveraging social capital of individuals with intellectual disabilities through participation on facebook. Journal of Applied Research in Intellectual Disabilities, 31(1):e79-e91, 2018.

[97] Carmit-Noa Shpigelman and Carol J Gill. Facebook use by persons with disabilities. Journal of Computer-Mediated Communication, 19(3):610-624, 2014.

[98] Carmit-Noa Shpigelman and Carol J Gill. How to make online social networks accessible for users with intellectual disability? In International Conference on Computers for Handicapped Persons, pages 471-477. Springer, 2014. 
[99] Cristiana S Silva, Glívia AR Barbosa, Ismael S Silva, Tatiane S Silva, Fernando Mourão, and Flávio Coutinho. Privacy for children and teenagers on social networks from a usability perspective: A case study on Facebook. In Proceedings of the 2017 ACM on Web Science Conference, pages 63-71. ACM, 2017.

[100] John Snakenborg, Richard Van Acker, and Robert A Gable. Cyberbullying: Prevention and intervention to protect our children and youth. Preventing School Failure: Alternative Education for Children and Youth, 55(2):88-95, 2011.

[101] Tal Soffer and Anat Cohen. Privacy perception of adolescents in a digital world. Bulletin of Science, Technology \& Society, 34(5-6):145-158, 2014.

[102] Kate Sofronoff, Elizabeth Dark, and Valerie Stone. Social vulnerability and bullying in children with Asperger syndrome. Autism, 15(3):355-372, 2011.

[103] Emma Sorbring, Martin Molin, and Lotta Löfgren-Mårtenson. "I'm a mother, but I'm also a facilitator in her every-day life": Parents voices about barriers and support for Internet participation among young people with intellectual disabilities. Cyberpsychology: Journal of Psychosocial Research on Cyberspace; $1,11,2017$.

[104] Marcantonio M Spada. An overview of problematic internet use. Addictive behaviors, 39(1):3-6, 2014.

[105] Emily J Steel and Gunnel Janeslätt. Drafting standards on cognitive accessibility: a global collaboration. Disability and Rehabilitation: Assistive Technology, 12(4):385-389, 2017.

[106] Wouter MP Steijn. A developmental perspective regarding the behaviour of adolescents, young adults, and adults on social network sites. Cyberpsychology: Journal of Psychosocial Research on Cyberspace, 8(2), 2014.

[107] Wouter MP Steijn and Anton Vedder. Privacy under construction: A developmental perspective on privacy perception. Science, Technology, \& Human Values, 40(4):615-637, 2015.

[108] Laurence Steinberg. Risk taking in adolescence: New perspectives from brain and behavioral science. Current directions in psychological science, 16(2):55-59, 2007.

[109] Laurence Steinberg. A social neuroscience perspective on adolescent risk-taking. Developmental review, 28(1):78-106, 2008.

[110] Susannah Stern. Regretted online self-presentations: US college students' recollections and reflections. Journal of Children and Media, 9(2):248-265, 2015. 
[111] Frederic D Stutzman, Ralph Gross, and Alessandro Acquisti. Silent listeners: The evolution of privacy and disclosure on Facebook. Journal of privacy and confidentiality, 4(2):2, 2013.

[112] John Swain, Sally French, and Colin Cameron. Controversial issues in a disabling society. McGraw-Hill Education (UK), 2003.

[113] Helen Tager-Flusberg. Language and understanding minds: Connections in autism. Understanding other minds: Perspectives from developmental cognitive neuroscience, 2:124-149, 2000.

[114] Elda Tartari. Benefits and risks of children and adolescents using social media. European Scientific Journal, ESJ, 11(13), 2015.

[115] Pere Tuset, Juan Miguel López, Pere Barberán, Léonard Janer, and Cristina Cervelló-Pastor. Designing Messenger Visual, an instant messaging service for individuals with cognitive disability. In International Workshop on Ambient Assisted Living, pages 57-64. Springer, 2011.

[116] Mitch van Geel, Anouk Goemans, Fatih Toprak, and Paul Vedder. Which personality traits are related to traditional bullying and cyberbullying? a study with the Big Five, Dark Triad and sadism. Personality and Individual Differences, 106:231-235, 2017.

[117] Ellen Van Gool, Joris Van Ouytsel, Koen Ponnet, and Michel Walrave. To share or not to share? Adolescents' self-disclosure about peer relationships on Facebook: An application of the Prototype Willingness Model. Computers in Human Behavior, 44:230-239, 2015.

[118] Ellen Vanderhoven, Tammy Schellens, Martin Valcke, and Annelies Raes. How safe do teenagers behave on Facebook? an observational study. PLoS One, 9(8):e104036, 2014.

[119] J Mitchell Vaterlaus. Parental mediation of adolescent technology use. In Advanced Methodologies and Technologies in Media and Communications, pages 488-498. IGI Global, 2019.

[120] Philippe Verduyn, David Seungjae Lee, Jiyoung Park, Holly Shablack, Ariana Orvell, Joseph Bayer, Oscar Ybarra, John Jonides, and Ethan Kross. Passive facebook usage undermines affective well-being: Experimental and longitudinal evidence. Journal of Experimental Psychology: General, 144(2):480, 2015.

[121] Philippe Verduyn, Oscar Ybarra, Maxime Résibois, John Jonides, and Ethan Kross. Do social network sites enhance or undermine subjective well-being? a critical review. Social Issues and Policy Review, 11(1):274-302, 2017. 
[122] Anne Vermeulen, Heidi Vandebosch, and Wannes Heirman. \# Smiling,\# venting, or both? Adolescents' social sharing of emotions on social media. Computers in Human Behavior, 84:211-219, 2018.

[123] Michel Walrave and Wannes Heirman. Adolescents, online marketing and privacy: Predicting adolescents willingness to disclose personal information for marketing purposes. Children \& Society, 27(6):434-447, 2013.

[124] Mitzi Waltz. Autism = death: The social and medical impact of a catastrophic medical model of autistic spectrum disorders. Popular narrative media, 1(1):1324,2008 .

[125] Yang Wang, Pedro Giovanni Leon, Kevin Scott, Xiaoxuan Chen, Alessandro Acquisti, and Lorrie Faith Cranor. Privacy nudges for social media: An exploratory Facebook study. In Proceedings of the 22nd International Conference on World Wide Web, pages 763-770. ACM, 2013.

[126] Deborah M Ward, Karen E Dill-Shackleford, and Micah O Mazurek. Social media use and happiness in adults with autism spectrum disorder. Cyberpsychology, Behavior, and Social Networking, 21(3):205-209, 2018.

[127] David Wasserman. A case for greater risk tolerance in Internet use by adults with intellectual disabilities: A comment on chalghoumi et al. Ethics \& Behavior, 29(3):223-226, 2019.

[128] Amanda L Williams and Michael J Merten. A review of online social networking profiles by adolescents: Implications for future research and intervention. ADOLESCENCE-SAN DIEGO-, 43(170):253, 2008.

[129] Pamela Wisniewski, Heng Xu, Mary Beth Rosson, Daniel F Perkins, and John M Carroll. Dear diary: Teens reflect on their weekly online risk experiences. In Proceedings of the 2016 CHI Conference on Human Factors in Computing Systems, pages 3919-3930. ACM, 2016.

[130] Michelle F Wright. Cyber victimization and depression among adolescents with intellectual disabilities and developmental disorders: The moderation of perceived social support. Journal of Mental Health Research in Intellectual Disabilities, 10(2):126-143, 2017.

[131] Philip Fei Wu. The privacy paradox in the context of online social networking: A self-identity perspective. Journal of the Association for Information Science and Technology, 70(3):207-217, 2019.

[132] Leah Zhang-Kennedy, Yomna Abdelaziz, and Sonia Chiasson. Cyberheroes: The design and evaluation of an interactive ebook to educate children about online privacy. International Journal of Child-Computer Interaction, 13:10-18, 2017. 
[133] Gila Cohen Zilka. Awareness of eSafety and potential online dangers among children and teenagers. Journal of Information Technology Education: Research, 16:319-338, 2017.

[134] Melanie J Zimmer-Gembeck and W Andrew Collins. Autonomy development during adolescence. Blackwell handbook of adolescence, 8:175, 2008.

[135] Eileen L Zurbriggen, Ella Ben Hagai, and Gabrielle Leon. Negotiating privacy and intimacy on social media: Review and recommendations. Translational Issues in Psychological Science, 2(3):248, 2016. 
Appendix A

Recruitment Materials 


\section{Part 1: Email to the Administrative Staff}

Hello,

I am a Masters student at Carleton University, working under the supervision of Dr. Sonia Chiasson.

We are looking for volunteers for a research study that will explore the privacy attitudes and behaviours of adolescents with autism spectrum disorder on social media. The results of this study will be used to develop accessible privacy tools and educational programs that will people with autism spectrum disorder to protect their privacy online.

Attached are the study's consent form and recruitment text, which contain more specific details about the research. We would greatly appreciate it if you could share our study information with your members. Please let me know if you would be able to do that.

We would also be delighted to share our findings with you and your organization. We will share a summary with you when the study is completed. The summary will provide tips for adolescents with autism, their parents and anybody who support them to protect their personal information online.

If you would like more information about the study, please feel free to email me at: jessica.rocheleau@carleton.ca.

Please note that the research has been cleared by the Carleton University Research Ethics Board (CUREB-B), REB clearance \#108895.

Thank you!

Jessica Rocheleau

Master's Student

Carleton University 


\section{Part 2: Recruitment Email to Members}

Hello,

Researchers at Carleton University are looking for volunteers for a research study. Each participant will receive $\$ 30$ in cash for their time.

The study will explore how adolescents with autism spectrum disorder protect their privacy on social media. Participants will complete a 1-hour interview about their social media use and their reactions to different online social situations.

The researchers are looking for participants who meet the following criteria:

$\checkmark$ Adolescents aged $13-17$ years old with autism spectrum disorder

$\checkmark$ A regular user of Twitter, Facebook or Instagram

$\checkmark$ Able to understand English

$\checkmark$ Comfortable participating in an interview with or without support

If you are interested in participating, please email the lead researcher, Jessica Rocheleau: jessica.rocheleau@carleton.ca.

Note that this research has been cleared by the Carleton University Research Ethics Board (CUREB-B), REB clearance \#108895.

Thank you! 


\section{Part 1: Message to the Moderator}

Hello,

I am a Masters student at Carleton University, working under the supervision of Dr. Sonia Chiasson.

We are looking for volunteers for a research study that will explore the privacy attitudes and behaviours of adolescents with autism spectrum disorder on social media. The results of this study will be used to develop accessible privacy tools and educational programs that will help people with autism spectrum disorder to protect their privacy online.

Would you be willing to share our study information (attached: social media recruitment text) on your social media page? We would really appreciate it!

We would also be delighted to share our findings with you and your organization. We will share a summary with you when the study is completed. The summary will provide tips for adolescents with autism, their parents and anybody who support them to protect their personal information online.

If you would like more information about the study, please feel free to send me a message or email at: jessica.rocheleau@carleton.ca.

Please note that the research has been cleared by the Carleton University Research Ethics Board (CUREB-B), REB clearance \#108895.

Thank you! 


\section{Part 2: Social Media Recruitment Text in Autism Groups}

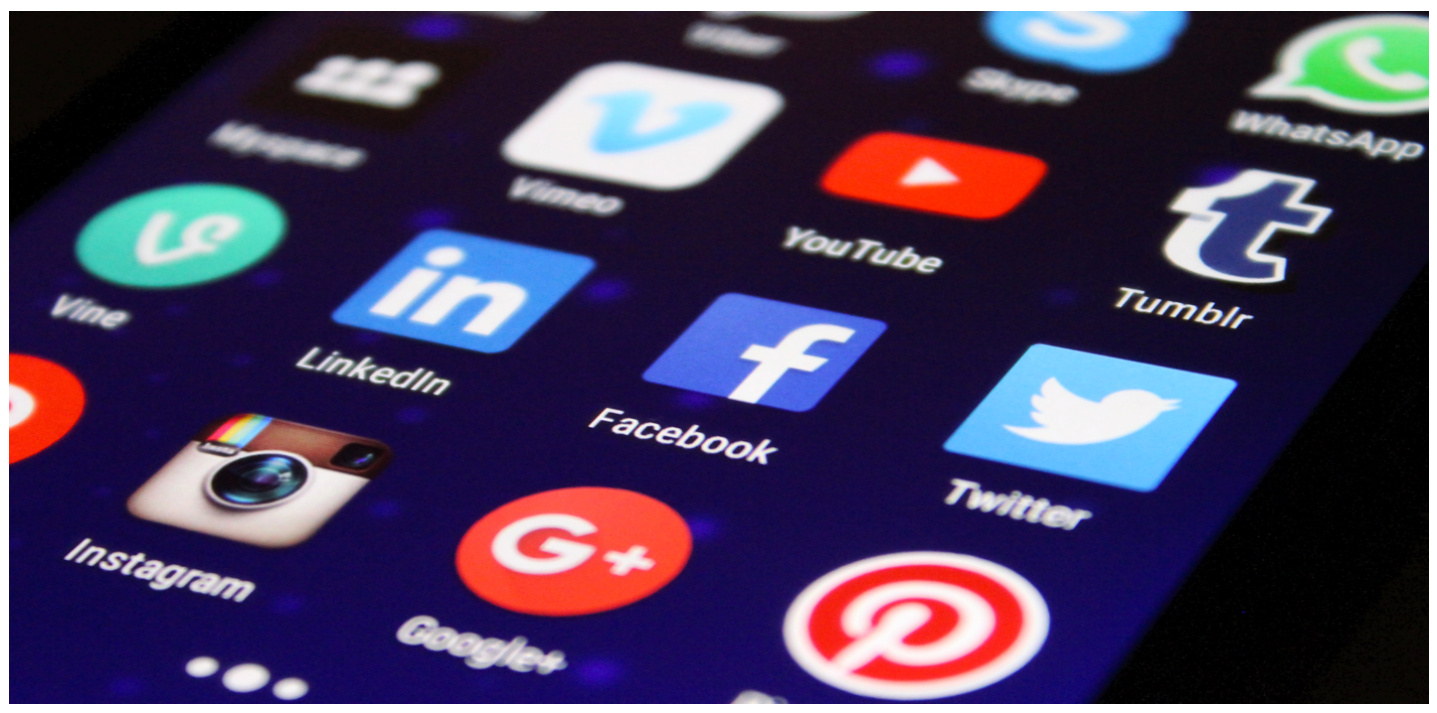

\section{Looking for Volunteers!}

Researchers at Carleton University are looking for volunteers for a research study. Each participant will receive $\$ 30$ in cash for their time.

The study will explore how adolescents with autism spectrum disorder protect their privacy on social media. Participants will complete a 1-hour interview about their social media use and their reactions to different online social situations.

The researchers are looking for participants who meet the following criteria:

$\checkmark$ Adolescents aged $13-17$ years old with autism spectrum disorder

$\checkmark$ A regular user of Twitter, Facebook or Instagram

$\checkmark$ Able to understand English

$\checkmark$ Comfortable participating in an interview with or without support

If you are interested in participating, please contact the lead researcher, Jessica Rocheleau, via email: jessica.rocheleau@carleton.ca. Please feel free to share this post with others.

Please note that this research has been cleared by the Carleton University Research Ethics Board (CUREB-B), REB clearance \#108895. 


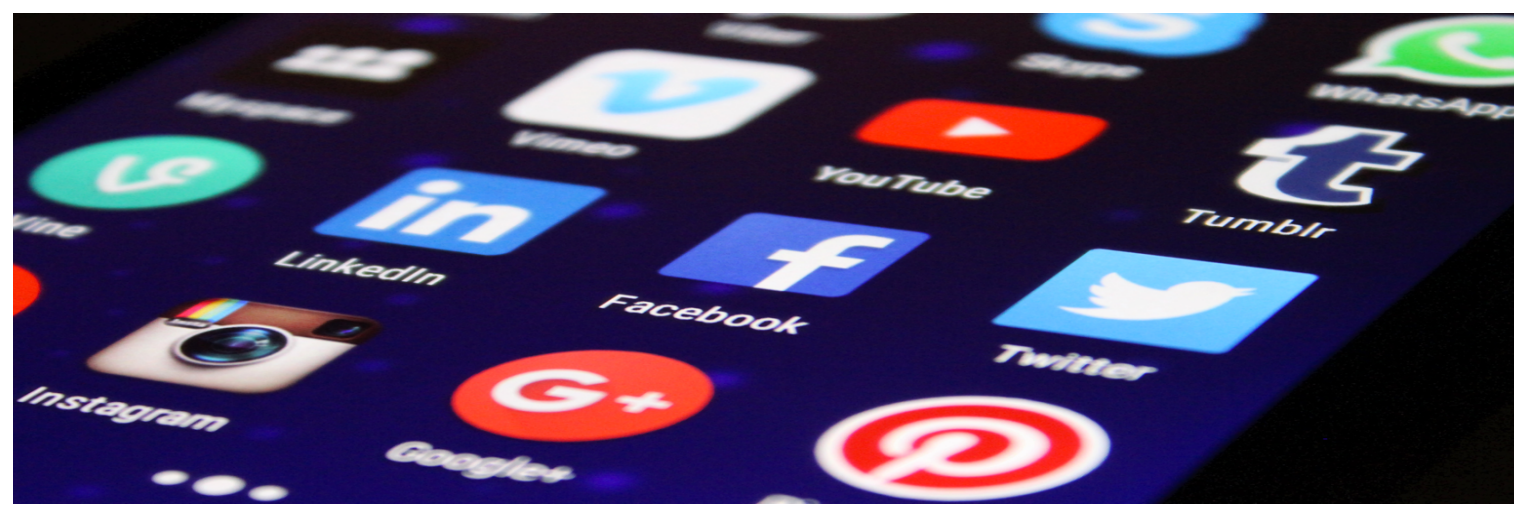

\section{Looking for Research Study Volunteers!}

We are looking for volunteers for a research study exploring how adolescents with or without autism spectrum disorder protect their privacy on social media.

Participants will complete a 1-hour interview about their social media use and their reactions to different online social situations.

Eligibility:

$\checkmark$ Adolescents aged $13-17$ years old with or without autism spectrum disorder

$\checkmark$ A regular user of Twitter, Facebook or Instagram

$\checkmark$ Able to understand English

$\checkmark$ Comfortable participating in an interview with or without support

Compensation: $\$ 30$ in cash

If you are interested in participating, please contact Jessica Rocheleau at: jessica.rocheleau@carleton.ca.

This research has been cleared by the Carleton University Research Ethics Board (CUREB-B), REB clearance \# 108895.

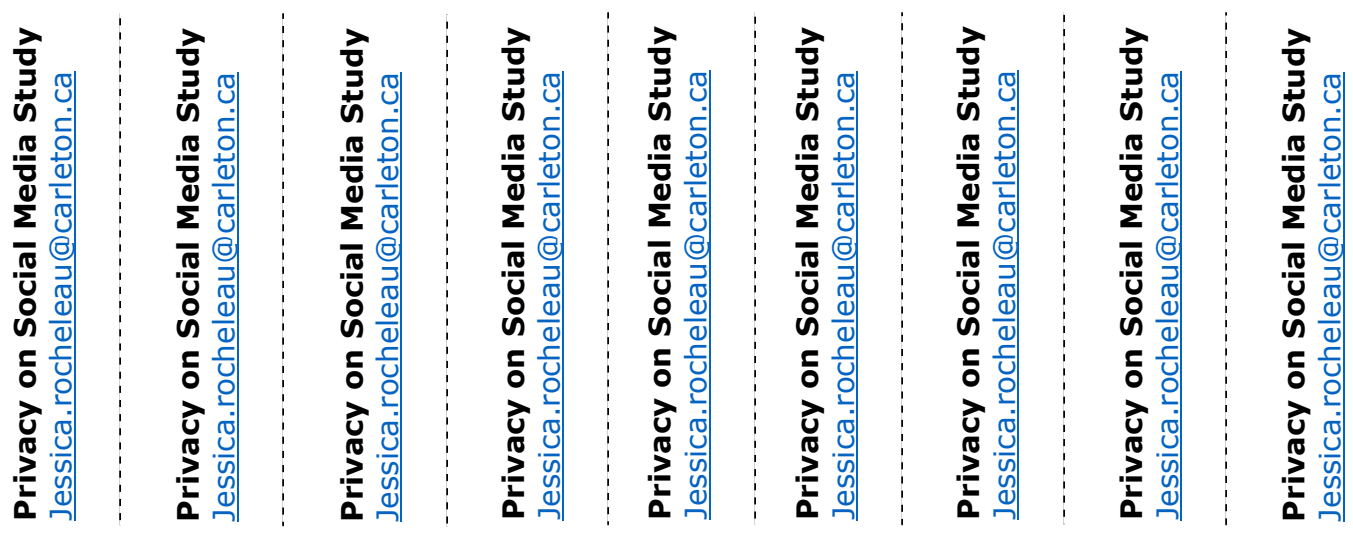


Appendix B

Consent Form 


\section{Carleton \\ U N I V E R S I T Y \\ Canada's Capital University}

\section{Parental Consent Form for Participation in Research}

Title: Investigating the Privacy Attitudes and Behaviours of Adolescents with or without Autism Spectrum Disorder on Social Networking Sites

CUREB-B clearance \#: 108895

Date of ethics clearance: $2018 / 06 / 04$

Ethics Clearance for the Collection of Data Expires: 2019/06/30

\section{Introduction}

The purpose of this form is to provide you (as the parent/legal guardian of a prospective research study participant) with the information that may affect your decision to give consent for your daughter/son to participate in this research study. The researcher for this study, Jessica Rocheleau, is a Masters student in Human-Computer Interaction. She is working under the supervision of Dr. Sonia Chiasson in the School of Computer Science. Please read the information below and ask any questions you might have before making a decision. If you decide to let your daughter/son be involved in this study, this form will be used to record your permission.

\section{Purpose of the Study}

If you agree, your daughter/son will participate in a research study investigating the privacy attitudes and behaviours of adolescents with or without autism on social networking sites (e.g., Facebook, Twitter, Instagram). The results of this study will be used to develop cognitively accessible privacy tools and educational programs that will help users to protect their privacy during online social activities.

What is my daughter/son going to be asked to do? 
Your daughter/son will be asked to complete a 1-hour interview in-person or via Skype. During the interview, your daughter/son will watch short video clips describing different online social situations, and answer some follow-up questions about their privacy-related reactions to the scenarios. None of the videos or social situations will contain any explicit/ adult content. They will also be asked about their own social media use. Please note that your daughter/son is not being tested; we are only interested in their privacy attitudes and behaviours on social networking sites. This research study will be audio-recorded for the purposes of transcription and analysis.

There are no foreseeable risks in participating in this study. Your daughter/son will not be asked to disclose any personally identifiable information. If such information is voluntarily disclosed, it will be discarded when transcribing the interview to text.

\section{Eligibility}

In order to participate in this study, your daughter/son must:

- either have a diagnosis of autism spectrum disorder or not,

- be between the ages of 13 to 17 years,

- be able to understand English, and

- be able to participate in an interview with or without support.

\section{Compensation}

As a token of appreciation, your daughter/son will receive $\$ 30$ in cash for participating in-person, or $\$ 30$ via e-transfer for participating via Skype. We will also reimburse parking costs (if applicable) at the end of the session.

\section{Does my daughter/son have to participate?}

No, your daughter/son's participation in this study is voluntary. In addition to your consent, your daughter/son must also agree to participate in the study. Your daughter/son may decline to participate or to withdraw from participation at any time during the session.

If your daughter/son does not want to participate, they will not be included in the study and there will be no penalty. If your child begins the study and then chooses to withdraw, your child will still receive the $\$ 30$ cash compensation. If your child withdraws, their data will be deleted.

\section{How will my daughter/son's privacy and confidentiality be} protected? 
Your daughter/son's privacy and the confidentiality will be protected. We encourage you to be nearby but not directly sitting with your daughter/son during the session, unless they need help with communication. All responses will be kept anonymous. All research data will be stored and retained on password protected computers or storage devices of the researchers for use in future research. The consent form and notes will be kept in a locked cabinet. Access will be restricted only to the research team. Once the research is completed, audio recordings will be securely destroyed.

If your son/daughter participates through Skype, their data will be stored and protected by Skype servers located in the Unites States, but may be disclosed via a court order or data breach.

\section{What if I do not want my daughter/son to be audio-recorded?}

The session will be audio-recorded to help us accurately note your son/daughter's answers to the interview questions and more easily talk to your son/daughter. The recordings will be deleted after we have transcribed their responses. If you choose to not have your daughter/son audiorecorded, they may still participate in the study. The researcher will take notes of what they said during the study.

The ethics protocol for this project was reviewed by the Carleton University Research Ethics Board, which provided clearance to carry out the research. If you have any ethical concerns with the study, please contact Dr. Andy Adler, Chair, Carleton University Research Ethics Board-B (by phone at 613520-2600 ext. 4085 or via email at ethics@carleton.ca).

Researcher contact information: Jessica Rocheleau, Master's Student Carleton University Email: jessica.rocheleau@cmail.carleton.ca

I, daughter/son, School of Computer Science

Supervisor contact information: Dr. Sonia Chiasson School of Computer Science Carleton University

Tel: (613) 520-2600 ext, 1656 Email: chiasson@scs.carleton.ca give permission for my to participate in this study exploring privacy attitudes and behaviours on social networking sites.

Do you consent to your son/daughter to be audio-recorded for the purpose of transcription? Yes No 
Signature of parent/legal guardian

Signature of researcher
Date

Date

We would also be delighted to share our findings with you. The findings of our study will be published on our lab's website:

http://chorus.scs.carleton.ca. If you would like to receive an executive summary of the research, please provide your email address

Email Address (optional) 


\title{
Appendix C
}

\author{
Interview Guide
}




\section{Interview Guide}

\section{Introduction and Assent}

$\mathrm{Hi}$, my name is Jessica. I am a student at Carleton University. Today, we are going to talk about privacy on [name of social networking site]. During the session, we will look at an [name of social networking site] user's profile and watch some video messages describing different online social situations. I will ask you to give some advice about what the person should do in each situation. I will also ask you about your own experiences using [name of social networking site].

Today's session will take about 30 minutes to complete. I will record your voice so that I remember your comments. If you don't want me to record your voice, please let me know.

The information you will share with me will be safely locked up. No one will hear what you said except for me and my supervisor. Just remember, I am not testing you-I just want to hear your thoughts and opinions.

You have the choice to do this study or not; it is entirely up to you. You can say okay now and change your mind later. All you have to do is tell me that you want to stop. It really is okay to say stop.

To thank you for your help, I will give you $\$ 30$ cash. You can keep the money even if you decide to stop the study.

Do you have any questions? Remember that you can ask questions at any time.

Do you agree to participate in this study?

Do you agree to be audio-recorded?

\section{Scenarios}

Okay, great! So, here is the scenario for this part of the session: An [name of social networking site] user named Allie is looking for advice about how to protect her privacy on the site. This is her account. Allie has left a few video messages with her questions for us to watch and discuss. 
This part of the session will go as follows: I will play the video messages one at a time, asking you a few questions after each one. Feel free to go through Allie's profile if that would help you to answer the question.

Understand?

Great! Here is the first scenario:

\section{Scenario 1 (Dog Photo)}

Video dialogue: Hey! Oh my gosh, guess what? My parents just got me a new puppy. See? (shows picture of dog) I'm so excited. I really want to share this news. Who should I share this with?

1) Who should she share the picture with?

2) Should she share the picture on [name of social networking site]? (Would you?)

3) Who can see Allie's pictures? How do you know?

4) Do you post pictures of yourself on [name of social networking site]?

a. If yes:

- Who do you want to see your pictures?

- Is there anyone you would not like to see your pictures?

- If yes: Do you do anything to make sure that they can't see your pictures?

a. If yes: What do you do?

b. If no: Why not?

\section{Scenario 2 (Stranger)}

Video dialogue: Hi again! So, I just got this notification on my [name of social networking site] feed. It says that someone named Josh Forrester is now following my updates. I've never met this guy before in real life. I took a look at his profile page. It looks like we have a lot in common. But I don't know if I should let him follow me. What should I do?

1) What should Allie do? Is that what you would do in this situation? (If no: What would you do?) 
2) Does it matter that she doesn't know the person in real life? Why or why not?

3) Do you have any followers on [name of social networking site] who you do not know in real life?

a. If yes:

- Do you consider them to be your friends?

- Do you talk to them, through comments or direct messages?

- If yes: What kinds of things do you talk about?

b. If no: Why not?

\section{Scenario 3 (Embarrassing Photo)}

Video dialogue: Hey! So, I was scrolling through my feed and noticed that my friend posted a really embarrassing picture of me. I don't want this picture of me online, but I don't know what to do. What do you think I should do?

1) What should Allie do? Is that what you would do? (If no: What would you do?)

2) What difference does it make if others see the picture?

3) Who can see the picture? How do you know?

4) Are there any pictures of you on [name of social networking site] that you wish were not online?

a. If yes:

- Who posted them?

- Why are they still online?

- Is there anything that you can do to take the pictures off of the site?

\section{Scenario 4 (Private Account)}


Video dialogue: Hi again! So, my friend told me that there's a way to make my account private so that only my friends can see my posts. I don't know how to set my account to private. Do you?

1) Do you know how to make her account private?

a. If yes:

- Can you show me how to do it?

- Who taught you how to do that?

2) Is your [name of social networking site] profile set to private? (If no: Why not?)

\section{Scenario 5 (Anything Else?)}

Video dialogue: Hey! Thanks for all your help. This is my last question. So, I'm trying to set up my profile to make sure that only the right people can see my information. Is there anything else that you do to keep your information safe?

1) Is there anything else that you do to keep your information safe?

\section{Post-Scenario Interview}

\section{Social Media Use}

1) When do you use [name of social networking site]? (How often do you use it?)

2) What do you do on [name of social networking site]?

3) What personal information do you post on [name of social networking site]? (e.g., full name, location, birthday/age, email address, address, phone number)

4) Does anyone help you to use [name of social networking site]?

a. If yes:

- Who? 
- What do they help you to do?

5) Do you talk to people on [name of social networking site]? (If yes: Who do you talk to?)

6) Apart from [name of social networking site], what social networking sites do you use?

\section{Privacy}

1) What does "privacy" mean to you?

2) What does "online privacy" mean to you?

3) Who taught you about online privacy? What kinds of things did they teach you?

4) Do you wish that you knew more about online privacy, or are you happy with how much you know?

\section{Demographic Questions}

1) What is your age?

2) Are you a boy or a girl?

3) What devices do you use to go online?

4) Do you have Internet at home? (If no: Where do you access the Internet?)

\section{Debriefing Script}

Thank you very much for participating! The goal of this research was to better understand how teenagers, like you, protect their privacy on social networking sites. Your thoughts, comments and opinions are very valuable to my research.

Before we wrap up, do you have any questions for me? 